\title{
Tracking the Footprints of Spin Fluctuations: A MultiMethod, MultiMessenger Study of the Two-Dimensional Hubbard Model
}

Thomas Schäfer®, ${ }^{1,2,3,{ }^{*}}$ Nils Wentzell $\odot,{ }^{4}$ Fedor Šimkovic IV, ${ }^{1,2}$ Yuan-Yao He, ${ }^{4,5}$ Cornelia Hille $\odot,{ }^{6}$ Marcel Klett, ${ }^{6,3}$ Christian J. Eckhardt $\odot,{ }^{7,8}$ Behnam Arzhang, ${ }^{9}$ Viktor Harkov $\odot,{ }^{10,11}$ Francois-Marie Le Régent $\odot,{ }^{2}$ Alfred Kirsch, ${ }^{2}$ Yan Wang, ${ }^{12}$ Aaram J. Kim $\odot,{ }^{13}$ Evgeny Kozik $\odot,{ }^{13}$ Evgeny A. Stepanov $\odot,{ }^{10}$ Anna Kauch $\odot,{ }^{7}$ Sabine Andergassen $\odot,{ }^{6}$ Philipp Hansmann $\odot,{ }^{14,15}$ Daniel Rohe $\odot,{ }^{16}$ Yuri M. Vilk, ${ }^{12}$ James P. F. LeBlanc $\odot,{ }^{9}$ Shiwei Zhang $\odot,{ }^{4,5}$ A.-M. S. Tremblay $\odot^{12}{ }^{12}$ Michel Ferrero $\oplus^{1,2}$ Olivier Parcollet ${ }^{4,17}$ and Antoine Georges $\oplus^{1,2,4,18}$

${ }^{1}$ Collège de France, 11 place Marcelin Berthelot, 75005 Paris, France

${ }^{2}$ CPHT, CNRS, École Polytechnique, Institut Polytechnique de Paris, Route de Saclay, 91128 Palaiseau, France

${ }^{3}$ Max-Planck-Institut für Festkörperforschung, Heisenbergstraße 1, 70569 Stuttgart, Germany

${ }^{4}$ Center for Computational Quantum Physics, Flatiron Institute, 162 Fifth Avenue, New York, New York 10010, USA

${ }^{5}$ Department of Physics, College of William and Mary, Williamsburg, Virginia 23187, USA

${ }^{6}$ Institut für Theoretische Physik and Center for Quantum Science,

Universität Tübingen, Auf der Morgenstelle 14, 72076 Tübingen, Germany

${ }^{7}$ Institute of Solid State Physics, TU Wien, A-1040 Vienna, Austria

${ }^{8}$ Institut für Theoretische Festkörperphysik, RWTH Aachen University, 52074 Aachen, Germany

${ }^{9}$ Department of Physics and Physical Oceanography, Memorial University of Newfoundland, St John's, Newfoundland and Labrador, Canada, A1B $3 X 7$

${ }^{10}$ I. Institute of Theoretical Physics, Department of Physics, University of Hamburg, Jungiusstrasse 9, 20355 Hamburg, Germany

${ }^{11}$ European X-Ray Free-Electron Laser Facility, Holzkoppel 4, 22869 Schenefeld, Germany

${ }^{12}$ Départment de Physique, Institut Quantique and RQMP, Université de Sherbrooke, Sherbrooke, Québec, Canada JIK 2RI

${ }^{13}$ Department of Physics, King's College London, Strand, London WC2R 2LS, United Kingdom

${ }^{14}$ Max-Planck-Institut für Chemische Physik fester Stoffe, Nöthnitzerstraße 40, 01187 Dresden, Germany

${ }^{15}$ Department of Physics, University of Erlangen-Nuremberg, 91058 Erlangen, Germany

${ }^{16}$ Forschungszentrum Juelich GmbH, Juelich Supercomputing Centre (JSC), SimLab Quantum Materials, Juelich, Germany

${ }^{17}$ Université Paris-Saclay, CNRS, CEA, Institut de Physique Théorique, 91191, Gif-sur-Yvette, France

${ }^{18}$ DQMP, Université de Genève, 24 Quai Ernest Ansermet, CH-1211 Genève, Switzerland

(Received 18 June 2020; revised 2 November 2020; accepted 21 December 2020; published 23 March 2021)

The Hubbard model represents the fundamental model for interacting quantum systems and electronic correlations. Using the two-dimensional half-filled Hubbard model at weak coupling as a testing ground, we perform a comparative study of a comprehensive set of state-of-the-art quantum many-body methods. Upon cooling into its insulating antiferromagnetic ground state, the model hosts a rich sequence of distinct physical regimes with crossovers between a high-temperature incoherent regime, an intermediate-temperature metallic regime, and a low-temperature insulating regime with a pseudogap created by antiferromagnetic fluctuations. We assess the ability of each method to properly address these physical regimes and crossovers through the computation of several observables probing both quasiparticle properties and magnetic correlations, with two numerically exact methods (diagrammatic and determinantal quantum Monte Carlo methods) serving as a benchmark. By combining computational results and analytical insights, we elucidate the nature and role of spin fluctuations in each of these regimes. Based on this analysis, we explain how quasiparticles can coexist with increasingly longrange antiferromagnetic correlations and why dynamical mean-field theory is found to provide a remarkably accurate approximation of local quantities in the metallic regime. We also critically

\footnotetext{
*t.schaefer@fkf.mpg.de
}

Published by the American Physical Society under the terms of the Creative Commons Attribution 4.0 International license. Further distribution of this work must maintain attribution to the author(s) and the published article's title, journal citation, and DOI. 
discuss whether imaginary-time methods are able to capture the non-Fermi-liquid singularities of this fully nested system.

DOI: 10.1103/PhysRevX.11.011058

Subject Areas: Computational Physics

Condensed Matter Physics

Strongly Correlated Materials

\section{INTRODUCTION}

\section{A. Purpose of this article}

For interacting quantum systems, the Hubbard model [1-4] has a status similar to the Ising model for classical phase transitions and magnetism. It is the simplest possible model that can be considered, and it nonetheless captures essential aspects of the physical phenomena of interest. In relation to materials with strong electronic correlations, the Hubbard model in its simplest form (especially with a single band) is, at best, an approximation to reality. However, experimental progress in the field of cold atomic gases in optical lattices now yields rather accurate physical realizations of this simple model in the laboratory [5-7].

In contrast to the Ising model, however, our current understanding of the Hubbard model is still lacunary. A thorough understanding can be reached in the limiting cases of one dimension [8] and infinite dimensions (infinite lattice connectivity) [9-11], thanks to efficient analytical and computational methods available in these limits. In contrast, the two-dimensional case relevant to both cuprate superconductors [12-15] and cold atomic gases [5-7] still holds many open questions, both in relation to its phase diagram as a function of interaction, particle density, and temperature, and regarding the nature of excited states as well as response functions and transport properties. There is a broad consensus in the community that progress on these outstanding issues is essential, even if addressed through the deceptively simple-looking Hubbard model.

In recent years, a number of computational methods have been developed to this end. In this context, it is of crucial importance to interrogate these methods regarding their respective ability to address regimes of physical interest. Furthermore, increasing emphasis is being placed on establishing definite results with controlled computational methods, which can then serve as benchmarks [16-18] for approximate, often more flexible and computationally efficient methods.

Here, we focus on a regime of the Hubbard model that is simple at first sight but, as we shall see, deceptively so: small interaction values (often referred to as "weak coupling") and half-filling on the square lattice with nearestneighbor hopping. The main purpose of this article is to assess the ability of state-of-the-art computational methods to address the finite-temperature physics of the model in this regime. We provide an extensive comparison between basically all methods currently available for this purpose, with two distinct Monte Carlo methods serving as reference benchmarks. Despite the apparent simplicity of this regime, we show that all methods face rather severe limitations, especially regarding the lowest temperature that can currently be reached. Our study also interrogates the model through a set of different physical observables, spanning thermodynamic properties and single-particle correlation functions (the Green function and associated self-energy) as well as two-particle correlations (the spin correlation function and correlation length). Because of this wide spectrum of both methods and observables, we borrow terminology from the astrophysics community in designating our work as a "multimethod, multimessenger study" [19].

Despite the deceptive simplicity of the two-dimensional Hubbard model in this parameter regime, the physics is quite rich and nontrivial. As is well established, the ground state is an antiferromagnetic insulator that can be qualitatively understood using Slater's classic description [20]. However, finite-temperature properties display a rich sequence of interesting crossovers between physically distinct regimes as the system is cooled down towards its antiferromagnetic insulating ground state. Two key features make these finite-temperature properties and crossovers nontrivial: (i) the fact that, despite perfect nesting, fluctuations destroy antiferromagnetic long-range order at any nonzero temperature-while the correlation length is exponentially large (Mermin-Wagner theorem [21,22]) and (ii) the van Hove singularity present at the "antinodal" points of the Fermi surface, which further suppresses the coherence of single-particle excitations near these points. Furthermore, the perfect nesting of the Fermi surface in combination with two-dimensionality leads to a departure from Fermi-liquid behavior in the metallic regime.

Another important goal of this article is therefore to discuss and characterize these different physical regimes and crossovers in detail, with a particular focus on assessing the ability of the different methods to capture their physics properly. By combining computational results and analytical insights, we elucidate, in particular, the role and nature of spin fluctuations in the different regimes. As we shall see, this analysis reveals some unexpected features of the metallic regime, which are likely to have broader implications for materials with electronic correlations. Furthermore, we discuss whether the imaginary-time computational methods considered in this article are able to probe the subtle non-Fermi-liquid singularities of the metallic state caused by the perfect nesting of the Fermi 
surface. This rich physics thus makes this regime of the Hubbard model a perfect opportunity for systematic benchmarks, as well as a useful testing ground for future work on more complex regimes of parameters as well as real materials.

\section{B. Overview of the methods assessed}

We categorize the algorithms considered in this article into the following groups:

(i) Benchmark methods. We consider two very different Monte Carlo methods. The first one is the determinantal quantum Monte Carlo method (DQMC, Ref. [23]), and the other is the diagrammatic Monte Carlo method (later referred to as DiagMC) [24] [in its recent connected determinant implementation (CDet) for connected one-particle reducible quantities [25] and $\Sigma$ DDMC for one-particle irreducible quantities [26,27], respectively]. The reason for their application is twofold: (1) The methods are controlled; hence, in the regime where they can be applied and converged, they are numerically exact. Therefore, they serve as a benchmark for the other approximate methods considered in this article. (2) The regimes where the methods actually break down will be assessed, providing crucial information for their application to more challenging regimes. For both benchmark methods, we show error bars in the figures (which may, however, be smaller than the respective symbol sizes). All data points in this paper obtained by these methods are numerically exact.

(ii) Mean-field methods. In Sec. III, we discuss dynamical mean-field theory (DMFT, Ref. [28]) as a reference point, beyond which spatial fluctuations must be included to properly address the twodimensional model. In that section, we also briefly discuss simple, static, mean-field theory (MFT). As we shall see, DMFT provides a good starting point for our study and, remarkably, yields an accurate approximation of local observables through most of the metallic regime.

(iii) Cluster extensions of DMFT. The dynamical cluster approximation (DCA) and cellular DMFT (CDMFT) provide one possible route to systematically include spatial correlations within the DMFT framework, beyond the single-site approximation [29-32]. Note that cluster-based methods (like CDMFT, DCA, and also cluster-TRILEX, discussed below) are controlled methods, with the control parameter being the size of the cluster. However, in some regimes shown in this paper (low temperatures), these algorithms cannot be converged as a function of cluster size (for reasons explained in the Appendixes D 5 and D 6).

(iv) Vertex-based extensions of DMFT. Another route for including spatial correlations beyond singlesite DMFT relies on higher-order Green functions (vertex functions). In the main text, we present results from the dynamical vertex approximation (DГA, ladder version), the triply irreducible local expansion (TRILEX) in various flavors, the dual fermion (DF, ladder version), and the dual boson (DB, single-shot) approach [33].

(v) Other approaches. In this category, we show results from the two-particle self-consistent approach (TPSC, TPSC+, Refs. [34-36]), the functional renormalization group (fRG [37]; here, considered up to one loop with Katanin substitution), and the parquet approximation (PA [38,39]).

This list covers the vast majority of currently available computational methods able to address finite-temperature properties. One notable exception is the minimally entangled, typical thermal state method (METTS) and related approaches, which combine tensor network representations and stochastic sampling [40-43]. A systematic exploration of this method as applied to the Hubbard model is currently being actively pursued by several groups, and comparisons with the present methods will have to be performed in future work [44].

The basic principles of each of these methods, useful references for further reading, and results from slightly differing implementations of the respective methods and algorithms are summarized in Appendix D. Throughout the paper, we consider the interaction value of $U=2 t$. Let us stress from the outset that, despite this rather moderate interaction value, each of these methods encounters limitations in their regime of applicability. These limitations stem from either (i) the approximation performed or (ii) algorithmic obstacles.

We find that the lowest reachable temperature for the DiagMC algorithm is $T_{\text {min }}^{\text {DiagC }} \approx 0.06 t$. In this case, reaching lower temperatures is hindered by the difficulty in summing the perturbative series. Interestingly, we find that the limitation of the DQMC algorithm is similar, $T_{\min }^{\mathrm{DQMC}} \approx 0.06 t$. In that case, the limitations originate from the exponentially growing correlation length, which would require the simulation of prohibitively large systems at lower $T$. DMFT, in contrast, can be converged to very low temperatures and also at $T=0$. Self-consistent methods (e.g., TRILEX) suffer from convergence problems at low $T$, whereas calculations involving a "single-shot" correction beyond DMFT without self-consistency such as DГA, DB, or DF can be performed as long as the correlation length can be accurately resolved (DГA and $\mathrm{DB}$ ) or, for $\mathrm{DF}$, as long as the starting point-paramagnetically restricted DMFT - remains reasonably accurate. The finite momentum grid limits the application of fRG and PA and, to a lesser degree, TPSC and TPSC+. An intrinsic limitation of TPSC occurs in the renormalized classical regime (see Appendix D 11), leading to a rather severe overestimation of the onset temperature of the pseudogap. TPSC+ has been proposed as a solution: In the present paper, the first 
application of TPSC+ is actually presented, but its applicability has yet to be explored more widely. Obvious limitations of quantum cluster theories are the cluster sizes that they can reach, which have to be compared to the correlation length - a very demanding criterion in the present case, as will be shown later.

\section{Definition of the model, the role of the van Hove singularity, and nesting}

We consider the single-band Hubbard model defined by the following Hamiltonian:

$$
H=-t \sum_{\langle i j\rangle, \sigma} c_{i \sigma}^{\dagger} c_{j \sigma}+U \sum_{i} n_{i \uparrow} n_{i \downarrow},
$$

where $t$ is the (nearest-neighbor) hopping amplitude, $\langle i j\rangle$ denotes summation over nearest-neighbor lattice sites, $\sigma \in$ $\{\uparrow, \downarrow\}$ is the electron's spin, $U$ is the strength of the (purely local) Coulomb interaction, and $n_{i \sigma}=c_{i \sigma}^{\dagger} c_{i \sigma}$ is the spinresolved number operator. Throughout the paper, all energies are given in units of $t=1$. Furthermore, we set $\hbar=1$ and $k_{\mathrm{B}}=1$. We consider the case of $U=2$ (usually regarded as weak coupling) at half-filling $n=\left\langle n_{\uparrow}+n_{\downarrow}\right\rangle=1$, corresponding to a chemical potential of $\mu=U / 2=1$ and the simple square lattice, resulting in the following dispersion relation for the electrons (lattice constant $a=1$ ):

$$
\varepsilon_{\mathbf{k}}=-2\left[\cos \left(k_{x}\right)+\cos \left(k_{y}\right)\right]
$$

The particular form of the dispersion and the case of halffilling leads to a very peculiar diamond-shaped Fermi surface, already resulting in an interesting behavior without interactions present: (i) It exhibits a ("perfect") nesting by the momentum vector $\mathbf{Q}=(\pi, \pi)$, which connects every Fermi-surface point to another respective one (see Fig. 1), leading to an enhanced susceptibility at $\mathbf{q}=\mathbf{Q}$; and (ii) the momenta around the (stationary) antinodal Fermi-surface point $\mathbf{k}_{\mathrm{AN}}=(\pi, 0)$ imply a logarithmic divergence in the density of states $\rho_{0}(\varepsilon)$ at the Fermi level (van Hove singularity, Fig. 1), leading to a larger scattering phase space than at the nodal point $\mathbf{k}_{N}=(\pi / 2, \pi / 2)$. Furthermore, because we consider only nearest-neighbor hopping, the diamond-shaped Fermi surface displays perfect nesting by the whole family of wave vectors of the form $\left(q_{x}, \pm q_{x}\right)$, with consequences for the nature of the metallic regime.

\section{Organization of this article}

This article is organized as follows: In Sec. II, we describe the different physical regimes encountered in this model as a function of temperature, using results from our two benchmarks methods (DiagMC and DQMC). In Sec. III, we discuss dynamical mean-field theory, which serves as a starting point for several approximate methods
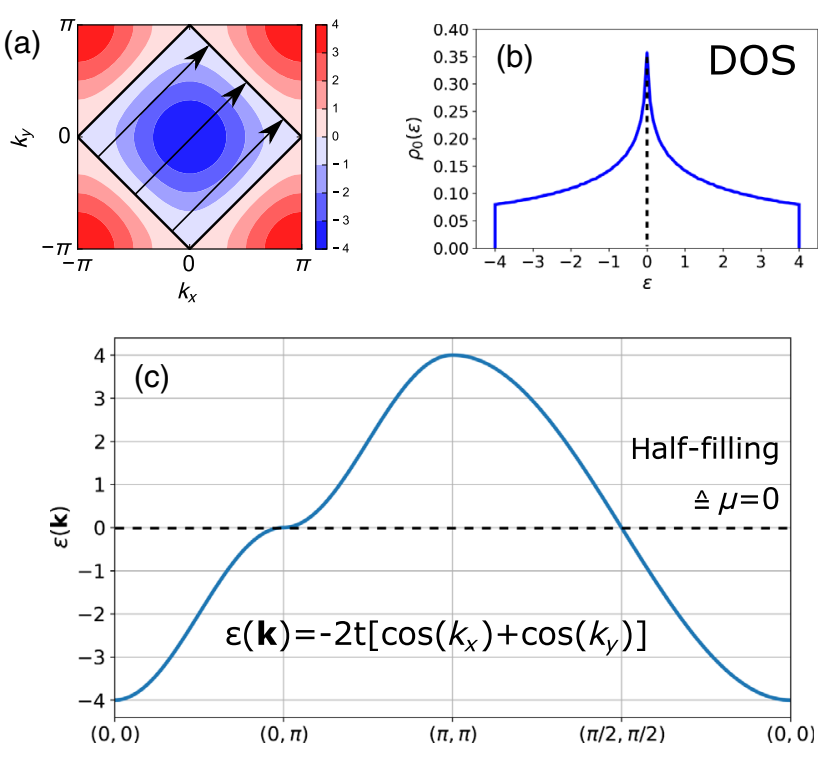

FIG. 1. (a) Momentum distribution of the (noninteracting) dispersion relation of the simple square lattice, Eq. (2), for $t=1$ throughout the first Brillouin zone. The Fermi surface of the half-filled system $(\mu=0)$ is diamond shaped (bold black); the black arrows indicate the nesting vectors, mutually connecting Fermi-surface points. (b) Corresponding (particle-hole symmetric) density of states (DOS) as a function of energy $\rho_{0}(\varepsilon)$. Here, $\varepsilon=0$ corresponds to half-filling. (c) Value of the dispersion relation along a high-symmetry path exhibiting a plateau around $(\pi, 0)$, leading to a vanishing Fermi velocity $v_{\mathrm{F}}$.

considered in this article. In Sec. IV, we discuss the calculation of single-particle properties using all the different methods introduced above. In Sec. V, we discuss the $T$ dependence of the double occupancy and its physical significance. In Sec. VI, we discuss two-particle response functions and the $T$ dependence of the magnetic correlation length. In Sec. VII, we discuss the implications of our computational results for the physics of spin fluctuations in this model. Finally, a discussion and conclusions are provided in Sec. VIII. A number of Appendixes present more technical points as well as details of the different methods. In the Supplemental Material [45] we provide all the numerical data used in the figures of the main text.

\section{QUALITATIVE DESCRIPTION: PHYSICAL REGIMES AND CROSSOVERS}

Before presenting detailed results from a variety of many-body approaches in Secs. III-VI, in this section we sketch the overall physical picture that emerges from this study in Fig. 2 (see also Refs. [47-49]). The left panel indicates, in a schematic manner, the key crossover scales that delimit different physical regions as a function of temperature $T$, for a given value of $U$. Our quantitative study focuses on $U=2$, but the qualitative statements made here are expected to apply throughout the weak- to intermediate-coupling regime (see, e.g., Ref. [50] for a 


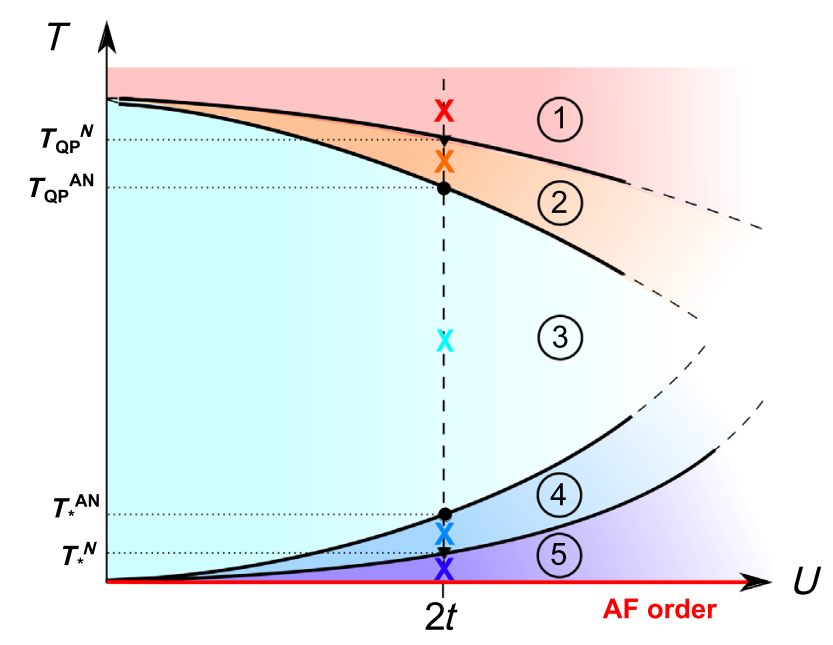

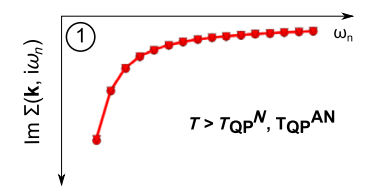
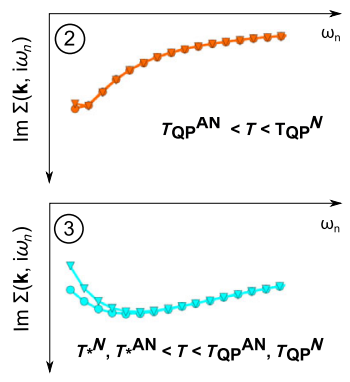

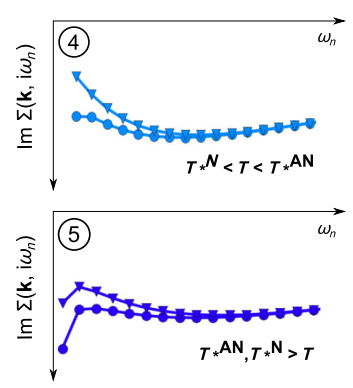

$\bullet A N=(\pi, 0)$
$\nabla=(\pi / 2, \pi / 2)$

FIG. 2. Left panel: schematic phase diagram of the two-dimensional Hubbard model on a simple square lattice in the weak-coupling regime around $U=2 t$. Here, $T_{\mathrm{QP}}^{N}$ and $T_{\mathrm{QP}}^{\mathrm{AN}}$ denote the onset of coherence at the nodal $\mathbf{k}=(\pi / 2, \pi / 2)$ (triangle) and antinodal $\mathbf{k}=(\pi, 0)(\mathrm{dot})$ Brillouin zone points, respectively. The onset of the (pseudo)gap at the antinode and node is denoted by $T_{*}^{\mathrm{AN}}$ and $T_{*}^{N}$, respectively. Right panels: qualitative sketches of the imaginary parts of the self-energy on the Matsubara axis (extracted from DГA calculations) for temperatures corresponding to the colors given in the phase diagram.

study of the evolution to higher couplings). As the system is cooled down from high temperature, we observe several regimes with qualitatively different physical properties (a quantitative criterion for the onset of these scales will be given at the end of the section).

At high temperature, thermal fluctuations prevent the formation of long-lived quasiparticles: This regime can be thought of as an "incoherent soup" of fermions above their degeneracy temperature and is depicted as the red shaded area (1) in Fig. 2. Cooling the system progressively extinguishes these thermal fluctuations, leading to increased coherence in the single-particle spectrum and the appearance of long-lived quasiparticles. Here and below, we use the term "quasiparticle" in a general and somewhat loose sense of a dispersing single-particle excitation with a "long enough" lifetime. For the specific model at hand, because of perfect nesting, the quasiparticles do not obey Landau's Fermi-liquid theory, however: This case is discussed in more detail in Sec. VII C 3. At the node, this quasiparticle coherence scale $T_{\mathrm{QP}}^{N}$ corresponds to the temperature at which the thermal de Broglie wavelength $v_{\mathrm{F}}^{*} /(\pi T)$ along the nodal direction becomes larger than the lattice spacing, with $v_{F}^{*}$ being the effective Fermi velocity renormalized by interactions. The metallic regime is depicted as region (3) (light blue) in Fig. 2.

The crossover scale associated with the passage from region (1) to region (3) is not the same all along the Fermi surface, however. Because of the van Hove singularity stemming from the antinodal points of the Fermi surface such as $(\pi, 0)$ (see Sec. I C), the coherence temperature $T_{\mathrm{OP}}^{N}$ at the nodal point $\mathbf{k}_{N}=(\pi / 2, \pi / 2)$ is higher than the coherence temperature at the antinodal point $T_{\mathrm{QP}}^{\mathrm{AN}}<T_{\mathrm{QP}}^{N}$. This differentiation defines an extended crossover region (2) in which the system is coherent near the nodes but still incoherent near the antinodes (orange shaded area in Fig. 2). Although further lowering the temperature in the metallic regime (3) initially results in freezing out thermal fluctuations and hence in an increase of the quasiparticle lifetime, this does not persist down to the lowest temperature. Indeed, antiferromagnetic correlations develop as $T$ is lowered, with an exponentially growing correlation length, eventually diverging at $T=0$ when the ground state with antiferromagnetic long-range order is reached.

In this low- $T$ regime, long-wavelength antiferromagnetic fluctuations (Slater paramagnons) lead to an enhancement of the quasiparticle scattering rate upon cooling and to the formation of a pseudogap in the single-particle spectrum, which evolves into a sharp gap in the Slater-like insulator at $T=0$ [20]. Once again, the crossover temperature $T_{*}$ corresponding to the suppression of coherence and the opening of the pseudogap is not uniform along the Fermi surface: It is larger at the antinodes where the destruction of coherence occurs first upon cooling and smaller at the nodes: $T_{*}^{N}<T_{*}^{\mathrm{AN}}$. Hence, in the dark-blue shaded area (4) where $T_{*}^{N}<T<T_{*}^{\mathrm{AN}}$, one has a partially (pseudo)gapped Fermi surface. Eventually, all states of the Fermi surface are suppressed by antiferromagnetic fluctuations for $T<T_{*}^{N}$, resulting in a fully open pseudogap everywhere on the Fermi surface (purple shaded area (5)). Let us stress again that long-range antiferromagnetic order and a true gap only set in at $T=T_{\text {Néel }}=0$ as a consequence of the MerminWagner theorem $[21,22]$.

Since all the temperature scales described above correspond to crossovers, an appropriate criterion must be defined to identify and quantify them. These scales mostly refer to the presence or absence of characteristic spectral features in the single-particle properties, and hence, an 
TABLE I. Temperature scales discriminating the regions (1)-(5) of Fig. 2 calculated using various many-body techniques.

\begin{tabular}{|c|c|c|c|c|c|}
\hline Method & $T_{\mathrm{QP}}^{N}$ & $T_{\mathrm{QP}}^{\mathrm{AN}}$ & $T_{*}^{\mathrm{AN}}$ & $T_{*}^{N}$ & $T_{\text {Néel }}$ \\
\hline DiagMC & 0.42 & 0.35 & 0.065 & 0.0625 & 0 \\
\hline DQMC & 0.42 & 0.35 & 0.065 & 0.0625 & 0 \\
\hline MFT & $\infty$ & $\infty$ & 0.2 & 0.2 & 0.2 \\
\hline DMFT & 0.45 & 0.45 & 0.08 & 0.08 & 0.08 \\
\hline $\mathrm{DCA}, N_{c}=128(\mathrm{PM}$ enforced $)$ & 0.42 & 0.35 & $\ldots$ & $\ldots$ & $\ldots$ \\
\hline CDMFT, $N_{c}=64$ (PM enforced) & 0.45 & 0.42 & $\cdots$ & $\cdots$ & see Sec. VI \\
\hline $\mathrm{CDMFT}+\mathrm{CFE}, N_{c}=64(\mathrm{PM}$ enforced $)$ & 0.42 & 0.35 & $\cdots$ & $\cdots$ & see Sec. VI \\
\hline DГА (ladder) & 0.42 & 0.35 & 0.065 & 0.059 & $0[51,53,54]$ \\
\hline DF (ladder) & 0.44 & 0.37 & 0.062 & 0.06 & $0(\cdots)$ \\
\hline DB (single-shot) & 0.42 & 0.35 & $<0.07$ & $<0.07$ & $0(\cdots)$ \\
\hline TRILEX & 0.44 & 0.35 & $<0.055$ & $<0.055$ & $0(\cdots)$ \\
\hline TRILEX, $\Lambda^{2}$ & 0.44 & 0.35 & $<0.055$ & $<0.055$ & $0(\cdots)$ \\
\hline TRILEX, $N_{c}=2$ & 0.44 & 0.35 & $<0.055$ & $<0.055$ & $0(\cdots)$ \\
\hline TRILEX, $N_{c}=4$ & 0.44 & 0.35 & $<0.055$ & $<0.055$ & $0(\cdots)$ \\
\hline TPSC & 0.42 & 0.29 & 0.13 & 0.1 & 0 \\
\hline TPSC+ & 0.44 & 0.37 & 0.07 & $<0.07$ & 0 \\
\hline fRG (one-loop Katanin) & 0.42 & 0.35 & 0.08 & $\cdots[55-59]$ & $>0[55,60-62]$ \\
\hline PA & 0.44 & 0.37 & $<0.05$ & $<0.05$ & $0[60-62]$ \\
\hline
\end{tabular}

obvious observable would be the momentum- and energyresolved spectral function $A(\mathbf{k}, \omega)=-(1 / \pi) \operatorname{Im} G(\mathbf{k}, \omega+$ $i 0^{+}$) and the corresponding self-energy as a function of real frequency $\omega$ (see also Appendix B and Ref. [51]). However, as all the methods considered in the following are formulated on the Matsubara (imaginary) frequency axis, a much more practical criterion can be obtained via the imaginary frequency dependence of the (imaginary part of the) momentum-resolved self-energy $\operatorname{Im} \Sigma\left(\mathbf{k}, i \omega_{n}\right)$, which is consistent with previous work $[47,48,52]$. Representative results for this quantity in the five different regimes discussed above are displayed in the right panel of Fig. 2. At high temperature, the thermal fluctuations lead to a divergent behavior of $\operatorname{Im} \Sigma\left(\mathbf{k}, i \omega_{n}\right)$ at low frequencies. Thus, $T_{\mathrm{QP}}$ can be defined as the temperature where this divergent behavior is eased, i.e., when the slope between the first and second Matsubara frequencies changes sign and becomes negative $[47,48,52]$. In the metallic regime
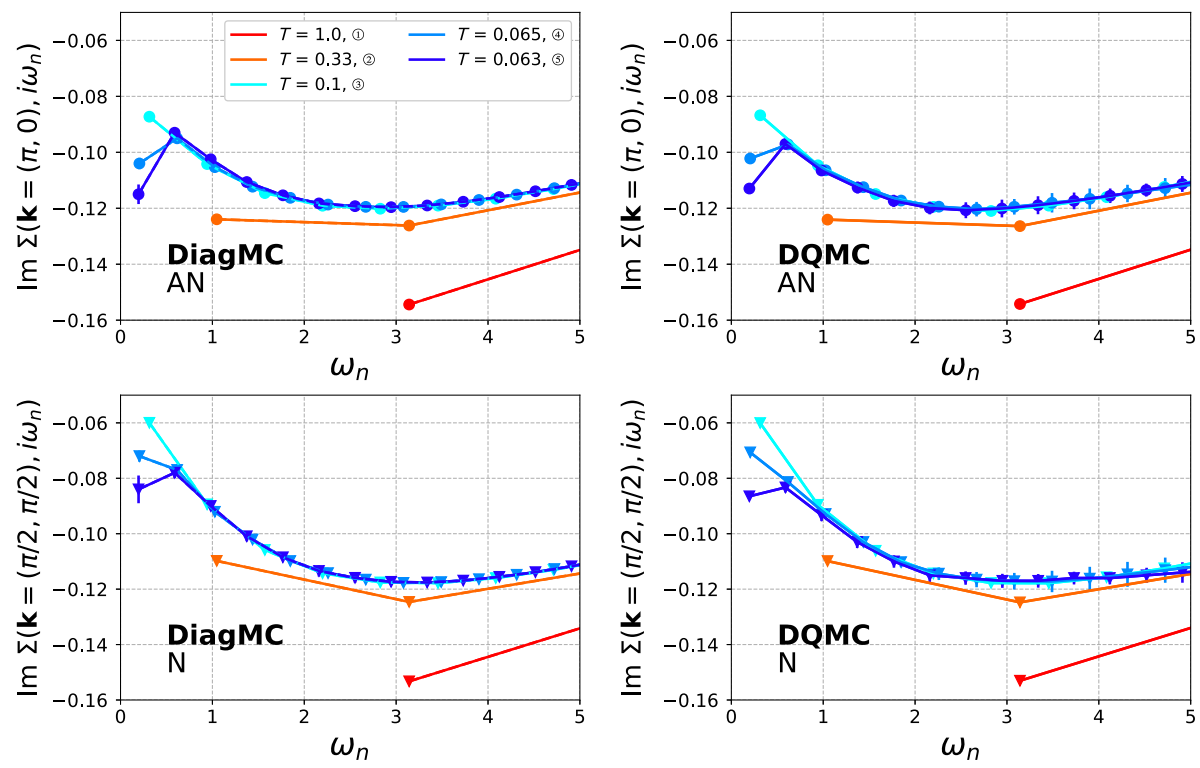

FIG. 3. Imaginary part of the self-energy for the antinode (upper row) and node (lower row) as a function of Matsubara frequencies for the two numerically exact techniques, DiagMC (left panels) and DQMC (right panels). Note that the error bars may be of the order of the marker size. 
(region (3), the behavior of $\operatorname{Im} \Sigma\left(\mathbf{k}, i \omega_{n}\right)$ over the lowest Matsubara frequencies can be approximated by a Taylor series $\operatorname{Im} \Sigma\left(\mathbf{k}, i \omega_{n}\right)=-\gamma_{\mathbf{k}} / Z_{\mathbf{k}}+\omega_{n}\left(1-1 / Z_{\mathbf{k}}\right)+\cdots$. In a standard Fermi liquid, $Z_{\mathbf{k}}$ and $\gamma_{\mathbf{k}}$ are the quasiparticle spectral weight $\left(Z_{\mathbf{k}}<1\right)$ and inverse quasiparticle lifetime, respectively (see Appendix B for details and a critical discussion). Likewise, the onset of insulating or pseudogapped behavior at $T_{*}^{N, \mathrm{AN}}$ is signaled by an additional change of slope, which becomes positive again at lower temperatures. The actual quantitative criterion for extracting the data of Tables I using the quasiparticle weight $Z_{\mathbf{k}}$ is also discussed in Appendix B.

To illustrate the onset of these different regimes at the oneparticle level, we display in Fig. 3 results for the imaginary part of the self-energy on the Matsubara axis calculated with the two numerically exact methods used in this paper, namely, DiagMC (left panels; see Appendix D 1) and DQMC (right panels; for technical details, including finite-size scaling and Trotter extrapolation, see Appendix D 2, and for preceding works, see also Refs. $[47,63,64])$. Both exact methods shown in Fig. 3 exhibit $T_{\mathrm{QP}}$ and $T_{*}$, with a momentum differentiation between the antinode (upper panels) and node (lower panels), with $T_{\mathrm{QP}}^{N} \approx$ 0.42 and $T_{\mathrm{QP}}^{\mathrm{AN}} \approx 0.35$ for the onset of the quasiparticle coherence and $T_{*}^{\mathrm{AN}} \approx 0.065$ and $T_{*}^{N} \approx 0.0625$ for the onset of the insulating behavior (more intermediate temperatures have been calculated for the extraction; see Fig. 19 and Appendix B). DiagMC and DQMC are in agreement within error bars and (for the one-particle quantities) are able to be converged until $T \approx 0.063$. With the aim of giving an overview and for further reference, we summarize, in Table I, the results of each of the different methods investigated below for the crossover temperatures delimiting the five distinct regimes discussed above.

\section{DYNAMICAL MEAN-FIELD REFERENCE POINT AND THE ROLE OF FLUCTUATIONS}

Several methods considered in the following use dynamical mean-field theory (DMFT) as a starting point. Within DMFT, local fluctuations are taken into account (i.e., quantum and thermal fluctuations between the four possible local states on each site), but spatial fluctuations are not. In the two-dimensional model considered here, these fluctuations are strong, and DMFT should be viewed as a zeroth-order approximation, which needs to be extended in order to take these fluctuations into account. DMFT is exact in the formal limit of infinite dimensions or infinite lattice connectivity, in which spatial fluctuations become negligible $[9,10]$.

\section{A. Self-energies and quasiparticle coherence}

Because nonlocal fluctuations are neglected, the selfenergy is approximated within DMFT by a function that is local in real space, i.e., independent of momentum. In Fig. 4, we display the DMFT self-energy for several temperatures and compare it to the benchmark (DiagMC) result for both the antinodal and nodal points (left and central panels, respectively). It is seen that the DMFT approximation is accurate at very high temperatures, where, indeed, correlations are very local (as shown below, the magnetic correlation length is only a couple of lattice spacings down to $T \simeq 0.2$ ). Deviations between the DMFT self-energy and the DiagMC benchmark are already apparent at $T \simeq 0.33$ : At this temperature, these deviations are small at the nodal point but already significant at the antinodal point due to the proximity of the van Hove singularity and the resulting momentum dependence of the self-energy.

However, it is interesting to note that the local component of the self-energy (i.e., on site or, equivalently, momentum integrated) is in good agreement with DMFT down to a much lower temperature. As shown in Fig. 5, the local self-energy obtained from DiagMC is still on top of the DMFT result for temperatures as low as $T=0.1$, close to the DMFT ordering temperature. This result may come as a surprise in view of the fact that the antiferromagnetic correlation length (Sec. VI) is sizable at this temperature, of the order of five lattice spacings. In Sec. VII, we provide an explanation to this observation and further discuss its physical significance.

From the results in Fig. 4 (and more temperature points, not shown), one observes that, within DMFT, the onset of
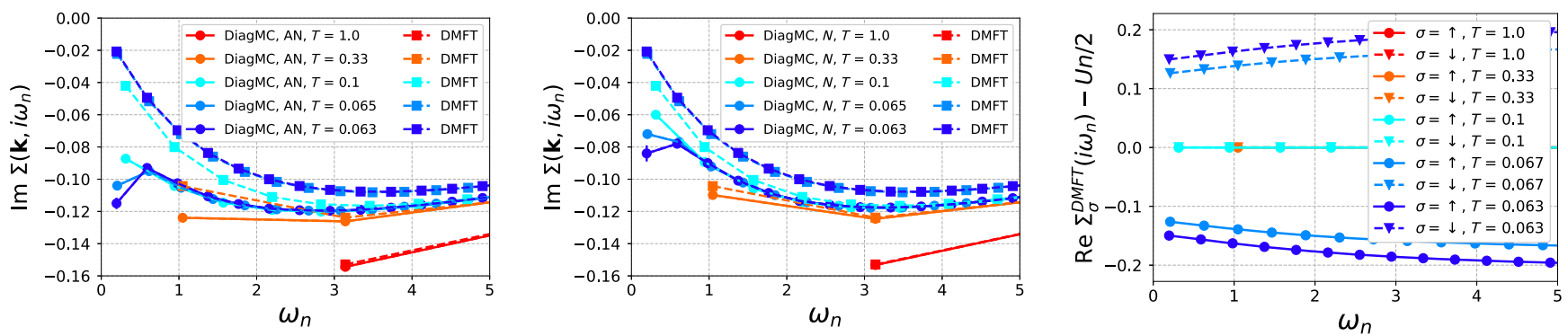

FIG. 4. Left and central panels: imaginary part of the self-energy as a function of frequency at the antinode (left panel) and node (central panel) in DiagMC (circles, solid lines) and the DMFT impurity self-energy (squares, dashed lines) for various temperatures. Right panel: spin-resolved real part of the DMFT self-energy as a function of Matsubara frequency for several temperatures (the Hartree term of $U n / 2=1$ has been subtracted). Note that $T_{\text {Néel }}^{\mathrm{DMFT}} \approx 0.08$, and for $T<T_{\text {Néel }}^{\mathrm{DMFT}}: \Sigma_{\uparrow} \neq \Sigma_{\downarrow}$. 


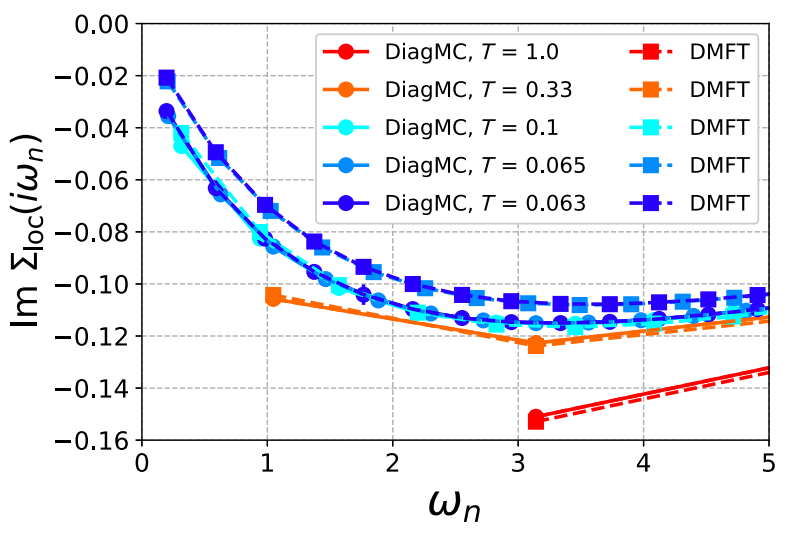

FIG. 5. Imaginary part of the local self-energy calculated by DiagMC compared to DMFT. Note that the two lowest temperatures displayed, at which a discrepancy appears, correspond to the DMFT ordered phase.

quasiparticle coherence associated with the crossover from the high- $T$ incoherent regime into the lower- $T$ metallic regime (regime (1) to regime (2) in Fig. 2) occurs at $T_{\mathrm{QP}}^{\mathrm{DMFT}} \simeq 0.45$. This result is in reasonable agreement with the QP coherence scale at the node from our benchmark calculations $T_{\mathrm{QP}}^{N} \simeq 0.42$ but somewhat higher than the antinodal value $T_{\mathrm{QP}}^{\mathrm{AN}} \simeq 0.35$, again due to the lack of momentum dependence of the self-energy, which is essential to account for nodal-antinodal differentiation. We have calculated the quasiparticle coherence temperature within DMFT as a function of coupling $U$, and we display the result in Fig. 6 (red line in upper panel). As expected, this scale decreases as the coupling is increased.

\section{B. Crossover scales: DMFT viewpoint}

When used as an approximation for the two-dimensional half-filled Hubbard model of interest here, DMFT yields a symmetry-breaking phase transition into an insulating phase with antiferromagnetic (AF) long-range order at finite temperatures for any value of $U$. This result is expected because DMFT does not take into account spatial fluctuations that destroy finite- $T$ AF ordering in two dimensions (and, hence, does not satisfy the MerminWagner theorem [21,22]). The Néel temperature $T_{\text {Néel }}^{\mathrm{DMFT}}$ obtained within DMFT is displayed in Fig. 6 as a function of $U$ (blue curve in upper panel), as well as the corresponding staggered magnetization $m$ as a function of temperature (lower panel). As expected, the Néel temperature displays a maximum, which signals the crossover between the weak-coupling regime, in which the Néel temperature and magnetization are exponentially small, and the strong-coupling regime. For very large $U$, the charge gap is of order $U$, and the magnetization saturates. In that regime, for $T \ll U$, spin degrees of freedom are described by an effective Heisenberg model with superexchange $J=4 t^{2} / U$, and the DMFT Néel temperature is
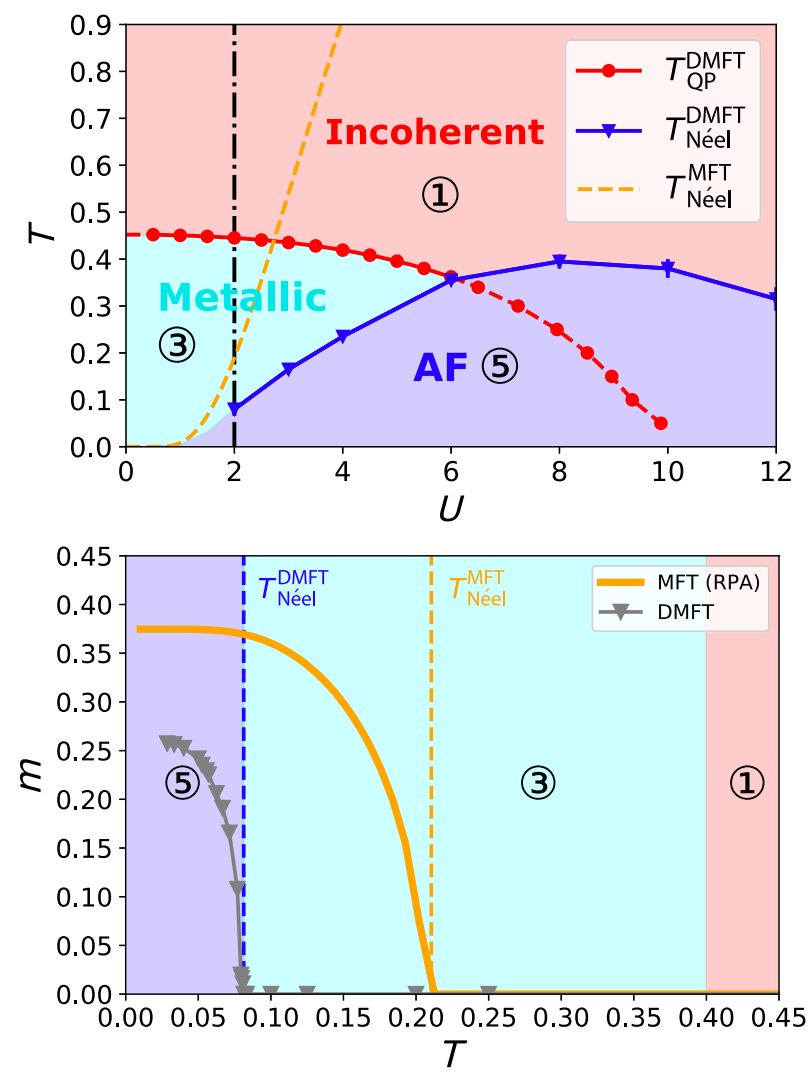

FIG. 6. Upper panel: phase diagram of the half-filled Hubbard model on a square lattice as a function of $U$ within DMFT. The dotted dashed line marks $U=2$. Lower panel: magnetization at $U=2$ as a function of $T$ calculated in (symmetry-broken) DMFT (dark-gray curve) and MFT (orange curve).

proportional to $J$. For reference, in Fig. 6, we also display the result of the standard static mean-field theory (i.e., the Hartree mean field for the transition into the spin-density wave phase, denoted by MFT). MFT considerably overestimates the Néel temperature as well as $m$ for most values of $U$. This overestimation is particularly pronounced at large $U$ where static MFT does not correctly separate spin and charge degrees of freedom, so the ordering temperature incorrectly coincides with the charge gap $U m(\sim U$ at large $U$ ) (for a comparison of MFT and DMFT, see Ref. [65]).

The key observation is that, taken together, the DMFT quasiparticle coherence scale (red line in Fig. 6) and the DMFT Néel temperature (blue line) can be taken as semiquantitative mean-field estimates for the crossover lines separating the different regimes discussed in Fig. 2 above. The DMFT quasiparticle coherence scale is an estimate for the crossover into the metallic regime (3) in Fig. 2 (with $T_{\mathrm{QP}}^{\mathrm{DMFT}} \simeq 0.45$ comparable within error bars to the nodal value from our benchmark, while the antinodal one is about $12 \%$ smaller). In turn, since in this weak-coupling regime the insulating gap is associated with magnetic quasi-long-range order, the DMFT Néel temperature is an estimate for the onset of the insulating behavior-regime (5) in Fig. 2. 


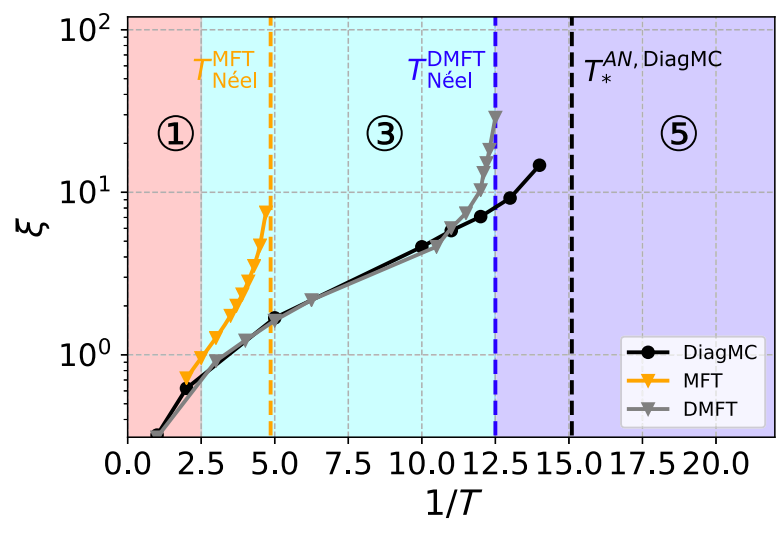

FIG. 7. Magnetic correlation length as a function of (inverse) temperature for DiagMC, MFT, and DMFT on a logarithmic scale.

Note that $T_{\mathrm{Néel}}^{\mathrm{DMFT}} \simeq 0.08$, compared to $T_{*}^{\mathrm{AN}} \simeq 0.065$, while $T_{*}^{N} \simeq 0.0625$ is about $4 \%$ smaller.

The magnetic correlation length can be calculated within both MFT and DMFT. In both cases, this calculation is achieved by using the appropriate Bethe-Salpeter equations for the correlation function: with a static vertex equal to the bare $U$ within MFT (the calculation then reduces to the random phase approximation, RPA) and with a fully frequency-dependent but spatially local vertex within DMFT (see Ref. [28]). It is often overlooked that meanfield methods (MFT or DMFT) provide us with a determination of correlation functions, and indeed, it naively appears as somewhat paradoxical that spatially dependent correlation functions can be obtained from a mean-field ansatz that is local at the one-particle level. This paradox is actually a classic question in statistical mechanics, whose resolution lies in a careful interpretation of linear-response theory [66]. The mean-field correlation lengths are displayed in Fig. 7 in comparison to the DiagMC benchmark. It is apparent that the DMFT correlation length is in excellent agreement with the benchmark down to $T \simeq 0.1$. The figure also clearly illustrates the connection between long-range magnetic correlations and insulating behavior.

\section{Insulating regime}

We finally comment on the comparison between the DMFT self-energies and the true solution in the low- $T$ insulating regime. Within DMFT, the insulating gap in this weak-coupling regime corresponds to a Slater mechanism associated with AF long-range order [20]. It is associated with spin polarization and a nonzero value of the real part of the self-energy (omitting the Hartree term) $\operatorname{Re} \Sigma_{\uparrow}(0)=$ $-\operatorname{Re} \Sigma_{\downarrow}(0) \neq 0$ such that the quasiparticle equation $\omega+$ $\mu-\operatorname{Re} \Sigma(\omega)=0$ has solutions only for $\omega$ outside the gap. The low-frequency behavior of the DMFT self-energy is nonsingular, and in particular, it has (also using particle-

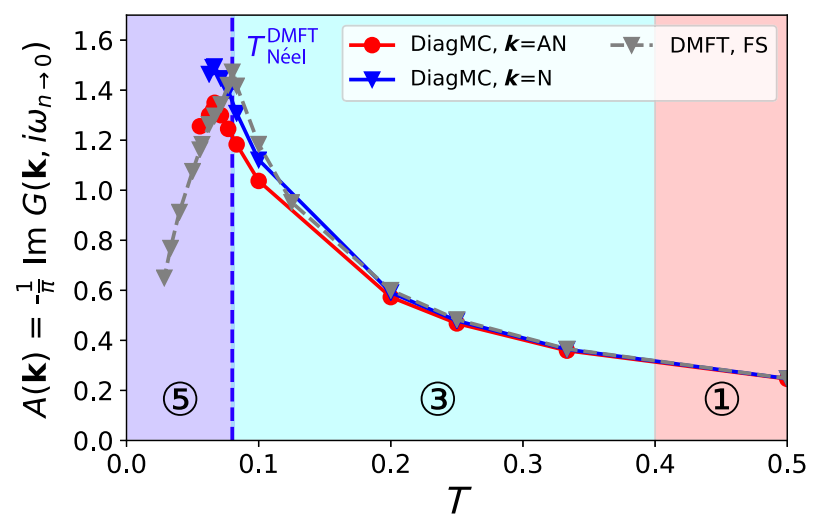

FIG. 8. Spectral function extrapolated to zero frequency at the antinode and node, obtained from the imaginary part of the Green function in DiagMC (solid lines; red circles are for the antinode, and blue triangles are for the node) and DMFT (on the Fermi surface; dashed line with gray triangles).

hole symmetry) a spin-independent linear term $\operatorname{Im} \Sigma\left(i \omega_{n}\right) \propto$ $(1-1 / Z) \omega_{n}+\cdots, \quad$ corresponding to $\operatorname{Re} \Sigma_{\sigma}(\omega)=$ $\operatorname{Re} \Sigma_{\sigma}(0)+(1-1 / Z) \omega+\cdots$ on the real-frequency axis, which is similar in structure in the metallic phase and in the AF-ordered insulating phase. This behavior is clearly apparent in Fig. 4. In contrast, because a spin polarization is absent at any nonzero $T$ in the true solution, the selfenergy must be much more singular at low frequencies to open the insulating gap, as becomes clear from Figs. 3 and 4 . The precise nature of this singularity is discussed in Sec. VII. At $T=0$, however, the system does order, and the general structure of the self-energy is expected to be similar to the Slater (and DMFT) one discussed above.

Finally, in Fig. 8, we display the value of the spectral function extrapolated to zero frequency, as obtained from DMFT as well as from the DiagMC benchmark at the node and antinode. Interestingly, there is rather good agreement between these different methods for this quantity, despite the different low-frequency behavior of the self-energy. In particular, we note that the crossover into the insulating regime is very sharp when seen from this physical observable, which is rather well approximated by the DMFT solution that has long-range order in the insulating regime and hence displays a singularity at the Néel temperature. Detailed analysis close to the crossover temperature would, of course, reveal differences.

Summarizing, we see that DMFT provides a reasonable approximate description of the key crossovers encountered as a function of temperature. As a mean-field theory, it of course mimics the crossover into an insulating phase with a large $\mathrm{AF}$ correlation length as a phase transition into a phase with long-range AF order. Including fluctuations beyond DMFT is therefore especially crucial in two dimensions, in which long-range order exists only at $T=0$. This is the purpose of the cluster and diagrammatic (vertex-based) extensions of DMFT discussed in the following sections. 


\section{INCLUDING FLUCTUATIONS BEYOND MEAN FIELD: SINGLE-PARTICLE PROPERTIES}

In this section, we show how the inclusion of (nonGaussian bosonic) spatial fluctuations influences the oneparticle properties (self-energies) beyond the mean-field picture. This goal can be achieved by several methods: cluster extensions (dynamical cluster approximation DCA, cellular DMFT CDMFT; Sec. IVA) and diagrammatic extensions of DMFT (Sec. IV B), as well as with other approaches (Sec. IV C). In this section, the results for the self-energy are presented and compared to the exact (benchmark) methods.

\section{A. Cluster extensions of DMFT: DCA and CDMFT}

Quantum cluster methods [29] are quite natural extensions of DMFT in which the nonlocal components of the self-energy are computed by considering a cluster of $N_{c}$ sites self-consistently embedded in the lattice. These methods provide a controlled sequence of approximations that converge to the exact result when $N_{c} \rightarrow \infty$. Depending on the regime of parameters, convergence in this asymptotic limit may or may not be attained in practice, however.

There are several flavors available within the broad family of quantum cluster theories, depending on how, exactly, the interacting cluster is described and how the embedding is performed. First, we consider the dynamical cluster approximation (DCA, Refs. [67-69]), where the Brillouin zone is paved with patches, and the self-energy is approximated as a piecewise constant function over these patches whose components are calculated from a cluster of $N_{c}$ sites with periodic boundary conditions, hence defining the discrete momenta associated with each patch. For methodological details, see Ref. [29] and Appendix D 5.

Figure 9 (left) shows the self-energy on the Matsubara axis of a DCA calculation with $N_{c}=128$ momentum patches for several temperatures and the benchmark DiagMC data as reference. For these calculations, symmetry breaking to an AF long-range-ordered phase was not allowed. We see that the improvement over single-site DMFT by these calculations is that the momentum dependence of the quasiparticle coherence scale along the Fermi surface and nodal-antinodal differentiation is quantitatively reproduced (regimes (1) (3)).

However, the (nonmagnetic) DCA with $N_{c}=128$ is not able to open the pseudogap at low temperatures (shown in Fig. 9 until $T=0.067$, i.e., $\beta=1 / T=16$ ). The reason for this failure is easily understood by noting that the correlation length, previously displayed in Fig. 7 , is $\xi \approx 15$ lattice sites at the lowest $T$ available $(\beta=14)$ and even larger at lower $T$. A periodic cluster of finite-size $N_{c}$ cannot resolve a correlation length larger than $\xi_{\mathrm{DCA}} \sim \sqrt{N_{c}} / 2$ which is $\simeq 5.7$ lattice spacings for $N_{c}=128$. From this argument, one would expect that a cluster of order 1000 sites would be required to capture the opening of the insulating pseudogap with DCA in the case at hand. As discussed in Appendix D 5, this computation is beyond practical reach of the algorithm that we use here in the context of DCA.

A different strategy in the family of cluster extensions of DMFT is to consider a real-space embedding and hence a cluster with open boundary conditions, as in the CDMFT approach $[32,70]$. Figure 9 also displays data from CDMFT with $N_{c}=64=8 \times 8$. As apparent from the data, the real-space embedding method again captures the momentum differentiation of the coherence scale but fails in opening the pseudogap for the same reasons as DCA $\left(\xi_{\mathrm{CDMFT}} \approx 8 / 2=4\right)$. From a quantitative perspective, a recently introduced extrapolation scheme (center-focused extrapolation, CDMFT + CFE [71]; Appendix D 6) improves the comparison with the benchmark; however, for the $U=2$ case, it also fails to open a gap. Hence, the size of the clusters has to be increased much beyond the
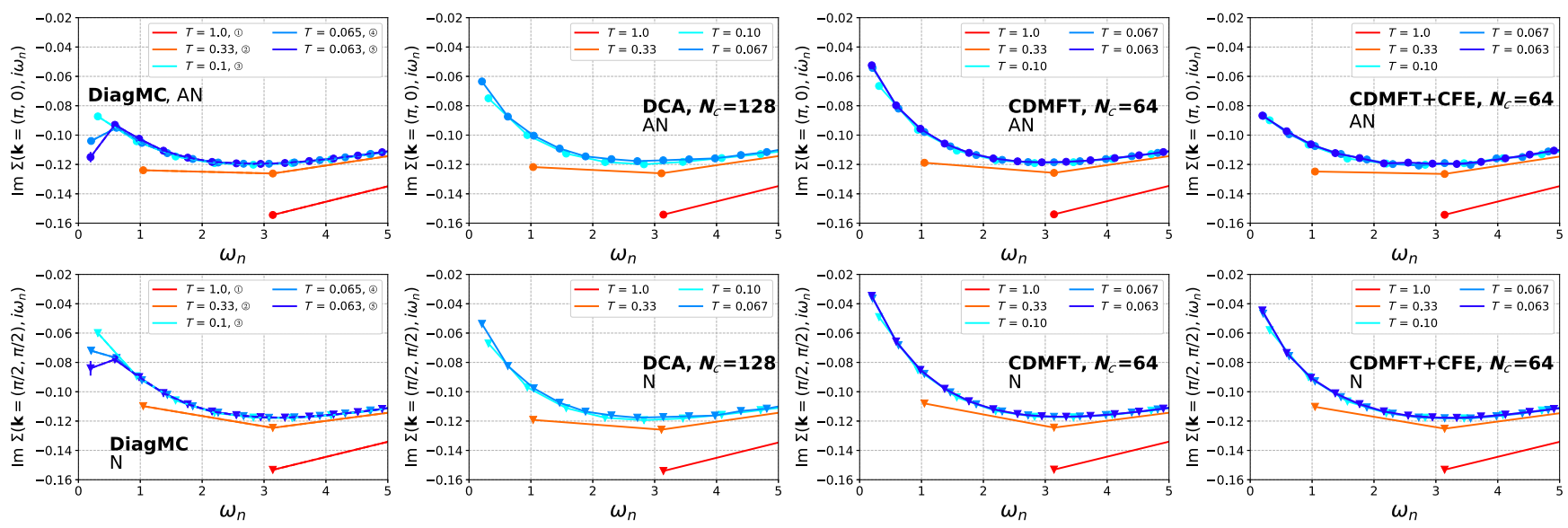

FIG. 9. Imaginary part of the self-energy for the antinode (upper row) and node (lower row) as a function of Matsubara frequencies for DiagMC (first panel on the left-hand side), DCA $\left(N_{c}=128\right.$, second panel), CDMFT $\left(N_{c}=64\right.$, third panel), and center-focused extrapolated CDMFT (CDMFT $+\mathrm{CFE}, N_{c}=64$, fourth panel). 
values of $N_{c}$ considered here in order to reproduce the pseudogap at this small interaction strength (see also Ref. [48]).

Summarizing, we conclude that extensions of DMFT based on cluster embedding methods succeed in reproducing the momentum dependence of the quasiparticle coherence scale (nodal-antinodal differentiation) in the metallic regime but that much larger cluster sizes would be necessary in order to capture the opening of the insulating pseudogap due to the very large correlation length in this weak-coupling regime. Cluster embedding approaches perform much better in regimes with a smaller correlation length, such as larger values of $U$ (i.e., in the strongcoupling regime) [72] or (disregarding the sign problem) when doping away from half-filling, as documented by the success of these methods in capturing the strong-coupling pseudogap of the doped Hubbard model [31,73-91]. Let us also note here that, analogously to what was done with DMFT in Fig. 8, allowing for AF order within these cluster methods-and hence mimicking the large correlation length regime as an ordered state-would most likely improve the agreement; see also Ref. [92]. We do not attempt this here-see, however, Sec. VI for additional information about ordering within CDMFT.

\section{B. DTA, TRILEX, DF and DB}

In view of the limitations of cluster embedding theories for this $U=2$ weak-coupling regime, we now turn to an alternative way of treating long-ranged correlations more efficiently here, namely, diagrammatic extensions of DMFT.

In Fig. 10, we display the results of several such diagrammatic extensions for the frequency dependence of the self-energy at the antinode at different temperatures (apart from the diagrammatic extensions of DMFT, other approximation methods are also considered; see next subsection). Figure 11 displays the results for the nodal point. Note that the lowest temperature displayed is not always the same for the different methods, and it is useful to refer to Table I as a reminder of the important crossover scales. For the sake of comparison, the first panel shows, again, the data from the DiagMC benchmark.

We observe that all of the diagrammatic extensions of DMFT presented here [ladder DГA with a Moriya $\lambda$ correction in the spin channel (Appendix D6a), TRILEX $\Lambda^{2}$ (Appendix D 8), ladder DF (Appendix D 9), and single-shot DB (note that, in the absence of the nonlocal interaction, the fully self-consistent DB theory would coincide with the DF approach when the bosonic hybridization function is discarded; see Appendix D 9)] are
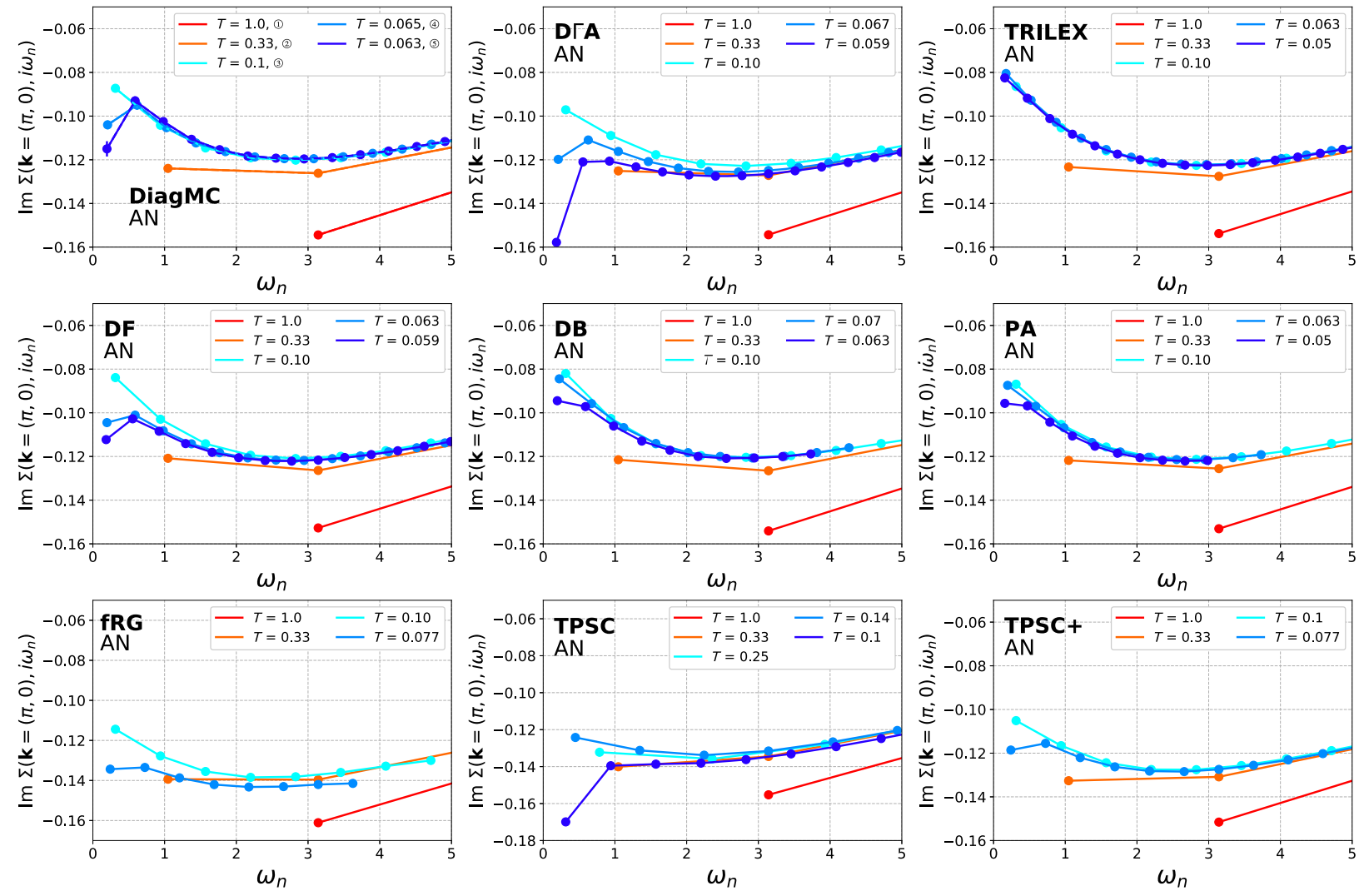

FIG. 10. Imaginary parts of the self-energies at the antinode as a function of Matsubara frequencies calculated by various many-body methods. Note that the lowest temperatures shown sometimes differ for the respective methods in order to show as many of the respective temperature regimes as possible. Also note that the vertical axis is different for the last row of three figures. 

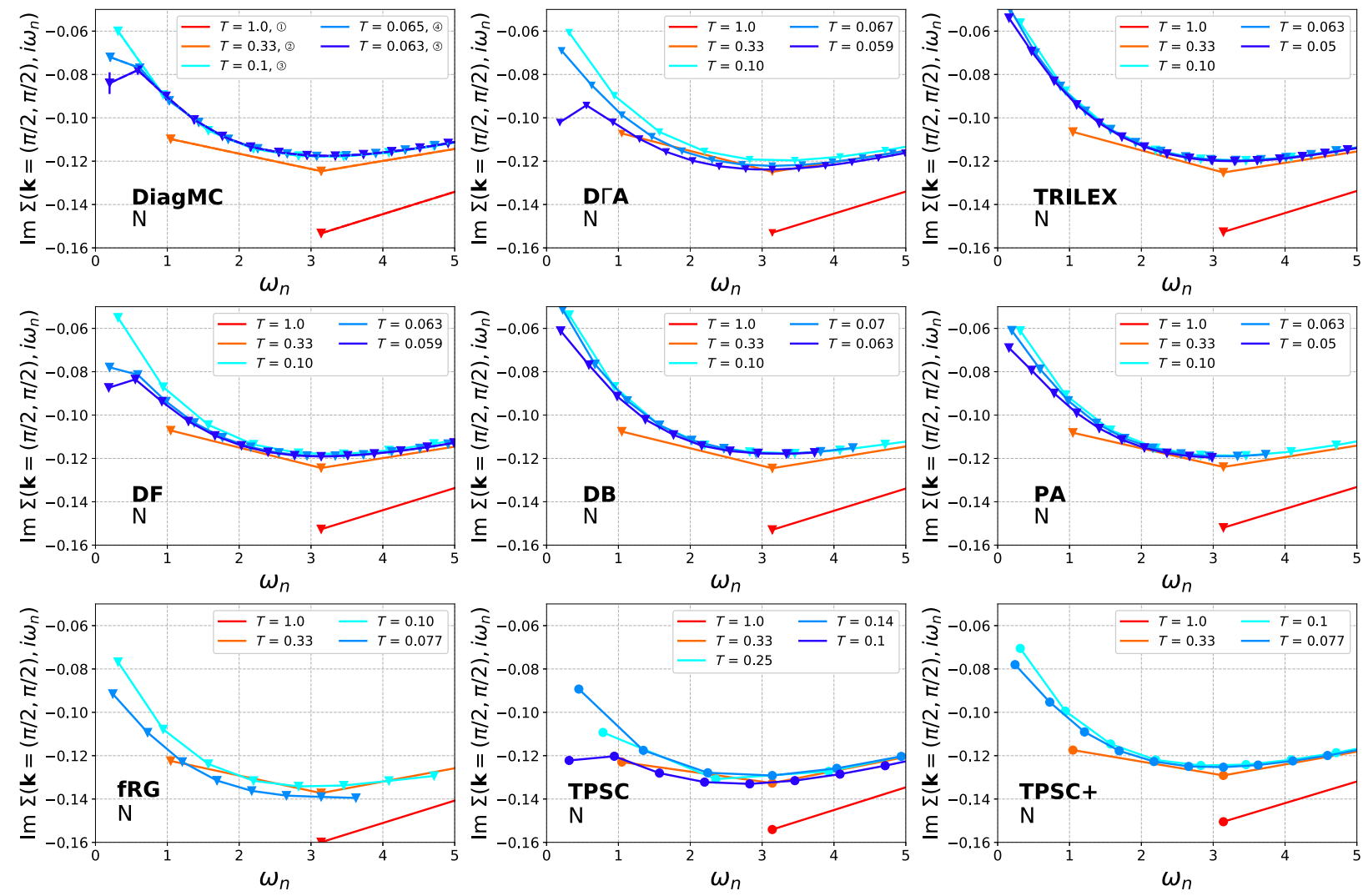

FIG. 11. Imaginary parts of the self-energies at the node as a function of Matsubara frequencies calculated by various many-body methods. Note that the lowest temperatures shown sometimes differ for the respective methods in order to show as many of the respective temperature regimes as possible.

able to correctly reproduce the crossover from the incoherent to the metallic regime. Indeed, all methods display incoherent behavior (region (1) at high temperatures before the onset of quasiparticles becomes visible-first for the nodal point (region (2) and then, at lower temperatures, for the antinode (region (3). The temperatures of this onset $T_{\mathrm{QP}}$, only slightly, if at all, deviate from each other and the benchmarks within the numerical accuracy.

Larger deviations, both on a qualitative and quantitative level, become visible, however, when lowering the temperature into the insulating pseudogap regime associated with growing magnetic correlations. Let us remind the reader that this crossover is signaled by a second change of slope in the self-energies-first, at the antinode (region (4) and, then, at the node (region (5) — corresponding to a scattering rate that grows upon cooling.

Whereas DГA and DF correctly reproduce these crossovers into the pseudogap regime, TRILEX does not exhibit these changes of slope, down to the lowest temperatures where the method could be converged. The DF method also succeeds quantitatively, both at the node and at the antinode, while the DB method appears to perform better at the antinode than at the node (but does not open the gap at the accessible temperatures). From a more quantitative point of view, DF and DB slightly underestimate the scattering rate at the node with respect to DiagMC, whereas DГA seems to slightly overestimate the scattering rate at the antinode and simultaneously exhibits a slightly lower $T_{*}^{N}$ than the benchmark.

Summarizing, we conclude that among the diagrammatic extensions of DMFT presented here, the DГA and the DF method appear to be best at capturing the different crossover regimes for the self-energy. In terms of the practical ability of performing calculations in this parameter regime, we must point out that all methods suffer from convergence problems when going down to lower and lower temperatures. The reason for these difficulties varies from method to method. For the benchmark method DiagMC, the series cannot be summed at low $T$, and the DQMC suffers from the exponentially growing correlation length for $T<T_{\min } \approx 0.063$. In the case of the $\mathrm{D \Gamma A}\left(T_{\min } \approx 0.05\right)$, lower temperatures can be reached if one is able to converge in the internal momentum grids. The same is true for TRILEX $\left(T_{\min } \approx 0.05\right)$, DF $\left(T_{\min } \approx 0.05\right)$, and $\mathrm{DB}\left(T_{\min } \approx 0.063\right)$. Also note that within DiagMC, the lowest reachable temperature is different for the node and antinode $\left(1 / T_{\min }^{\mathrm{AN}}=18 \mathrm{vs} 1 / T_{\min }^{N}=16\right)$.

\section{Other approaches: TPSC, TPSC+, fRG, and PA}

Figures 10 and 11 also show results for three other methods: TPSC/TPSC+, fRG, and PA. Similarly to 
diagrammatic extensions of DMFT, all of these approaches are able to reproduce the two distinct quasiparticle coherence scales $T_{\mathrm{QP}}^{N, \mathrm{AN}}$ at the node and antinode. However, there are significant deviations from the benchmark regarding the onset of the insulating pseudogap behavior.

TPSC is one of the first methods in which a detailed understanding of the mechanism responsible for the weakcoupling pseudogap was achieved early on (see Refs. [34,93,94] and Sec. VII). As seen from Figs. 10 and 11, the change of slope in the self-energies associated with the pseudogap opening is indeed qualitatively captured by TPSC, but the onset temperatures $T_{*}^{\mathrm{AN}, N}$ are severely overestimated. As discussed in Sec. VI, this is due to an overestimation of spin fluctuations in this method. A recent variant of the method, TPSC+ [36], leads to a definite improvement in this respect, as shown in the figures. TPSC + partially feeds the self-energy back into the fluctuation propagators, mimicking frequency-dependent vertex corrections. The PA appears to eventually capture the insulating behavior at the antinode, although at lower temperatures, $T<0.05$, in comparison to DiagMC, but does not open a pseudogap at the node at this temperature.

The fRG calculations are possible only down to a "pseudocritical" temperature scale $T \simeq 0.07$ at which the running coupling constants diverge (see, also, the discussion in Ref. [59]). Down to this temperature, however, fRG is in qualitative agreement with the benchmark and shows a nonmetallic behavior at the antinode (regime (4)).

\section{DOUBLE OCCUPANCY AND POMERANCHUK EFFECT}

In view of its physical significance discussed below, in this section, we present the temperature dependence of the double occupancy:

$$
D=\left\langle n_{\uparrow} n_{\downarrow}\right\rangle .
$$

It is displayed as a function of temperature in Fig. 12, as obtained from different methods. We see (left panel, DQMC and DiagMC benchmarks) that there are three regimes: At high $T$ (down to about $T \simeq 1$ ), $D(T)$ decreases upon cooling and then reaches a minimum; at intermediate temperatures, $D(T)$ actually increases upon cooling; and, finally, $D(T)$ sharply drops when entering the gapped regime. The high- $T$ regime is expected and easy to understand: As $T$ is raised, an increasing number of high-energy doubly occupied configurations are thermally populated, which is apparent from the simple expression of $D(T)$ in the atomic (zero hopping) limit:

$$
D_{\text {at }}=\frac{1}{2+2 \exp (U /(2 T))},
$$

which approaches $D_{0}=0.25$ for $U / T \rightarrow 0$, i.e., at very high temperatures or, alternatively, in the noninteracting limit. The intermediate regime in which $D(T)$ increases upon cooling is more interesting - note that this regime includes, in particular, large parts of the metallic region (3). As observed early on in Ref. [95], this apparently counterintuitive nonmonotonic behavior of $D(T)$ can be understood qualitatively from entropy considerations. Observing that the entropy $S$ is obtained from the free energy $F$ as $S=-(\partial F / \partial T)$ and that $D=(\partial F / \partial U)$, one obtains the thermodynamic Maxwell relation:

$$
\left.\frac{\partial D}{\partial T}\right|_{U}=-\left.\frac{\partial S}{\partial U}\right|_{T} .
$$

Increasing $U$ at fixed temperature in the metallic regime leads to an increase in entropy. Indeed, in this regime, the entropy is linear in $T$, with a slope related to the effective mass, which grows as $U$ is increased. Hence, the right-hand side of Eq. (5) is negative, and thus $D(T)$ must increase upon cooling or decrease upon heating in this regime. This phenomenon is the same as the famous "Pomeranchuk effect" in liquid ${ }^{3} \mathrm{He}$ (Clausius-Clapeyron equation): Upon heating the system, a tendency to increased localization (smaller $D$ ) is found because localization leads to a higher (spin) entropy and is thus thermodynamically favorable (until thermal population of doubly occupied sites kicks in at higher $T$ ). Note that the sign of $\partial D / \partial T$ also directly determines the shape of the isentropic lines in the $(U, T)$ plane, which are defined by $S\left(T_{i}(U), U\right)=$ const and hence obey $[96,97]$
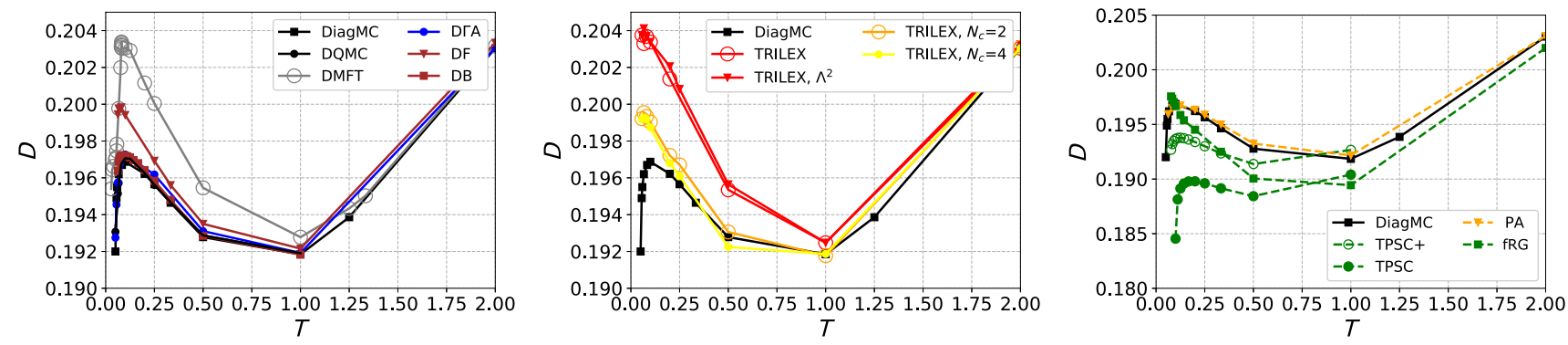

FIG. 12. Double occupancy $D$ as a function of temperature from various methods. 


$$
c\left(T_{i}\right) \frac{\partial T_{i}}{\partial U}=\left.T_{i} \frac{\partial D}{\partial T}\right|_{T=T_{i}},
$$

with $c(T)=T(\partial S / \partial T)$ representing the specific heat per lattice site. Hence, cooling can, in principle, be achieved by increasing the interaction strength adiabatically in the regime where $\partial D / \partial T<0$, as initially suggested in Ref. [96], further discussed in Ref. [97], and experimentally realized in cold atomic systems with extended $S U(6)$ symmetry [98].

Finally, the low- $T$ behavior in which $D$ sharply decreases again upon cooling corresponds to temperatures around regime (4) in which the system behaves as an antiferromagnetc insulator. We note that the total energy of the Hubbard model is given by

$$
\begin{aligned}
\langle H\rangle & =-t \sum_{\langle i j\rangle, \sigma}\left\langle c_{i \sigma}^{\dagger} c_{j \sigma}\right\rangle+U \sum_{i}\left\langle n_{i \uparrow} n_{i \downarrow}\right\rangle \\
& =E_{\mathrm{kin}}+E_{\mathrm{pot}},
\end{aligned}
$$

so that $D=E_{\mathrm{pot}} / U$. The drop in $D$ can be understood from the fact that at small $U$, the crossover into the (Slater) antiferromagnetic correlation regime corresponds to a gain in potential energy [47,51,92]. It was shown in Ref. [92] that at strong coupling, in contrast, $D$ increases when entering the (Heisenberg) antiferromagnetic correlations regime, corresponding to a gain in kinetic energy.

We see (left panel of Fig. 12) that DMFT reproduces all three regimes qualitatively but overestimates the amplitude of the Pomeranchuk effect by approximately a factor of 2 , as discussed in Ref. [97]. The reason is that the spin entropy in the localized state at high $T$ is overestimated in DMFT due to its neglect of spatial correlations. Obviously, in DMFT, the sharp drop at low $T$ corresponds to the phase transition into an ordered phase-a mean-field description of the actual crossover (see above). This drop is also directly related to an increase of the local moment since via $\left\langle S_{z}^{2}\right\rangle=1-2 D$ the double occupancy is related to the increase of AF correlations.

All the methods displayed in Fig. 12 qualitatively reproduce the nonmonotonous behavior of $D(T)$, but the sharp drop at low $T$ is, as expected, only present in the methods that can describe the low- $T$ antiferromagnetic insulator regime. For this reason, it is absent in the TRILEX-based methods for the range of temperatures studied (middle panel). Single-site TRILEX also overestimates $D$ significantly, but cluster extensions of TRILEX are closer to the benchmark at intermediate $T$. DF similarly overestimates the double occupancy. For details, including differences between different variants of these "dual" methods, see the discussion about selfconsistency in Appendix D 9. Note that fRG qualitatively captures the Pomeranchuk effect. In the present implementation, however, it does not display a drop at low temperatures.

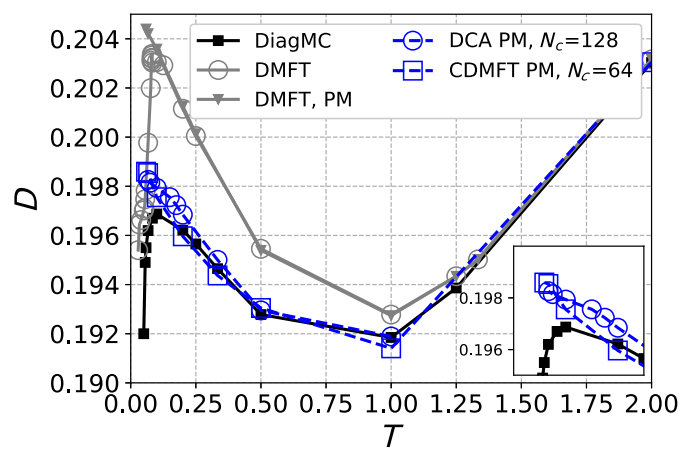

FIG. 13. Results for the double occupancy from DMFT, DCA, and CDMFT compared to DiagMC. For DMFT, we display both the (metastable) solution obtained by constraining the system not to order (PM) and the lower free-energy solution with AF magnetic ordering. The zoom in the inset confirms the absence of a downturn in the cluster techniques when constrained to the PM solution due to the insufficient cluster size.

In contrast, $\mathrm{DГA} \mathrm{and} \mathrm{DB}$ (single shot) follow the benchmark quite accurately. Also, the PA appears to capture all three described regimes, while TPSC and TPSC + qualitatively predict the correct physical picture, albeit it is less accurate quantitatively than PA in comparison to the benchmarks.

The above results suggest that long-range magnetic correlations are the cause of the downturn of the double occupancy at low temperatures. To further establish this point, in Fig. 13, we display the double occupancy vs temperature calculated with DMFT by either constraining the solution to remain paramagnetic or allowing for the solution with long-range AF order (which is the lowerenergy stable solution at low $T$ within DMFT). One sees that only the latter displays the downturn, emphasizing that the AF-ordered solution, despite violating the MerminWagner theorem, is a better approximation to thermodynamic quantities in a regime where the correlation length is large. We also display in Fig. 13 the results obtained with DCA and CDMFT, restricted to the PM solution. In that case, as expected, the downturn is not reproduced since the cluster sizes investigated here are too small compared to the correlation length (see also the discussion in Sec. IVA).

We end this section with a technical remark on the actual calculation of $D$ in the investigated methods. In DQMC and DiagMC, $D$ can be directly calculated as an equal-time correlation function. For the other methods, the calculation of the double occupancy is possible either via the (lattice) Green function and self-energy, utilizing the equation-of-motion technique based on the Galitskii-Migdal formula [99],

$$
D=\frac{T}{U} \sum_{\mathbf{k}, \omega_{n}} \Sigma\left(\mathbf{k}, i \omega_{n}\right) G\left(\mathbf{k}, i \omega_{n}\right)
$$

(used in the DГA, DF, PA, and TPSC/TPSC+; for details on the latter; see Appendix D 10), or, when allowed by the 
algorithm used for the impurity solver, from a direct computation on the impurity (for DMFT, its cluster extensions and TRILEX; see also Ref. [100]). The DB result was obtained via the local part of the lattice susceptibility as discussed in Ref. [100]. For a comparison to self-consistent $\mathrm{DB}$, we refer to Appendix D 9. For details on the calculation of $D$ in fRG, we refer to Appendix D 11. We note in passing that, when applying Eq. (8), treating the high-frequency tails is very important to obtain accurate results.

\section{INCLUDING FLUCTUATIONS BEYOND MEAN FIELD: MAGNETIC CORRELATIONS}

In this section, we turn to two-particle correlation functions and compare the different methods for two important observables probing the magnetic correlations: the static antiferromagnetic spin susceptibility $\chi_{\mathrm{sp}}\left(\mathbf{q}=(\pi, \pi), i \Omega_{n}=0\right)$ and the magnetic correlation length $\xi$.

\section{A. Antiferromagnetic static spin susceptibility}

The spin susceptibility (or spin correlation function) is given by

$\chi_{\mathrm{sp}}\left(\mathbf{q}, i \Omega_{n}\right)=\int_{0}^{\beta} d \tau \sum_{\mathbf{r}} e^{i \tau \Omega_{n}} e^{-i \mathbf{q r}}\left\langle S_{z}(\mathbf{r}, \tau) S_{z}(0,0)\right\rangle$,

where we define

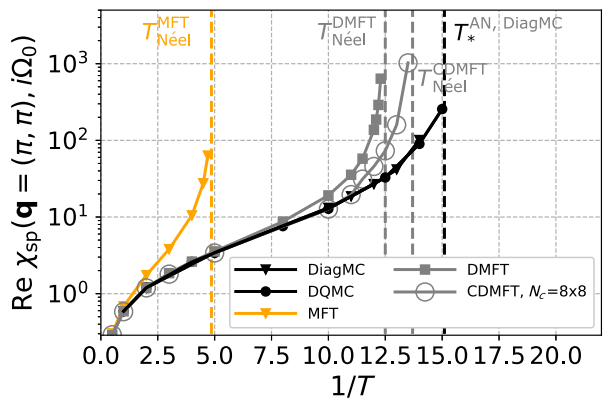

FIG. 14. Antiferromagnetic static susceptibility $\chi_{\mathrm{sp}}(\mathbf{q}=$ $\left.(\pi, \pi), i \Omega_{n}=0\right)$ as a function of (inverse) temperature for various methods on a logarithmic scale.

$$
S_{z}(\mathbf{r}, \tau)=n_{\uparrow}(\mathbf{r}, \tau)-n_{\downarrow}(\mathbf{r}, \tau),
$$

and $\beta=1 / T$. Note that we omit, for simplicity, an additional prefactor of $1 / 2$ for the spin operator and that $\left\langle n_{\uparrow}\right\rangle=\left\langle n_{\downarrow}\right\rangle=$ 0.5 at half-filling in the paramagnetic phase $(T \neq 0)$.

The temperature dependence of the static spin susceptibility $\chi_{\mathrm{sp}}\left(\mathbf{q}, i \Omega_{n}=0\right)$ is of particular interest for the case of the half-filled Hubbard model on the square lattice, as the perfect nesting (see Fig. 1) leads to a strong enhancement at $\mathbf{Q}=(\pi, \pi)$. The temperature dependence of $\chi_{\mathrm{sp}}(\mathbf{q}=\mathbf{Q}$, $\left.i \Omega_{n}=0\right)$ reflects the increasing dominance of antiferromagnetic spin fluctuations upon cooling. One starts at high $T$ with almost independent fluctuating moments and a Curie law $\chi_{\text {sp }}\left(\mathbf{q}=\mathbf{Q}, i \Omega_{n}=0\right) \propto T^{-1}$ (bosonic mean-field behavior). Approaching the $T=0$ ground state with antiferromagnetic long-range order, the range of spin correlations grows, and nonlocal spin fluctuations in the paramagnetic phase (antiferromagnetic paramagnons) develop. At low $T$, a regime with an exponentially growing correlation length is found (see below).

Figures 14 and 15 display $\chi_{\mathrm{sp}}\left(\mathbf{q}=\mathbf{Q}, i \Omega_{n}=0\right)$ for various methods as a function of (inverse) temperatures on a logarithmic scale. We start our analysis with Fig. 14. As already described in the mean-field picture in Sec. III, both $\mathrm{MFT}\left(T_{\mathrm{N} \text { éel }}^{\mathrm{MFT}} \approx 0.21\right.$, orange triangles) and DMFT $\left(T_{\text {Néel }}^{\text {DMFT }} \approx 0.08\right.$, gray squares), due to their mean-field nature, incorrectly predict finite Néel temperatures: The crossover is mimicked as a true (continuous) phase transition (left panel). The thermodynamic transition manifests itself as a divergence of the susceptibility at the corresponding wave vector.

Of course, the Mermin-Wagner theorem prohibits finitetemperature ordering in $2 \mathrm{D}$, which is reflected in the data of both benchmark methods DiagMC (black triangles) and DQMC (black circles), whose susceptibilities do not diverge at finite $T$. Rather, after following the mean-field curves at high temperatures, the benchmark data enter an intermediate regime in which $\chi(\mathbf{Q})$ appears to increase approximately exponentially. This regime largely coincides with the metallic regime (3), eventually crossing over into a second exponential regime at low $T$ (see also Sec. VIC), which sets in at a temperature close to the DMFT Néel
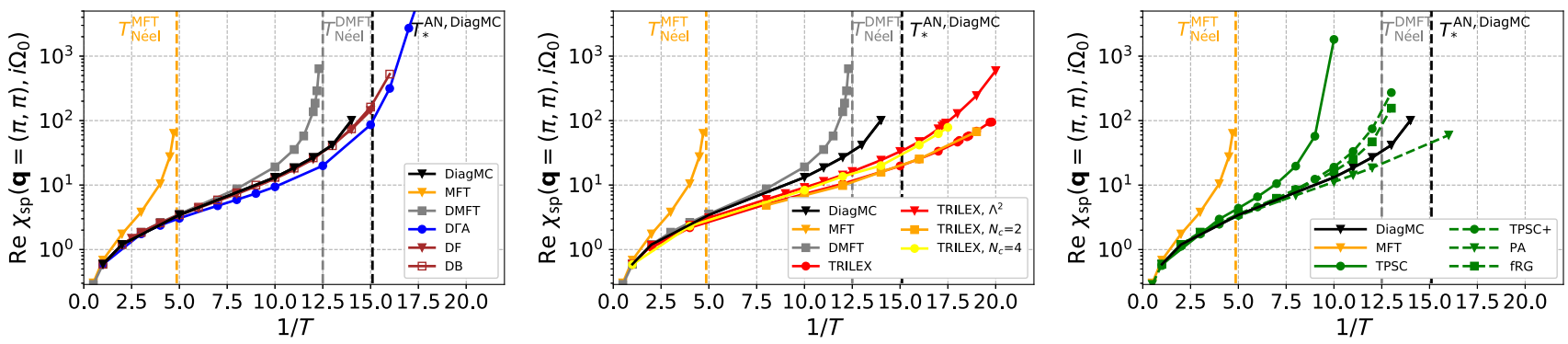

FIG. 15. Antiferromagnetic static susceptibility $\chi_{\mathrm{sp}}\left(\mathbf{q}=(\pi, \pi), i \Omega_{n}=0\right)$ as a function of (inverse) temperature for various methods on a logarithmic scale (continued). 
ordering. This low- $T$ exponential regime is to be expected since, there, the charge degrees of freedom are frozen out by the gap and the system enters the insulating regime (5, as indicated by the black dashed line, $T_{*}^{\mathrm{AN} \text {,DiagMC }}$. In this regime, the effective spin dynamics is expected to be described by a nonlinear sigma model, and this exponential growth is typical of the lower critical dimension $d=2$ [34,101-104]. The first exponential observed in the metallic regime is more surprising and will be discussed in more detail below. Let us stress that, because of the reasons already mentioned for single-particle quantities, both benchmark methods are limited in terms of the temperatures they can reach $\left(T_{\min } \approx 0.07\right)$. Note that the lowest reachable temperature can differ from that for the selfenergy.

The inclusion of short-range correlations with CDMFT leads to (i) a quantitative agreement with the benchmark until $T \approx 0.1$ and (ii) only a slight drop of the Néel temperature in comparison to DMFT, to $T_{\text {Néel }} \approx 0.073$. In principle, as the cluster extensions of DMFT are controlled methods, the Mermin-Wagner theorem is restored in the infinite-cluster-size limit. However, this restoration has been shown to be logarithmic in the strongcoupling regime [105], which is in agreement with this very small change in $T_{\text {Néel }}$ (see also Ref. [71]).

Turning to the diagrammatic extensions of DMFT, in the left panel of Fig. 15, one can see that DГА (left panel), which respects the Mermin-Wagner theorem [51,53,54,106], captures well the different regimes of the benchmark (Curie law at high temperatures and the two exponential regimes). The small quantitative underestimation observed here may potentially be cured by an improved version of the Moriya $\lambda$ correction [51] or a more thorough treatment of the asymptotics of the vertex function as a function of frequency [107-109]. DF and single-shot DB agree well with the benchmark where the algorithm can be reliably converged.

In the case of TRILEX (central panel), we present results for different variants of the method, all of which seem to capture a low-temperature exponential scaling, however, with different degrees of accuracy: Whereas single-site TRILEX (red circles, solid line) and cluster TRILEX with two cluster sites (TRILEX $N_{c}=2$, orange squares) largely underestimate the values of the susceptibility, the result is significantly improved by increasing the cluster size to four (TRILEX $N_{c}=4$, yellow circles). Remarkably, the TRILEX variant with the electron-boson vertex inserted on both sides (TRILEX $\Lambda^{2}$, red triangles; see Appendix D 8) seems to be on top of the cluster $N_{c}=4$ results. Investigating how these values eventually converge to the exact results with $N_{c} \rightarrow \infty$ is left for future studies.

We finally turn to the other methods (right panel): Both PA and fRG quantitatively capture the high- $T$ Curie regime. The PA appears to systematically underestimate $\chi_{\text {sp }}$ from $1 / T=10$ on, whereas fRG overestimates it. Let us comment here that, although the presented fRG scheme does not fulfill the Mermin-Wagner theorem, its recent multiloop extension [56-58] and the PA do [60,62] (see also Ref. [55]). Both TPSC and TPSC+ are in agreement with the benchmarks at high temperature, but, as already mentioned in the previous section about the self-energy, the spin-fluctuation intensity is significantly overestimated in TPSC with respect to the benchmark (right panel). This overestimation is improved again in the TPSC+.

Our results also demonstrate that, remarkably, neither the fact that the Mermin-Wagner theorem is respected by a theory nor that a theory uses self-consistent interacting Green functions guarantees a quantitatively better result, as can be inferred by a comparison of the TPSC or PA result with the benchmark, respectively.

\section{B. Magnetic correlation length}

The spatial range of antiferromagnetic spin correlations can be quantified by the magnetic correlation length $\xi$, which, in practice, can be extracted by a fitting procedure using the Ornstein-Zernike $(\mathrm{OZ})$ form of the bosonic propagator [110]:

$$
\chi_{\mathrm{sp}}\left(\mathbf{q}, i \Omega_{n}=0\right)=\frac{A}{(\mathbf{q}-\mathbf{Q})^{2}+\xi^{-2}} .
$$

The results presented in this section were obtained using a slightly modified form of the OZ expression, appropriate for a model on a lattice, as described in Appendix A. Note that we neglect (small) deviations from the OZ form beyond mean field. Within the OZ form, the AF static susceptibility discussed previously obeys $\chi\left(\mathbf{Q}, i \Omega_{n}=0\right) \sim A \xi^{2}$.

Figure 16 shows the correlation length $\xi$ obtained via such a fitting procedure applied to the susceptibility data from the different methods. One can immediately see that the curves are, to a large extent, qualitatively similar to the susceptibility curves of Fig. 15. Interestingly, the intermediate- $T$ exponential behavior in the metallic regime is clearly visible in the DiagMC benchmark data. The lowest$T$ exponential regime in the insulator is hard to reach with DiagMC, but it is obtained in DГА (and, less clearly, in PA and TRILEX), which can be used down to lower temperature than the benchmark methods. We note, however, that sizable quantitative differences do exist between the different methods at low $T$, and hence we conclude that the precise determination of the correlation length in the low- $T$ regime, where it becomes exponentially large, is a challenge for all state-of-the-art computational methods currently available.

\section{Three regimes of magnetic correlations}

Summarizing, we observe three successive regimes for magnetic correlations as the temperature is lowered. At high $T(\gtrsim 1 / 5)$, a Curie mean-field behavior is found with a small correlation length $(\lesssim 2)$, and all methods (except static MFT) are basically in quantitative agreement in this 

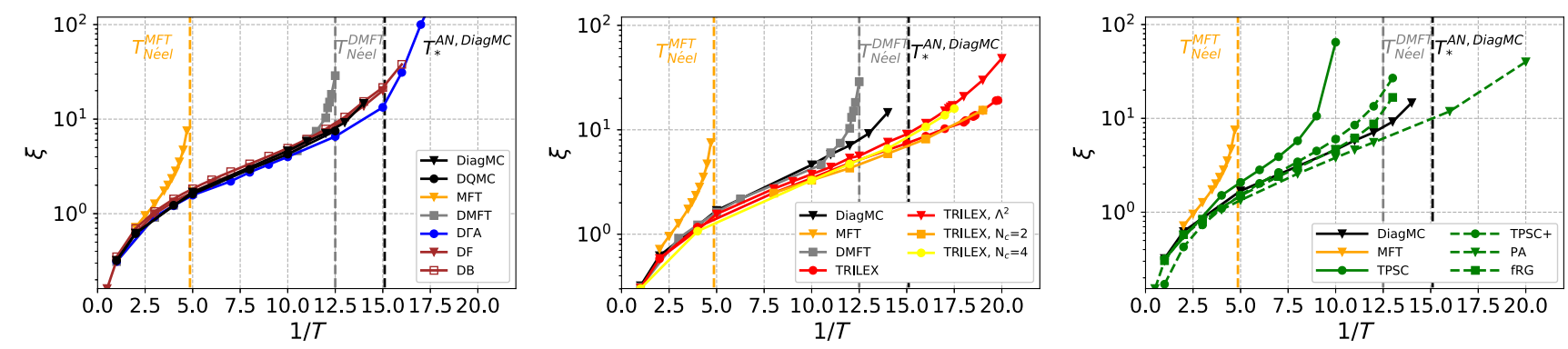

FIG. 16. Magnetic correlation lengths $\xi$ extracted from the magnetic susceptibility as a function of (inverse) temperature for various methods on a logarithmic scale.

regime. At intermediate temperatures $1 / 5 \gtrsim T \gtrsim 1 / 12.5 \simeq$ $T_{\mathrm{Néel}}^{\mathrm{DMFT}}$, we find a correlation length that appears to increase exponentially to a good approximation, with values $\xi(T=$ $1 / 5) \simeq 2$ reaching $\xi(T=1 / 13) \simeq 10$. This result is somewhat surprising since this regime is metallic with quasiparticles that become more coherent as the temperature is lowered, at least in the nodal region. We further discuss the physical significance of this finding in Sec. VII by comparing to spin-fluctuation theories. All methods reproduce this intermediate exponential regime qualitatively. On the quantitative level, excellent agreement is found between the benchmark DiagMC and, especially, DF and DB throughout this regime. We also note very good agreement for the determination of the correlation length with DMFT and TPSC+ down to $T \simeq 1 / 10$. Most other methods (DГA, the various variants of TRILEX, and the PA) also provide a satisfactory determination of the susceptibility and correlation length in this intermediate regime.

The low-temperature insulating regime $T \lesssim 1 / 12.5 \simeq$ $T_{\text {Néel }}^{\mathrm{DMFT}}$ is challenging to probe with current state-of-theart benchmark methods. The DГA results down to $T=$ $1 / 20$ are consistent with the exponential growth of the correlation length [47,51], expected from the low-energy description of the spin degrees of freedom by a nonlinear sigma model once a charge (pseudo)gap opens up. We note significant discrepancies between the different approximate methods available in this regime, however. The growth rate of this exponential regime appears to be different (and faster) than the one in the intermediate- $T$ metallic regime, a finding that will have to be confirmed in future work by exact computational methods when they become capable of reaching lower temperatures.

\section{INSIGHTS INTO THE NATURE AND ROLE OF SPIN FLUCTUATIONS}

In this section, we provide understanding into the physical nature of the different regimes highlighted above, especially the metallic regime and the (pseudo)gapped insulating regime. We focus, in particular, on the nature and role of spin fluctuations. We explore whether spinfluctuation theory of the weak-coupling type is able to qualitatively describe the various regimes: This analysis also provides analytical insights into the physics of these different regimes. In particular, we focus on the following key questions:

(i) What is the physical mechanism for opening the pseudogap at low temperature?

(ii) What are the implications of the growing antiferromagnetic correlation length upon cooling for the coherence of quasiparticles in the metallic regime?

(iii) To what extent can this metallic regime be characterized as a Fermi liquid?

\section{A. Weak-coupling spin-fluctuation theory}

In this subsection, we interrogate weak-coupling spinfluctuation theory and ask whether it provides a satisfactory description of the behavior of the self-energy, at least on a qualitative level, in the different regimes of temperature. This analysis will also provide guidance throughout the rest of this section in identifying which fluctuations contribute most to the self-energy.

In the simplest version of spin-fluctuation theory, the self-energy can be expressed as (omitting the Hartree term)

$$
\begin{aligned}
& \Sigma_{\mathrm{SF}}\left(\mathbf{k}, i \omega_{n}\right) \\
& \quad=g^{2} T \int \frac{d^{2} q}{(2 \pi)^{2}} \sum_{\Omega_{n}} G\left(\mathbf{k}+\mathbf{q}, i \omega_{n}+i \Omega_{n}\right) \chi\left(\mathbf{q}, i \Omega_{n}\right) .
\end{aligned}
$$

In this expression, $\chi\left(\mathbf{q}, i \Omega_{n}\right)$ is the momentum- and frequency-dependent spin susceptibility, and $G\left(\mathbf{k}, i \omega_{n}\right)$ is the (lattice) Green function. In the following, we always choose $G=G_{0}$ to be the noninteracting Green function; see the end of Sec. VII B for comments about the drawbacks of self-consistent schemes.

The coupling constant $g$ in Eq. (12) characterizes the coupling between electrons and the spin collective modes. It is renormalized as compared to its bare value as $U$ increases. In the following, we are more interested in asking whether weak-coupling spin-fluctuation theory qualitatively captures the different regimes than in quantitative statements. However, when a comparison is attempted, a value of $g$ has to be chosen. For example, in the version of 
TPSC considered in the present article, $g$ is given by (focusing on the spin channel only) $g_{\text {TPSC }}^{2}=\frac{3}{8} U U_{\text {sp }}$, with $U_{\text {sp }}=U\left\langle n_{\uparrow} n_{\downarrow}\right\rangle /\left\langle n_{\uparrow}\right\rangle\left\langle n_{\downarrow}\right\rangle$. The factor $3 / 8$ ensures rotational invariance by properly accounting for the relative contributions of longitudinal and transverse spin fluctuations [111], while the expression of $U_{\mathrm{sp}}$ is key to the TPSC scheme; see also Appendix D 11. In the following, we shall, for simplicity, use $g^{2}=3 U^{2} / 8$ when performing quantitative comparisons.

Figure 17 presents a comparison between the DГА results for the self-energy at the antinode $\mathbf{k}=(\pi, 0)$ and the spin-fluctuation expression Eq. (12) in which different choices are made for the spin susceptibility $\chi\left(\mathbf{q}, i \Omega_{n}\right)$. We perform the comparison using the susceptibility data from DГA due to its good agreement with the benchmark, the availability of the q-resolved susceptibility, and its ability to enter the highly insulating regime (5). This comparison is performed for three different temperatures, corresponding to the incoherent regime (1), the metallic regime (3), and the pseudogapped insulating regime (5).

We begin the discussion with the result obtained using the full (solid blue curve, square markers) DГA susceptibility. We see that, at a qualitative level, the spinfluctuation approximation succeeds in reproducing the characteristic low-frequency dependence of the self-energy associated with all three regimes. Furthermore, there is good quantitative agreement in the incoherent regime (1). With the chosen value of $g$, the self-energy is overestimated in both the metallic and pseudogap regimes. Using $g=g_{\text {TPSC }}$, together with the value of the double occupancy calculated in Fig. 12, largely remedies this overestimation for most of the metallic regime.

In order to better understand which spin fluctuations dominate the different regimes, we have also applied Eq. (12) by restricting the integration over the momentum transfer $\mathbf{q}$ to the vicinity of the antiferromagnetic wave vector $\mathbf{Q}=(\pi, \pi)$ according to $\left|q_{i}-Q_{i}\right|<2 \xi^{-1}, i \in\{x, y\}$ (dashed blue curves in Fig. 17). We see that in the pseudogap regime (5), the qualitative frequency dependence of the antinodal self-energy is not modified by this restriction (although obviously, as a result of the restriction in the integration, the self-energy is underestimated by an amount that is approximately frequency independent). Thus, in this regime, the self-energy is indeed dominated by the antiferromagnetic collective modes associated with the vicinity of $\mathbf{Q}=(\pi, \pi)$. In contrast, a completely different situation is found in the metallic regime (3): Restricting the momentum sum to the vicinity of $\mathbf{Q}$ yields a spinfluctuation self-energy that has a qualitatively different frequency dependence than the actual one, inconsistent with the properties of a metal (and actually closer to the shape associated with a pseudogap). It also underestimates the overall order of magnitude of the self-energy by more than a factor of 4 . Thus, we conclude that the single-particle properties in the metallic regime are not controlled only by the spin fluctuations associated with the antiferromagnetic wave vector: Taking into account spin fluctuations at other wave vectors is crucial. We will come back to this observation in Sec. VII C. Finally, we note that the incoherent regime is not affected by the momentum restriction simply because the correlation length is so small there that our criterion on the transfer momentum actually does not restrict the integration domain significantly.

Finally, we consider whether the spin susceptibility in Eq. (12) can be approximated, as often done in spinfluctuation theories, by an Ornstein-Zernike form emphasizing long-wavelength antiferromagnetic fluctuations:

$$
\chi\left(\mathbf{q}, i \Omega_{n}\right)=\frac{A}{(\mathbf{q}-\mathbf{Q})^{2}+\xi^{-2}+\frac{\left|\Omega_{n}\right|}{\gamma}} .
$$

As detailed in Appendix A, we use a lattice generalization of this expression and perform a fit of the momentum and frequency dependence of the DГА susceptibility in order to determine the amplitude $A$, correlation length $\xi$ (Sec. VI), and Landau damping coefficient $\gamma$ as a function of temperature. The antinodal self-energy obtained by inserting expression (13) into the spin-fluctuation expression (12) is displayed in Fig. 17 (purple data points). This approximation is quantitatively accurate in the high-temperature incoherent
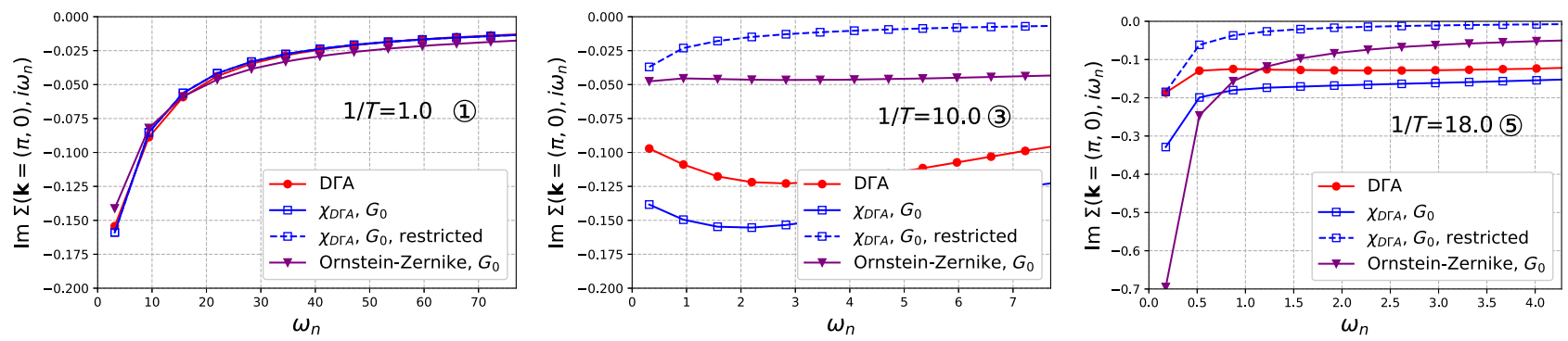

FIG. 17. Spin-fluctuation theory put to the test: comparison between the DГA self-energy and the result of weak-coupling spinfluctuation theory for three temperatures corresponding to regimes (1) (incoherent), (3) (metallic), and (5) (pseudogap). Both the full DГA susceptibility and its Ornstein-Zernike approximation are considered, as well as the effect of restricting the fluctuations to the longwavelength antiferromagnetic collective mode (see text). 
regime. It qualitatively captures the characteristic frequency dependence of a pseudogap in the low-temperature regime, as also detailed analytically in the following section, but it considerably overestimates its magnitude. In contrast, in the metallic regime, it fails quite severely both qualitatively and quantitatively, indicating that the Ornstein-Zernike approximation fails to capture important spectral weight in $\chi\left(\mathbf{q}, i \Omega_{n}\right)$ at $\mathbf{q}$ far from $(\pi, \pi)$, which is crucial in the metallic regime, as emphasized above and further detailed in Sec. VII C.

\section{B. Analytical insights into the pseudogap insulating regime}

Here, we provide some analytical insights into the low- $T$ pseudogap regime, based on the weak-coupling spinfluctuation theory and the Ornstein-Zernike approximation for the spin susceptibility in Eq. (13). As seen above, these approximations are qualitatively reasonable (although not quantitatively accurate) in this regime. For pioneering works on the formation of a pseudogap due to antiferromagnetic correlation, see Refs. [34,104]. In the latter, the authors focused on orientational fluctuations of the antiferromagnetic order described by a nonlinear sigma model and, expressing the physical electron as a product of a Schwinger boson and an auxiliary fermion, and were able to describe the crossover from a weak-coupling pseudogap in the Slater regime to a strong-coupling insulating gap in the Mott-Hubbard regime.

In this "renormalized classical" regime, only the lowest Matsubara frequency needs to be retained in Eq. (12), leading to $[34,89,94]$

$\operatorname{Im} \Sigma\left(\mathbf{k}_{\mathrm{F}}, i \omega_{n}\right)=-\tilde{g} \frac{T}{\sqrt{\omega_{n}^{2}-\omega_{c}^{2}}} \ln \frac{\omega_{n}+\sqrt{\omega_{n}^{2}-\omega_{c}^{2}}}{\omega_{n}-\sqrt{\omega_{n}^{2}-\omega_{c}^{2}}}$.

In this expression, the prefactor $A$ of the spin susceptibility has been combined with $g^{2}$ and numerical prefactors to yield a coupling $\tilde{g} \propto A g^{2}$ with the dimension of energy. In Eq. (14), only the dominant term has been retained; $\mathbf{k}_{\mathrm{F}}$ is a point on the Fermi surface away from the van Hove singularity (i.e., from the antinode), so the Fermi velocity $v_{F}$ is nonvanishing; and $\omega_{c}$ designates the important lowenergy scale:

$$
\omega_{c} \equiv \frac{v_{F}}{\xi}=\frac{v_{F}}{\xi_{0}} e^{-2 \pi \rho_{s} / T},
$$

which explicitly depends on the Fermi velocity (i.e., $\mathbf{k}_{\mathrm{F}}$ ). In the last expression, we postulated an exponential growth of the correlation length, characteristic of the low- $T$ gapped regime, with $\rho_{s}$ as the spin stiffness. Expression (14) has the following behavior at low and high (imaginary) frequencies:

$$
\operatorname{Im} \Sigma\left(\mathbf{k}_{\mathrm{F}}, i \omega_{n}\right) \sim-2 \tilde{g} T \frac{1}{\omega_{n}} \ln \frac{\omega_{n}}{\omega_{c}} \quad\left(\omega_{n} \gg \omega_{c}\right)
$$

$$
\sim-\pi \tilde{g} \frac{T}{\omega_{c}} \quad\left(\omega_{n} \ll \omega_{c}\right) .
$$

These expressions shed light on the dependence of the selfenergy on Matsubara frequency reported above in the low$T$ regime. The lowest Matsubara frequency in this regime is always larger than the tiny low-energy scale $\omega_{c}$ : In fact, the condition $\pi T \gg \omega_{c}$ defines the range of low temperatures in which the insulating or pseudogap behavior is observed $[34,94]$. In this regime, the Matsubara frequency selfenergy seemingly displays a downwards divergent behavior as in Eq. (16). However, if a calculation for low $\omega \lesssim \omega_{c}$ was possible, it would actually display a saturation to a finite value as in Eq. (17).

For completeness, we discuss the implications of this expression for the low-frequency spectral function and selfenergy on the real-frequency axis. Analytically continuing the above expression yields (for $\mathbf{k} \neq \mathbf{k}_{\mathrm{AN}}$ )

$$
\begin{gathered}
\operatorname{Re} \Sigma_{\mathrm{ret}}\left(\mathbf{k}_{\mathrm{F}}, \omega\right)=\tilde{g} \frac{T}{\sqrt{\omega^{2}+\omega_{c}^{2}}} \ln \left|\frac{\omega+\sqrt{\omega^{2}+\omega_{c}^{2}}}{\omega-\sqrt{\omega^{2}+\omega_{c}^{2}}}\right|, \\
\operatorname{Im} \Sigma_{\mathrm{ret}}\left(\mathbf{k}_{\mathrm{F}}, \omega\right)=-\pi \tilde{g} \frac{T}{\sqrt{\omega^{2}+\omega_{c}^{2}}},
\end{gathered}
$$

with the following asymptotic forms:

$$
\begin{aligned}
& |\omega| \gg \omega_{c}: \operatorname{Re} \Sigma \simeq 2 \frac{\tilde{g} T}{\omega} \ln \frac{\omega}{\omega_{c}}, \quad-\operatorname{Im} \Sigma \simeq \pi \frac{\tilde{g} T}{|\omega|}, \\
& |\omega| \ll \omega_{c}: \operatorname{Re} \Sigma \simeq 2 \frac{\tilde{g} T}{\omega_{c}^{2}} \omega, \quad-\operatorname{Im} \Sigma \simeq \pi \frac{\tilde{g} T}{\omega_{c}},
\end{aligned}
$$

with a slope obviously having a sign opposite to that of a Fermi liquid $(Z>1)$. The corresponding self-energy and spectral function on the Fermi surface $A(\mathbf{k}, \omega)=-\operatorname{Im}[\omega+$ $\left.\mu-\varepsilon_{\mathbf{k}}-\Sigma\right]^{-1} / \pi$ are plotted in Fig. 18. The two prominent

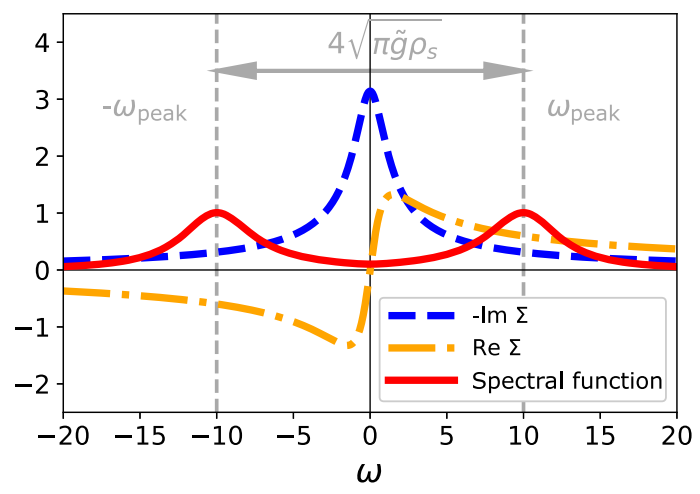

FIG. 18. Pseudogap regime: real (orange) and imaginary (blue) parts of the self-energy on the real axis phenomenologically calculated in weak-coupling spin-fluctuation theory and its corresponding spectral function (red). 
peaks at the edge of the pseudogap in the spectral function can be understood by using the asymptotic form of $\operatorname{Re} \Sigma$ for $\omega>\omega_{c}$ in the quasiparticle equation $\omega-\operatorname{Re} \Sigma(\omega)=0$. We see that the poles of the Green function are located at a frequency $\omega$ such that $\omega^{2}=2 \tilde{g} T \ln \omega / \omega_{c}$. The dominant term on the rhs of this equation is from $-2 \tilde{g} T \ln \omega_{c}$, and we finally obtain the locations of the main peaks at

$$
\omega_{\text {peak }}= \pm 2 \sqrt{\pi \tilde{g} \rho_{s}}+\cdots,
$$

where the corrections are linear in temperature. Hence, the location of these peaks becomes temperature independent in the low- $T$ limit. As $T \rightarrow 0$, these two peaks evolve continuously into the gap-edge peaks defining the singleparticle excitations across the insulating gap due to longrange antiferromagnetic order. Note that, within mean-field theory, the $T=0$ gap is given by $U m_{s}$, with $m_{s}$ the staggered magnetization, and that close to a quantum critical point, the scaling $m_{s} \propto \sqrt{\rho_{s}}$ indeed holds, establishing consistency with Eq. (22) (in the present case of $d=2$, additional logarithmic corrections intervene). Note that the above derivation of the pseudogap behavior down to $T=0$ relies on the exponential growth of the correlation length and hence would not apply if the ground state did not have long-range order. For an alternative approach based on thermal fluctuations starting from the $T=0$ ordered state, see Ref. [112].

The widths of the AF insulating peaks $[20,103,104]$ are of order $\left(\tilde{g} T^{2} / \rho_{s}\right)^{1 / 2}$ (for a DMFT analysis, see also Ref. [65]). Furthermore, it is interesting to understand the pseudogap regime in terms of lengths instead of the energy $\omega_{c}$ [34]. The one-particle thermal de Broglie wavelength $v_{F} /(\pi T)$ determines the maximum distance over which an electron wave packet remains coherent despite thermal agitation. Equation (21) shows that the imaginary part of the self-energy at zero frequency becomes very large when this length is smaller than the correlation length for spin fluctuations, leading to the decrease of spectral weight at zero frequency. For electrons in the pseudogap regime, the spins appear to be ordered over the length where temperature does not destroy its coherence. This argument holds only in two dimensions. In higher dimensions, the phase space associated with the integration over wave vectors in Eq. (12) diminishes the importance of long-wavelength fluctuations [34] for the self-energy. As is well known from DMFT, in infinite dimensions, the self-energy is not influenced by longwavelength spin fluctuations $[9,10]$.

A similar analysis can be performed for Fermi-surface points in the vicinity of a van Hove singularity, i.e., when $v_{F}=0$. The expansion of the dispersion relation is then stopped only after the second-order term, giving, for $\mathbf{k}_{\mathrm{AN}}=$ $(\pi, 0)$ after integration over $q_{x}$,

$$
\begin{aligned}
& -\frac{1}{\pi} \operatorname{Im} \Sigma_{\mathrm{ret}}\left(\mathbf{k}_{\mathrm{AN}}, \omega\right)= \\
& \frac{\tilde{g}^{2}}{(2 \pi)^{2}} T \int d q_{y} \frac{1}{\sqrt{\omega+q_{y}^{2}}} \frac{1}{\omega+\xi^{-2}+2 q_{y}^{2}},
\end{aligned}
$$

which yields logarithmic divergences. Even if these divergences could be cut off, for $\omega \rightarrow 0$,

$$
-\frac{1}{\pi} \operatorname{Im} \Sigma_{\mathrm{ret}}\left(\mathbf{k}_{\mathrm{AN}}, \omega \rightarrow 0\right) \propto \frac{1}{2} \tilde{g}^{2} T \xi^{2},
$$

so the imaginary part of $\Sigma$ is now proportional to $\xi^{2}$ instead of $\xi$ (see also Refs. [34,51,113], [114,115] for second-order perturbation theory (2PT) and Ref. [116] in the context of ferromagnetic fluctuations), i.e., way larger than for momenta away from van Hove points.

To conclude this subsection on the pseudogap insulating regime, we recall some findings and comment on the ability of the description of this regime in self-consistent theories. First, we note that if one uses $G$ (with a finite self-energy) instead of $G_{0}$ as a fermionic propagator in Eq. (12), one obtains $\operatorname{Im} \Sigma(\mathbf{k}, 0) \propto T^{-2}$, hence missing the exponential growth of Eqs. (17) and (25) [51] (see also Ref. [103]).

On more general grounds, we note that the selfconsistency at the level of vertex corrections should be consistent with the one of the Green function. This remark serves as a warning about imposing self-consistency solely over the Green function. When using approximate schemes that truncate perturbation theory or select a specific class of diagrams without taking vertex corrections into account in a consistent manner, self-consistency may lead to an incorrect physical description-especially in relation to the opening of insulating gaps. For an insightful discussion of these limitations of self-consistent perturbation theory, see Sec. 6.1 of Ref. [34]. Observations about the inadequacy of self-consistency in truncated perturbation theory approximations have previously been made in other contexts as well, such as the GW approximation and DMFT, where it is well known that self-consistent perturbation theory truncated to second order fails in yielding Hubbard bands and the opening of a Mott gap [10,117-120]. However, an additional, completely different problem can arise when self-consistent theories (e.g., bold diagrammatic Monte Carlo [121,122]) are applied: Because of the multivaluedness of the Luttinger-Ward functional [123-127], intimately related [128] to divergences in vertex functions [129-136], the bold approaches may converge to an unphysical branch.

\section{Insights into the metallic regime}

\section{Temperature dependence and characteristic scales}

Finally, in this section, we provide insights into the physics of the metallic regime. In Fig. 19, we display the quantities 

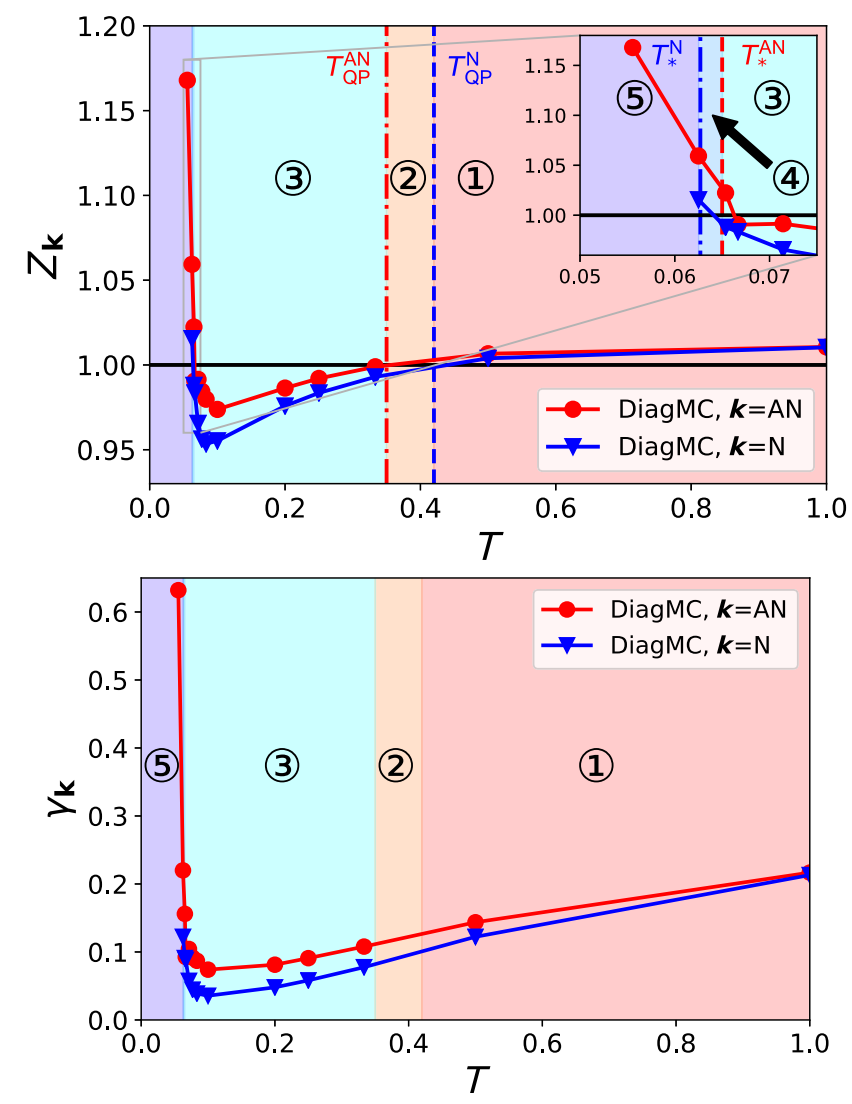

FIG. 19. Quasiparticle parameters $Z_{\mathbf{k}}$ (upper panel; the gray box denotes the boundaries of the inset) and $\gamma_{\mathbf{k}}$ (lower panel) at the antinode (red circles) and node (blue triangles) for the DiagMC benchmark as a function of temperature.

$$
Z_{\mathbf{k}}=\left[1-\left.\frac{\partial \operatorname{Im} \Sigma(\mathbf{k}, i \omega)}{\partial \omega}\right|_{\omega \rightarrow 0}\right]^{-1}
$$

and

$$
\gamma_{\mathbf{k}}=\tau_{\mathbf{k}}^{-1}=-\left.Z_{\mathbf{k}} \cdot \operatorname{Im} \Sigma(\mathbf{k}, i \omega)\right|_{\omega \rightarrow 0}
$$

as a function of temperature, at the nodal and antinodal points on the Fermi surface, obtained by a fit to the DiagMC self-energy on Matsubara frequencies. For the details about how these parameters are extracted, see Appendix B. For a Fermi liquid, $Z_{\mathbf{k}}$ would correspond to the quasiparticle spectral weight and $\gamma_{\mathbf{k}}$ to the inverse of the quasiparticle lifetime. However, we are dealing with a perfectly nested system in which Fermi-liquid behavior does not strictly apply, and this interpretation has to be taken with care, as further discussed in Sec. VII C 3. Indeed, we observe that $\gamma_{\mathbf{k}}$ at the nodal point follows an approximately $T$-linear temperature dependence for $T \gtrsim 0.1$. The antinodal value of $\gamma_{\mathbf{k}}$ is significantly larger, highlighting momentum differentiation.

The metallic regime (3) was conventionally defined above as the regime where the self-energy has a negative slope at low frequency, thus allowing us to define a $Z_{\mathbf{k}}$ smaller than unity, as indicated on the upper panel of Fig. 19. From the $T$ dependence of $\gamma_{\mathbf{k}}$, we see that regime (3) defined in this manner actually consists of two subregimes (see also Refs. [137,138]): For $0.1 \lesssim T \lesssim T_{\mathrm{QP}}^{\mathrm{AN}}$ (regime 3a covering most of region (3), the inverse quasiparticle lifetime $\gamma_{\mathbf{k}}$ decreases upon reducing $T$, indicating an increase of quasiparticle coherence upon cooling (as is characteristic of a metal). When cooling below $T \simeq 1 / 10$, a minimum of $\gamma_{\mathbf{k}}$ (and $Z_{\mathbf{k}}$ ) is found, below which the lifetime decreases upon cooling as a precursor of the pseudogap regime (regime (3b).

In order to rationalize these findings, we turn again to spinfluctuation theory, Eq. (12). In contrast to the pseudogap regime, it is crucial here to perform the full convolution over all Matsubara frequencies. Using the spectral representation of $\chi\left(\mathbf{q}, i \Omega_{n}\right)$, the single-particle scattering rate at zero frequency is obtained from Eq. (12) as

$$
\begin{aligned}
& -\operatorname{Im} \Sigma\left(\mathbf{k}, i 0^{+}\right) \\
& \sim g^{2} \int d^{2} q \int d \omega \frac{1}{\sinh (\beta \omega)} \operatorname{Im} \chi(\mathbf{q}, \omega) \delta\left(\omega-\varepsilon_{\mathbf{k}+\mathbf{q}}\right) .
\end{aligned}
$$

The key point here is to compare the width in frequency of the two factors entering this expression: $1 / \sinh \beta \omega$ and $\operatorname{Im} \chi(\mathbf{q}, \omega)$. The width of the former is of order temperature $T$, while the width of the latter is set by Landau damping, as is clear from the Ornstein-Zernike form Eq. (13) applicable close to the antiferromagnetic wave vector, which reads, for real frequencies,

$$
\operatorname{Im} \chi(\mathbf{q}, \omega)=A \frac{\omega / \gamma}{\left[(\mathbf{q}-\mathbf{Q})^{2}+\xi^{-2}\right]^{2}+(\omega / \gamma)^{2}} .
$$

This expression peaks at the characteristic spin-fluctuation frequency:

$$
\omega_{\mathrm{sf}}=\gamma \xi^{-2} .
$$

Hence, for $T \lesssim \omega_{\text {sf }}$, only the low-frequency behavior of $\operatorname{Im} \chi(\mathbf{q}, \omega)$ matters in Eq. (28). This analysis shows that, besides the scale $\omega_{c}=v_{F} / \xi$ that controls the pseudogap regime, the spin-fluctuation scale $\omega_{\mathrm{sf}}=\gamma \xi^{-2}$ is important for the physics of the metallic regime (see Ref. [139] and references therein). In Fig. 20, we display these two scales as a function of temperature, with $\xi(T)$ and $\gamma(T)$ determined from a fit to the ВГА susceptibility as described above and in Appendix A. We compare these two scales to the temperature itself and observe that this indeed defines three distinct regimes, which correspond to a very good approximation to the regimes observed in our numerical results, namely (note that $\left.\omega_{\text {sf }}<\omega_{c}\right)$,

(i) $T \lesssim \omega_{\text {sf }}(\xi \lesssim \sqrt{\gamma / T})$ : metallic regime (3)a.

(ii) $T \gtrsim \omega_{c} \quad\left(\xi \gtrsim v_{F} / T\right.$, Vilk criterion): insulating pseudogap regime (4), (5). 


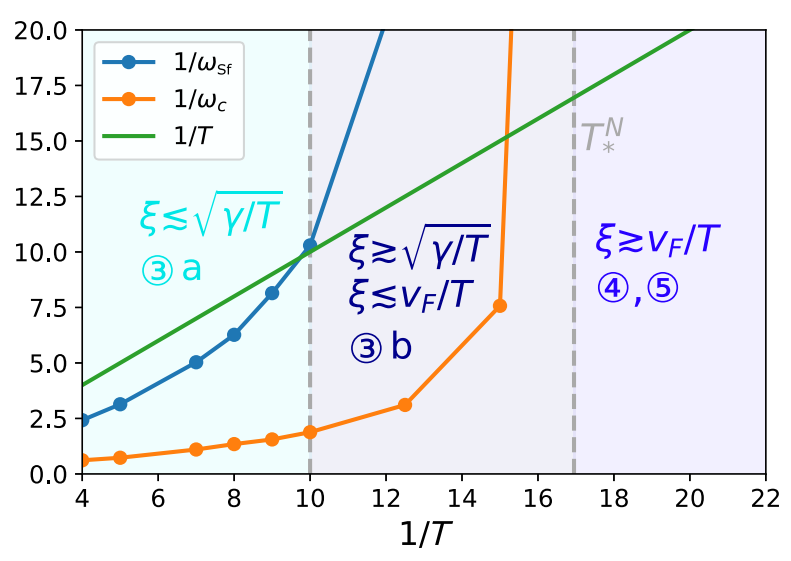

FIG. 20. Comparison of the two energy scales $\omega_{c}=v_{F} / \xi$ and $\omega_{\text {sf }}=\gamma \xi^{-2}$ to inverse temperature (or, equivalently, of the correlation length to the two length scales $\sqrt{\gamma / T}$ and $v_{F} / T$ ). This comparison delimitates three regimes: the metallic regime (3)a, a narrow precursor regime of the pseudogap (3b, and a nonmetallic pseudogap regime (4), (5). The scales are determined by a fit to the frequency and momentum dependence of the DГA susceptibility (see text).

(iii) $\omega_{\text {sf }} \lesssim T \lesssim \omega_{c}\left(v_{F} / T \gtrsim \xi \gtrsim \sqrt{\gamma / T}\right)$. In this narrow intermediate regime $(0.06 \lesssim T \lesssim 0.1$, (3b), the pseudogap is not yet fully opened, but the scattering rate no longer behaves as in a metal: It displays a minimum and increases at lower $T$ as the pseudogap regime is entered, as displayed in Fig. 19. We note, in passing, that the upper boundary of this regime coincides with the temperature at which the double occupancy displays a local maximum (see Fig. 12), corresponding to the onset temperature above which local magnetic moments are formed (see also Refs. [49,136]). It also has corresponding signatures in other thermodynamic observables [49].

\section{Which fluctuations dominate the metallic regime?}

Expression (28) also allows us to understand an important observation made in Sec. VII A, namely, that in the metallic regime, the spin fluctuations contributing to the self-energy are not dominated only by the vicinity of the antiferromagnetic wave vector.

In order to further document and validate this point, in Fig. 21, we display a comparison between the momentum dependence of the spin susceptibility as obtained in DГА and that of its Ornstein-Zernike fit (the latter privileges the vicinity of the antiferromagnetic wave vector). We see (left panel) that at $T=1 / 8$, which lies in the metallic regime, the OZ fit very poorly captures the momentum dependence of the susceptibility far from $\mathbf{Q}$. In particular, the OZ fit misses the sizable weight that is visible in the DГA susceptibility along the diagonals $\left(q_{x}, \pm q_{x}\right)$. That weight comes from scattering associated with nesting vectors parallel to the antiferromagnetic zone boundaries, as is apparent already in second-order perturbation theory [114]. In contrast, the $\mathrm{OZ}$ form is much more accurate at lower
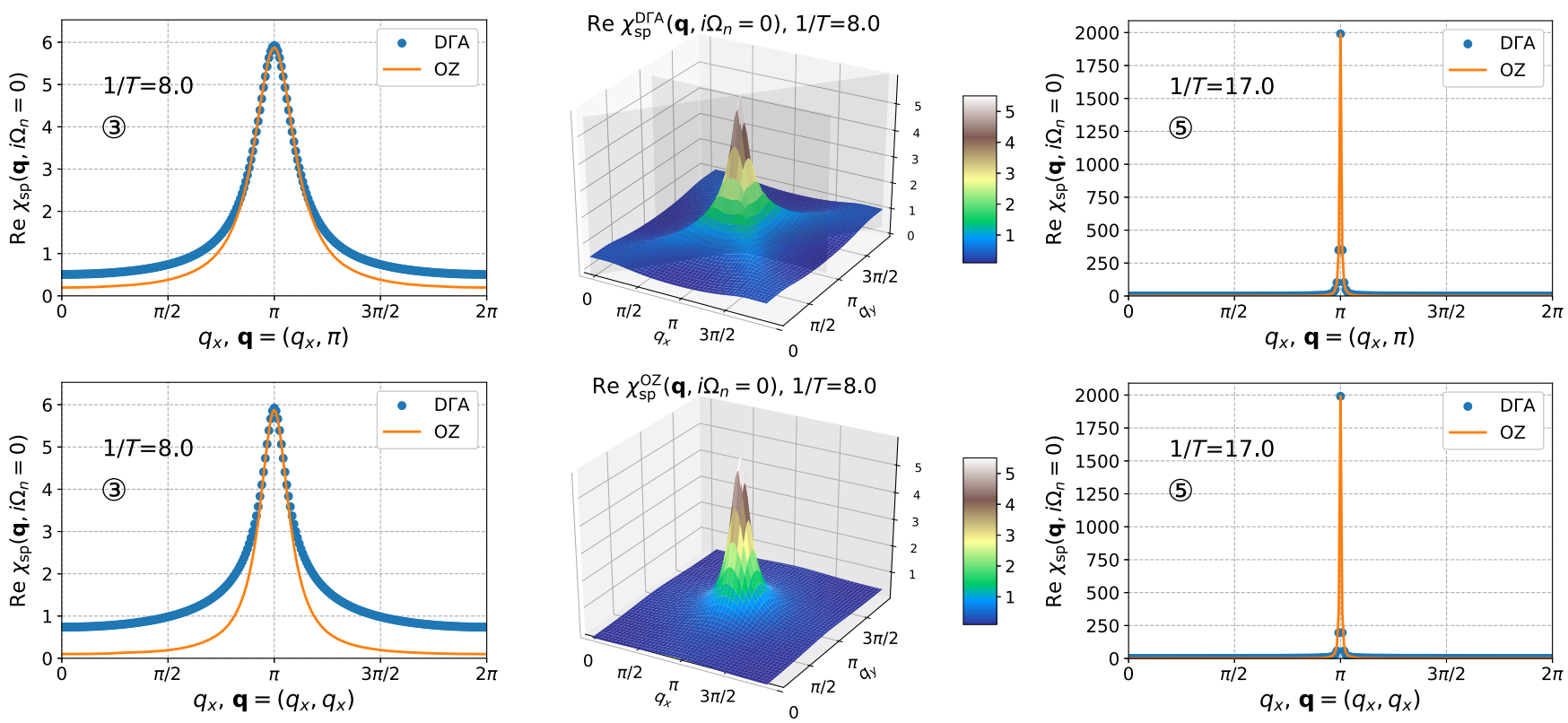

FIG. 21. Comparison between the static antiferromagnetic susceptibilities obtained from DГA and fits to an Ornstein-Zernike form (see Appendix A and Figs. 25 and 27 for the corresponding fit parameters). In the temperature regime around $1 / T=8$, the DГA susceptibility has, in addition to the antiferromagnetic peak at $\mathbf{Q}=(\pi, \pi)$, important contributions associated with the nesting vectors of the form $\left(q_{x}, \pm q_{x}\right)$ ("ridges" in the upper middle panel). Although describing the vicinity of $\mathbf{Q}=(\pi, \pi)$ accurately, the Ornstein-Zernike form does not account for these contributions (lower middle panel). At lower temperatures, these contributions become negligible. The gray planes in the middle upper panel indicate the cuts shown, i.e., $\mathbf{q}=\left(q_{x}, \pi\right)$ and $\mathbf{q}=\left(q_{x}, q_{x}\right)$. 


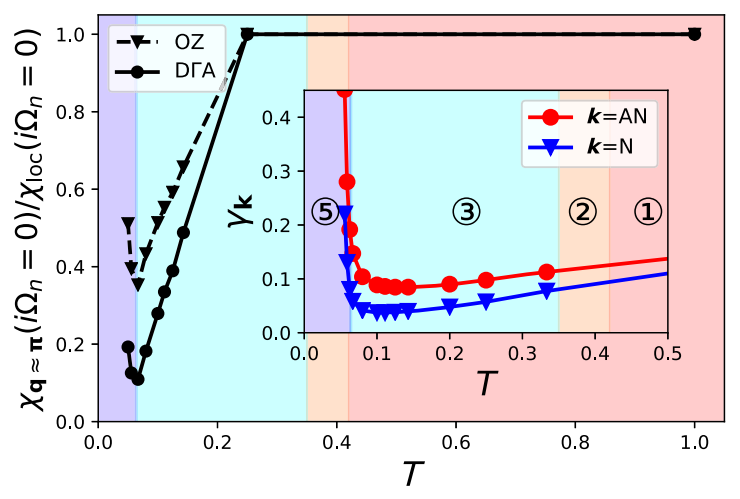

FIG. 22. Main panel: relative contributions to the AF susceptibility from wave vectors in the vicinity of $\mathbf{Q}=(\pi, \pi)$ for DГA (solid line, black circles) and the Ornstein-Zernike fit (dashed line, black triangles) as a function of $T$. Inset: For completeness, $\gamma_{\mathbf{k}}$ from DГА is shown for the different regimes as a function of temperature for the antinode (red circles) and node (blue triangles).

temperatures when $\xi$ has grown to larger values and the system is in the pseudogap regime (right panel): Indeed, the physics of the pseudogap is dominated by antiferromagnetic fluctuations (Sec. VII B).

This argument is further documented in Fig. 22, which displays the ratio between the susceptibility integrated over a region of the order of $2 \xi^{-1}$ around $\mathbf{Q}$ to its integral over the whole zone. It is seen that this ratio decreases as $T$ is lowered throughout the metallic regime, while it increases again in the pseudogap regime: Hence, a large fraction of the spectral weight is missed by focusing on the vicinity of $\mathbf{Q}$ in the metallic regime. Figure 22 also shows that the $\mathrm{OZ}$ fit overestimates the relative spectral weight associated with the vicinity of the antiferromagnetic wave vector.

Using the expressions (28) and (29), it can be shown that the contribution of the $(\pi, \pi)$ spin fluctuations to the zero-frequency scattering rate on the FS (away from the van Hove point) behaves in the metallic regime as $\sim g^{2}(A / \gamma) T^{2} \xi^{3} / v_{F}$. As shown in Appendix A, the ratio $A / \gamma$ decreases quickly upon cooling in this regime, which ensures that this contribution to the scattering rate also decreases despite the fast increase of the correlation length. A similar remark applies to the local (momentumintegrated) scattering rate, for which this contribution can be estimated as $\sim g^{2} \rho_{0}(A / \gamma) T^{2} \xi^{2}$ (up to logarithmic terms). However, importantly, the local self-energy involves contributions from fluctuations at all wave vectors, as is already clear at the level of the spin-fluctuation approximation by integrating Eq. (28) over k. This explains why DMFT provides, in the metallic regime, an excellent approximation to the local component of the selfenergy. In contrast, the DMFT approximation does not properly capture the contributions to the self-energy of fluctuations that are strongly peaked at a specific wave vector. For a plot of the (negative) imaginary part of the local self-energy extrapolated to zero Matsubara frequency as a function of temperature, we refer to Fig. 28 in Appendix A.

Summarizing, we have shown that a comparison of the two key energy scales $\omega_{c}=v_{F} / \xi$ and $\omega_{\text {sf }}=\gamma \xi^{-2}$ to temperature allows for a determination of the different physical regimes as a function of temperature, in excellent quantitative agreement with our numerical results. The momentum-differentiated metallic regime evolves at low $T$ into a precursor regime of the pseudogap, where the quasiparticle lifetime reaches a maximum. We have also shown that the metallic regime is not dominated by the spin fluctuations associated with only the antiferromagnetic wave vector, and we explained why quasiparticles with a lifetime increasing upon cooling can coexist with antiferromagnetic fluctuations characterized by a correlation length that strongly increases (approximately exponentially).

\section{Perfect nesting and non-Fermi-liquid behavior}

In the half-filled model with $t^{\prime}=0$ considered in this paper, we do not expect Fermi-liquid behavior to hold in the metallic phase because of perfect nesting. Indeed, the whole one-dimensional set of wave vectors with $q_{x}= \pm q_{y}$ maps a point on the diamond-shaped Fermi surface onto another one. In this section, we briefly discuss whether our computations can detect hints of non-Fermi-liquid behavior, despite providing data restricted to imaginary (Matsubara) frequencies.

In a Fermi liquid, the self-energy takes the following form, when considered on the Fermi surface and at low frequency and temperature:

$$
-\operatorname{Im} \Sigma\left(\mathbf{k}_{\mathrm{F}}, \omega\right) \propto \omega^{2}+(\pi T)^{2}+\cdots .
$$

On the Matsubara axis, the rhs of this expression reads $-\omega_{n}^{2}+(\pi T)^{2}$. This contribution vanishes when considering the first Matsubara frequency $\omega_{0}=\pi T$. Hence, the temperature dependence of $\operatorname{Im} \Sigma\left(\mathbf{k}_{\mathrm{F}}, i \omega_{0}\right)$ at the first Matsubara frequency is controlled by the dominant term $(1-1 / Z) \omega_{0}=$ $(1-1 / Z) \pi T+\cdots$, with no quadratic correction of order $T^{2}$ : Linear dependence of this quantity on temperature is a hallmark of Fermi-liquid behavior (first Matsubara frequency rule; see Ref. [140]). Figure 23 displays the $T$ dependence of this quantity as obtained from the benchmark DiagMC at both the nodal and antinodal points on the Fermi surface. We see a visible deviation from linearity throughout the metallic regime, indeed hinting at a departure from Fermiliquid behavior. Further evidence for non-Fermi-liquid behavior is provided by the dependence on temperature of the parameters $Z_{\mathbf{k}}$ and $\gamma_{\mathbf{k}}$ obtained by a fit of the self-energy on Matsubara frequencies and displayed in Fig. 19. Indeed, (i) $Z_{\mathbf{k}}$ does not stabilize to a $T$-independent value before the pseudogap kicks in but rather slowly decreases throughout the metallic regime, and (ii) the temperature dependence of $\gamma_{\mathbf{k}}$ is better described as $T$ linear rather than quadratic-like in 


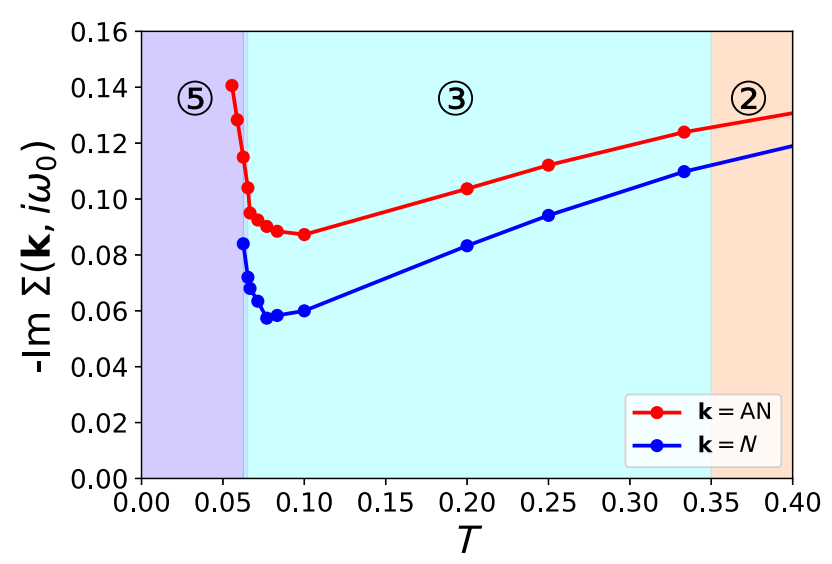

FIG. 23. Diagnosing non-Fermi-liquid behavior: The imaginary part of the self-energy at the first Matsubara frequency calculated by DiagMC for the nodal and antinodal points displays the deviation from linearity as a function of $T$ (see text).

a Fermi liquid. Further details about the proper interpretation of the Matsubara fitted parameters $Z_{\mathbf{k}}$ and $\gamma_{\mathbf{k}}$ when Fermiliquid behavior does not apply are given in Appendixes B and $\mathrm{C}$.

The breakdown of Fermi-liquid theory in a perfectly nested system was explored earlier in Ref. [141] and subsequent works. To understand its origin, it is useful to consider the spin-fluctuation expression (28) and note that, when $\operatorname{Im} \chi$ is free from singularities and behaves linearly as a function of $\omega$ at low frequency, this equation yields the characteristic Fermi-liquid $T^{2}$ behavior for $T \lesssim \omega_{\text {sf }}$ and $\omega=0$ :

$$
-\operatorname{Im} \Sigma\left(\mathbf{k}, i 0^{+}\right) \sim g^{2} T^{2} \int d^{2} q \frac{1}{\Gamma_{\mathbf{q}}} \delta\left(\varepsilon_{\mathbf{k}+\mathbf{q}}\right),
$$

in which $1 / \Gamma_{\mathbf{q}} \equiv \operatorname{Im} \chi(\mathbf{q}, \omega) /\left.\omega\right|_{\omega=0}$. In turn, non-Fermiliquid behavior in a perfectly nested system can be traced to singularities in $\operatorname{Im} \chi(\mathbf{q}, \omega)$. These singularities are already manifest at the level of second-order perturbation theory, as studied in detail in Ref. [114] and also recently in Ref. [137]. In Appendix C, we discuss secondorder perturbation theory in more detail, together with a comparison to our computational results. Theoretical approaches of the non-Fermi-liquid singularities for nested systems beyond lowest-order perturbation theory have been attempted using parquet [142] and fRG $[143,144]$ methods, but we are not aware of a full solution of the case considered in the present paper.

From a computational standpoint, a precise characterization of non-Fermi-liquid behavior in the metallic phase would require algorithms that are able to determine the behavior of the self-energy and response functions directly on the real-frequency axis, in both the $\omega>T$ and $\omega<T$ regimes (the latter being inaccessible to methods that provide data for Matsubara frequencies only). This task goes beyond the scope of the present work and is a major challenge motivating the development of new computational methods (see, e.g., Refs. [145-148]).

\section{A simple approximation}

In the previous sections, we have seen that fluctuations from all momentum transfers $\mathbf{q}$ contribute to the local (momentum-integrated) part of the self-energy in the metallic regime. Long-wavelength antiferromagnetic fluctuations associated with $\mathbf{q} \simeq \mathbf{Q}$ become increasingly important as the temperature is lowered and are responsible for the formation of the pseudogap. This observation suggests trying a simple approximation in which one relies on DMFT for the local part of the self-energy, while a weakcoupling approximation, Eq. (12), is used to account for the nonlocal part, namely,

$\Sigma\left(\mathbf{k}, i \omega_{n}\right) \simeq \Sigma_{\mathrm{DMFT}}\left(i \omega_{n}\right)+\left[\Sigma_{\mathrm{SF}}\left(\mathbf{k}, i \omega_{n}\right)-\Sigma_{\mathrm{SF}}^{\mathrm{loc}}\left(i \omega_{n}\right)\right]$.

In this expression, the last term removes the local contribution from the spin-fluctuation formula, so the local, $\mathbf{k}$-averaged part of the self-energy is entirely accounted for by DMFT. Note that, in this subsection, we take the selfenergy from paramagnetically restricted DMFT. To evaluate Eq. (33), we use the bare value $g^{2}=3 U^{2} / 8$ of the coupling to spin fluctuations and the ВГA spin susceptibility. We recall that the latter is simply given by $\chi^{-1}(\mathbf{q}, \omega)=$ $\chi_{\text {DMFT }}^{-1}(\mathbf{q}, \omega)+\lambda$, with $\lambda$ the Moriya correction, which ensures consistency with the Mermin-Wagner theorem and is determined such that $T \sum_{\mathbf{q}, n} \chi\left(\mathbf{q}, i \Omega_{n}\right)=\chi_{\text {loc }}$, with $\chi_{\text {loc }}$ being the local susceptibility as obtained in DMFT from the effective impurity model (for details, see Appendix D 6 a). Expression (33) is actually a simplified version of the DГA, in which vertex functions are set to unity. (See also Appendix D 6 a for the DГA equation of motion. For an analysis in the context of superconductivity in cuprates, see Ref. [149].)

The result of this simple "DMFT+SF" approximation of Eq. (33) is compared, in Fig. 24 (top row), to the DiagMC benchmark and to DГA for three temperatures. We see that it performs excellently in the incoherent and in the metallic regime. In the pseudogap regime, it is qualitatively reasonable, but it overestimates the magnitude of the selfenergy at low frequency (in accordance with the observations made above.) This case indicates that, for this rather weak value of the coupling $U=2$, the vertex correction terms included in DГА are not essential in the metallic regime but become increasingly important as the temperature is lowered into the pseudogap regime.

The lower panels in Fig. 24 display the local part of the self-energy as obtained from DMFT, together with its approximation from weak-coupling spin-fluctuation theory [the local term removed in Eq. (33)], in comparison to DiagMC. This result clearly demonstrates that a weakcoupling spin-fluctuation approximation does not provide a good estimate of the local component, even at this weak 

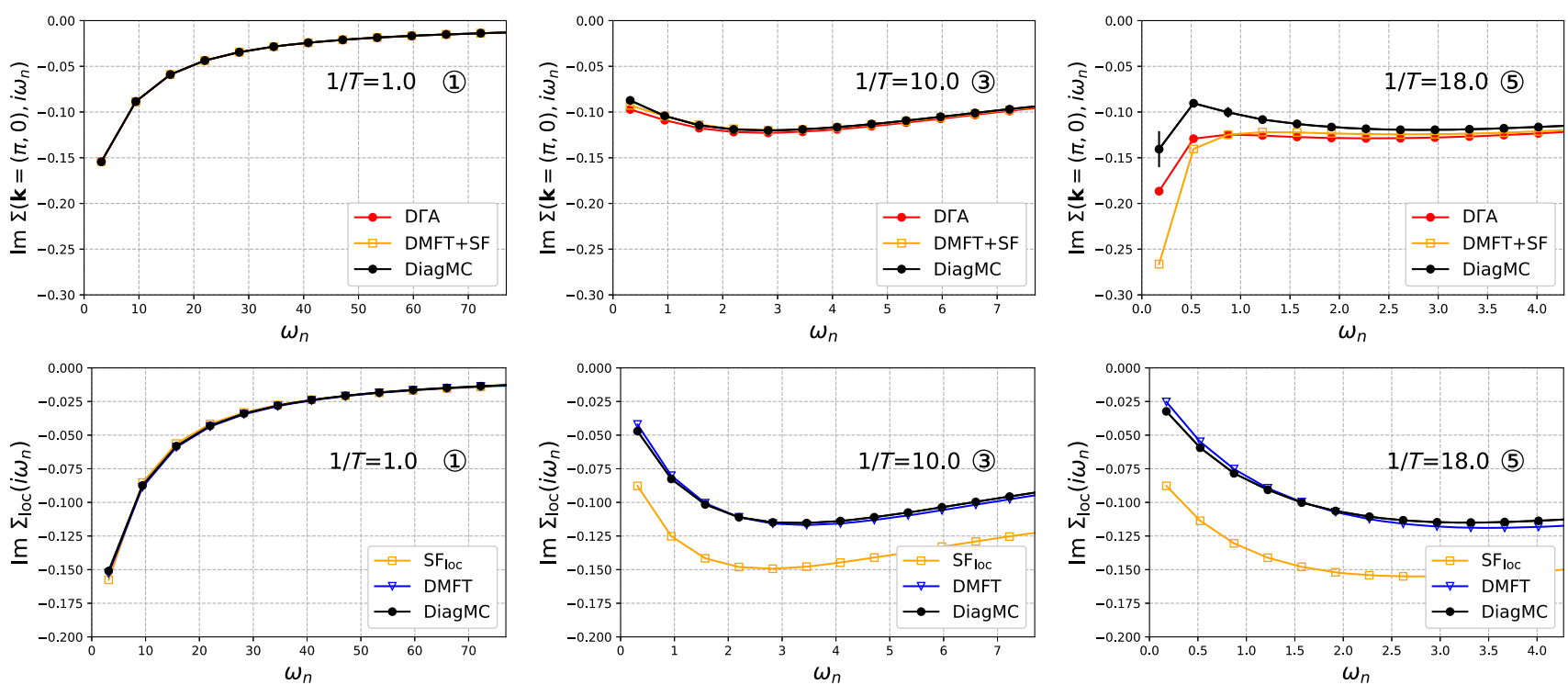

FIG. 24. Upper row: weak-coupling spin-fluctuation theory combined with DMFT according to Eq. (33)—a simplified version of $\mathrm{D} Г \mathrm{~A}$ ignoring vertex corrections (see text) and comparison to the DiagMC benchmark (black circles). Lower row: comparison between the local self-energy as obtained from DMFT and the corresponding weak-coupling spin-fluctuation approximation to the DiagMC benchmark (black circles).

value of $U$. In contrast, the nonperturbative DMFT provides an excellent description of the $\mathbf{k}$-averaged (local) self-energy, while nonlocal contributions can be reasonably accounted for by weak-coupling spin-fluctuation theory at this value of $U$.

\section{CONCLUSION AND OUTLOOK}

In this article, we have purposefully focused on an apparently "simple" regime of the two-dimensional Hubbard model (simple square lattice, weak coupling, half-filling) with three goals in mind: (i) assessing the ability of state-of-the-art computational methods to address the physics of this model at finite temperature, through the computation of a range of one-particle and two-particle observables; (ii) providing an extensive assessment of basically all many-body methods currently available for this purpose, by comparing them to two very different Monte Carlo methods (DQMC and DiagMC) serving as benchmarks; and, importantly, (iii) investigating the rich physics associated with the different regimes and crossovers found in this model, as it evolves upon cooling from a high-temperature incoherent regime into a momentumdifferentiated metal and eventually into an insulating regime with a pseudogap, and elucidating the nature and role of spin fluctuations in these different regimes.

It is satisfying to observe that the two benchmark methods considered in this article are successful at computing the properties of this model throughout the metallic regime and are in excellent agreement with each other. However, we also found that they face rather severe limitations when attempting to reach low temperatures, to the extent that only the onset of the pseudogap regime can be reached. Indeed, the pseudogap is associated with an exponentially growing antiferromagnetic correlation length, which requires prohibitively large systems to be simulated with DQMC, while DiagMC, which, in contrast, works directly in the thermodynamic limit, is faced with convergence issues when summing the perturbative series at low $T$. We are hopeful that these issues can be overcome by using recent improvements of diagrammatic Monte Carlo based on the CDet algorithm [25], together with improved schemes for summing the perturbative series.

Many of the methods considered in this article use the DMFT as a starting point and treat spatial fluctuations beyond this starting point to various degrees of approximation. Obviously, the chosen regime of parameters puts DMFT very much out of its "comfort zone" since the physics at low $T$ is dominated by long-range spatial fluctuations, which, in two dimensions, also prevent longrange order at any nonzero temperature. Nonetheless, we have emphasized and documented that, when properly interpreted, DMFT provides a useful initial description of the different regimes. Indeed, in the low- $T$ pseudogap regime when the correlation length is large, describing the system as long-range ordered is a reasonable mean-field starting point. Furthermore, remarkably, we found that DMFT provides a highly accurate determination of the local (momentum-averaged) self-energy, not only at high $T$, as expected, but also through most of the metallic regime. This observation is striking given that the correlation length reaches values as large as ten lattice spacings in this regime, and we further comment below on the physical reason explaining this finding. 
Because of the very large correlation length at low $T$, cluster extensions of DMFT are not the best route to follow in this parameter regime of the Hubbard model. Although they succeed in describing the momentum-differentiated metallic regime in satisfactory agreement with the benchmarks, huge cluster sizes would be required to properly capture the pseudogap regime. Cluster extensions of DMFT are much better equipped for addressing strong-coupling regimes with shorter correlation lengths.

In contrast, we found that extensions of DMFT (ladderDГA, TRILEX, ladder-DF, and single-shot DB), which make use of response and vertex functions in order to take into account the contributions of fluctuations to the selfenergy, perform much better here. We assessed their respective degree of accuracy and found that the DГА and DF/DB methods are particularly successful at describing all physical regimes of interest, in satisfactory agreement with the benchmarks, including the onset of the pseudogap regime. They also allow one to enter deeper in the pseudogap regime: It is an open question for future work to assess their degree of validity in this low- $T$ regime when accurate and controlled benchmarks become available.

We have also considered many-body methods that do not make use of DMFT as a starting point, such as TPSC/ TPSC + , fRG, and the PA. TPSC played an important role early on in elucidating the physical origin of the weakcoupling pseudogap in relation to long-wavelength antiferromagnetic correlations. We found that it captures the different regimes qualitatively but strongly overestimates the onset of the pseudogap temperature and the correlation length itself. TPSC + , a recent extension of the method, leads to significant improvements. In contrast, the PA underestimates the pseudogap onset temperature, especially at the nodal point, whereas it captures the behavior of double occupancy very accurately. The conventional one-loop fRG shown here is in qualitative agreement with the benchmark until its running coupling constants diverge. However, we remark that the present implementation can be systematically improved by the recently introduced multiloop extension of fRG that converges to the parquet approximation [57,150], as well as expanding around the DMFT solution $\left(\mathrm{DMF}^{2} \mathrm{RG}\right)[151,152]$. Their combination, a multiloop expansion of the fRG around DMFT, provides a very promising direction for future studies.

On the physics side, perhaps the most intriguing finding of our study is that, in the metallic regime, single-particle excitations with a lifetime that increases upon cooling appear to happily coexist with an antiferromagnetic correlation length that increases steeply, reaching about ten lattice spacings at the onset of the pseudogap. We have provided an explanation to this finding and also shown that spin fluctuations associated with the vicinity of the antiferromagnetic wave vector are not the only important contributions to the self-energy in this regime. Fluctuations from all nesting vectors also contribute to the self-energy on the Fermi surface, and fluctuations from all momentum transfers contribute especially to the local, momentum-integrated, self-energy. Importantly, this observation explains why the latter is so accurately captured by single-site DMFT, even in the presence of strong antiferromagnetic fluctuations, which are responsible for the momentum differentiation on the Fermi surface.

At the lowest temperatures, antiferromagnetic fluctuations eventually dominate, destroying the coherent metal, which gives way to the pseudogap regime (with a narrow precursor regime at which the quasiparticle lifetime reaches a maximum). This result provides a rationale for the success of methods such as DГA and the DF, which incorporate fluctuations of all wave vectors beyond DMFT. In the parameter regime of interest, we have proposed and tested a simplified version of such an approach.

We have documented, in detail, the rich sequence of crossovers occurring in this model upon reducing the temperature, from an incoherent regime at high $T$ all the way down to the pseudogap regime at low $T$, through the intermediate-temperature momentum-differentiated metal, and we have assessed the ability of the different computational methods to capture these crossovers. By analyzing the contribution of spin fluctuations, we showed that these different regimes are delimited by the comparison of temperature itself to the two characteristic energy scales $v_{F} / \xi$ and $\gamma \xi^{-2}$ or, equivalently, of the correlation length $\xi$ to the thermal de Broglie wavelength, $\sim v_{F} / T$, and to the length scale associated with Landau damping, $\sim \sqrt{\gamma / T}$ (with $\gamma$ being the Landau damping constant).

Finally, we have also emphasized (Sec. VII C 3 and Appendixes B and C) the limitations of imaginary-time (Matsubara frequency) computational methods in probing the delicate, low-energy, non-Fermi-liquid singularities of this perfectly nested model in the metallic regime.

In the future, the numerical, theoretical, and physical insights obtained in this article should find direct applications in computational studies $[149,153,154]$ of materials for which the interplay between local electronic correlations and long-wavelength spin fluctuations play a crucial role, such as $\mathrm{Sr}_{2} \mathrm{RuO}_{4}$ [155-157] or the iron-based superconductors [158]. On the model level, our findings will be immediately useful for describing how physical fluctuations (density, magnetic, and pairing) on the two-particle level influence one-particle spectral functions and self-energies, and how quasiparticles are affected and sometimes destroyed altogether by these fluctuations [128,159-164].

\section{ACKNOWLEDGMENTS}

We would like to thank Andrey Chubukov, Chloé GauvinNdiaye, Emanuel Gull, Karsten Held, Carsten Honerkamp, Andrey A. Katanin, Alexander I. Lichtenstein, Walter Metzner, Andrew J. Millis, Alice Moutenet, Georg Rohringer, Subir Sachdev, Michael Thoennessen, and 
Alessandro Toschi for useful and valuable discussions, as well as T. S. Barry for support and encouragement. T. S. and A. G. are indebted to Katharina Kölbl and Sandrine Kott for their infinite patience during the writing phase of this manuscript. The present work was supported by the following: the Austrian Science Fund (FWF) through the ErwinSchrödinger Fellowship J 4266, "Superconductivity in the vicinity of Mott insulators" (SuMo, T. S.), and Projects No. P 30997 (C. E.) and No. P 30819 (A. K.); the European Research Council for the European Union Seventh Framework Program (FP7/2007-2013) with ERC Grant No. 319286 (QMAC, T.S. and A. G.); the Simons Foundation through the Simons Collaboration on the Many Electron Problem (M.F., F.Š., and E. K.); the Natural Sciences and Engineering Research Council (Canada) under Grants No. RGPIN-2019-05312 (A.-M. S. T.) and No. RGPIN-2017-04253 (J. P. F. L.); and the Canada First Research Excellence Fund, the Research Chair on the Theory of Quantum Materials, Compute Canada and Calcul Québec (Y.W. and A.-M. S. T.). A. J. K. and E. K. acknowledge funding from EPSRC through Grant No. EP/P003052/1. V.H. and E. A. S. acknowledge support from the NorthGerman Supercomputing Alliance (HLRN) under Project No. hhp00042. C.H. and S. A. acknowledge financial support from the Deutsche Forschungsgemeinschaft (DFG) through Projects No. AN 815/4-1 and No. AN 815/6-1. T. S., F.S. ., and M. F. acknowledge the hospitality of the Center for Computational Quantum Physics at the Flatiron Institute. The Flatiron Institute is a division of the Simons Foundation. The authors acknowledge the computer support teams at CPHT École Polytechnique, CEA Saclay (IPHT-LSCE), and the Flatiron Institute, as well as the computer service facility of the MPI-FKF for their help. The authors were granted access to the HPC resources of TGCC and IDRIS under the allocations A0070510609 and A0090510609 attributed by GENCI (Grand Equipement National de Calcul Intensif). Parts of the simulations were performed on computers provided by Calcul Québec and Compute Canada. J.P.F. L. acknowledges computational support from ACENET and Compute Canada. The PA calculations were performed on the Vienna Scientific Cluster (VSC) and JURECA at Forschungszentrum Jülich [165]. D. R. gratefully acknowledges the computing time granted through JARA on the supercomputer JURECA [165] at Forschungszentrum Jülich.

\section{APPENDIX A: ORNSTEIN-ZERNIKE FORM OF THE MAGNETIC SUSCEPTIBILITY}

Close to a magnetic phase transition, the magnetic susceptibility (bosonic propagator) assumes the following Ornstein-Zernike form [110]:

$$
\chi\left(\mathbf{q}, i \Omega_{n}\right)=\frac{A}{(\mathbf{q}-\mathbf{Q})^{2}+\xi^{-2}+\frac{\left|\Omega_{n}\right|}{\gamma}},
$$

with $A$ being a prefactor, $\gamma$ the Landau damping constant (the dynamical critical exponent has been set to $z=2$ here), and $\xi$ the magnetic correlation length. This form can be used to evaluate the spin-fluctuation diagram given in Eq. (12). In the renormalized classical regime (5), the sum may be restricted to the lowest Matsubara frequency without great loss of precision, which is, however, not possible in the metallic regime (3). The estimation of the correlation length from the static magnetic susceptibility in this paper has been performed by an empirically more robust formula (especially in the limit of small $\xi$ ) of the Ornstein-Zernike form, which incorporates the lattice (tight-binding) structure of the problem [72], neglecting a possible anomalous exponent $\eta$ (cf. $\eta \approx 0.037$ for the three-dimensional Heisenberg model [166]):

$$
\chi_{\mathrm{sp}}\left(\mathbf{q}, i \Omega_{n}=0\right)=\frac{A}{4 \sin ^{2}\left(\frac{q_{x}-Q_{x}}{2}\right)+4 \sin ^{2}\left(\frac{q_{y}-Q_{y}}{2}\right)+\xi^{-2}},
$$

and reduces to the original form in the long-wavelength limit (small arguments of the sine). For the fits shown, we fixed $q_{y}=\pi$. Figure 25 shows data for the static antiferromagnetic susceptibility in ВГА (upper row) and the respective Ornstein-Zernike fits for various temperatures. Note, again, as discussed in the main text, that the OrnsteinZernike fit is most accurate only around the vicinity of $\mathbf{Q}=(\pi, \pi)$ and may not capture the physics at other $\mathbf{q}$ points, which is important, e.g., in the metallic regime (see Sec. VII C). These fits of the static antiferromagnetic susceptibility are used to determine the prefactor $A$ and the magnetic correlation length $\xi$. For extracting the Landau damping $\gamma, A$ and $\xi$ have been fixed, and $\gamma$ has been fitted from the frequency dependence of the propagator. The upper panels of Fig. 26 show these fits of ВГА data for three representative temperatures. The lower panels show the obtained parameters $A, \xi$, and $\gamma$ as a function of temperature.

We see that the prefactor $A(T)$ rapidly decreases with temperature in the metallic regime. This can be rationalized by observing that the local susceptibility $\chi_{\text {loc }}=\int d^{2} q /(2 \pi)^{2} \chi\left(\mathbf{q}, \Omega_{n}=0\right)$ obeys a Curie-Weiss law in this regime, implying that $A \ln \xi \sim C /(T+\Theta)$ and hence that $A$ decreases linearly with $T$. In contrast, in the low- $T$ insulating regime, the sum rule $T \sum_{n} \int d^{2} q /(2 \pi)^{2} \chi\left(\mathbf{q}, \Omega_{n}=0\right)=n-2\left\langle n_{\uparrow} n_{\downarrow}\right\rangle \quad$ implies $T A \ln \xi \sim$ const, and hence, $A$ reaches a constant value at low $T$. For the temperature dependence of the local spin susceptibility in DГA, see Fig. 27. The (negative) imaginary part of the local self-energy extrapolated to zero Matsubara frequency as a function of temperature, relevant for the discussion in Sec. VII C 2, is shown in Fig. 28. 


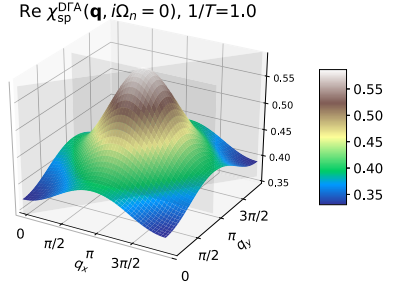

$\operatorname{Re} \chi_{\mathrm{sp}}^{\mathrm{oZ}}\left(\mathbf{q}, i \Omega_{n}=0\right), 1 / T=1.0$
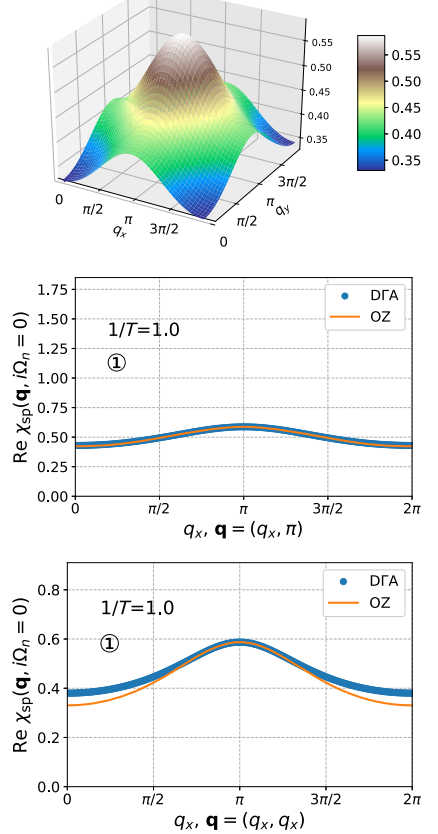

$\operatorname{Re} \chi_{\mathrm{sp}}^{\mathrm{D}}\left(\mathbf{q}, i \Omega_{n}=0\right), 1 / T=4.0$

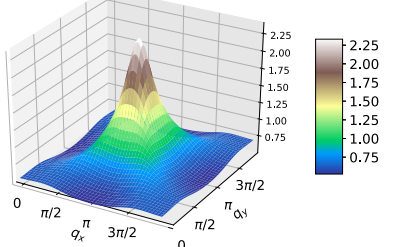

$\operatorname{Re} \chi_{\mathrm{sp}}^{\mathrm{oZ}}\left(\mathbf{q}, i \Omega_{n}=0\right), 1 / T=4.0$
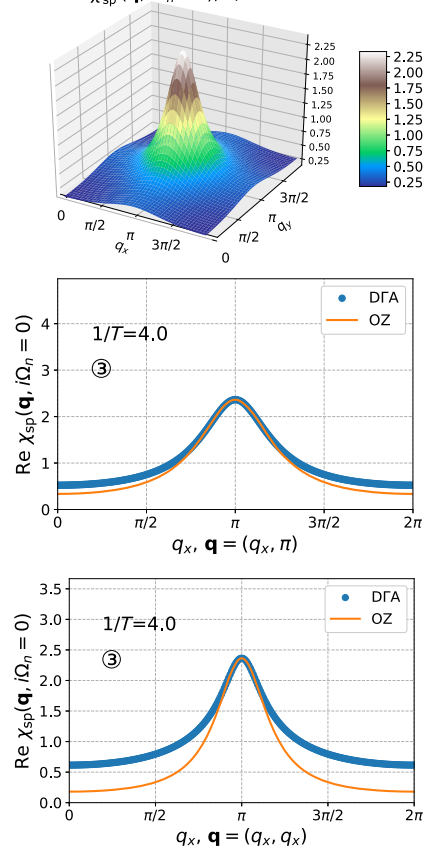

$\operatorname{Re} \chi_{s p}^{\mathrm{D} \Gamma \mathrm{A}}\left(\mathbf{q}, i \Omega_{n}=0\right), 1 / T=12.5$

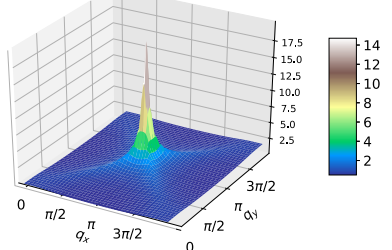

$\operatorname{Re} \chi_{\mathrm{sp}}^{\mathrm{OZ}}\left(\mathbf{q}, i \Omega_{n}=0\right), 1 / T=12.5$

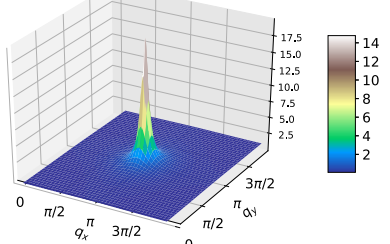

$\operatorname{Re} \chi_{\mathrm{sp}}^{\mathrm{D} \Gamma \mathrm{A}}\left(\mathbf{q}, i \Omega_{n}=0\right), 1 / T=15.0$

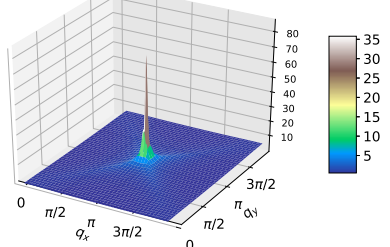

$\operatorname{Re} \chi_{\mathrm{sp}}^{\mathrm{OZ}}\left(\mathbf{q}, i \Omega_{n}=0\right), 1 / T=15,0$

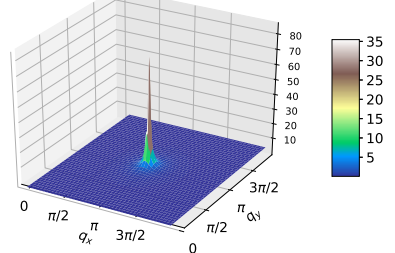

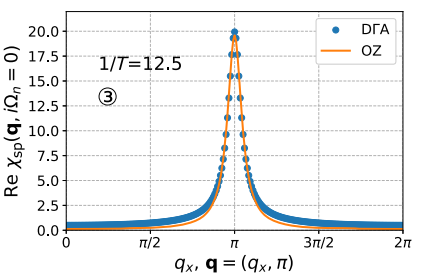
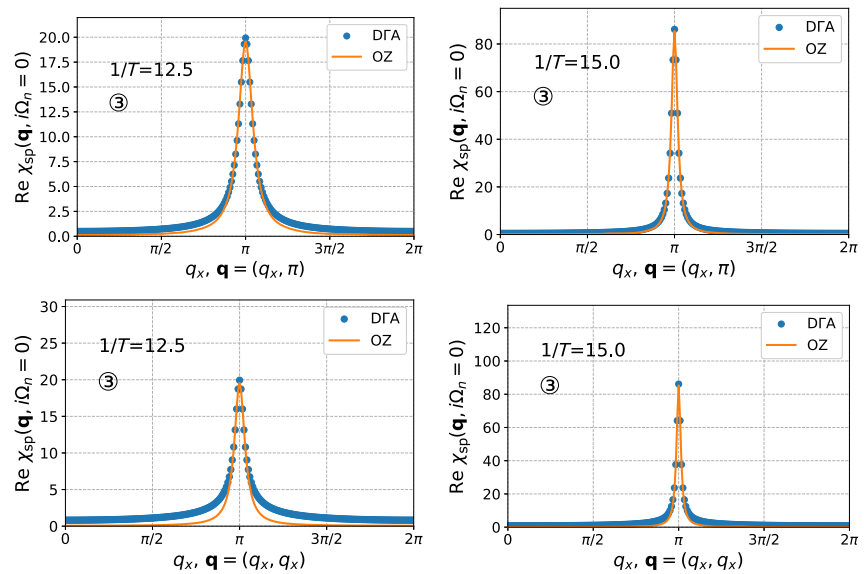

FIG. 25. Static (zero-frequency) antiferromagnetic susceptibilities obtained from DГА (uppermost row), the fitted data from an Ornstein-Zernike form (second row), and a comparison of both at the momentum cuts $\mathbf{q}=\left(q_{x}, \pi\right)$ (third row) and $\mathbf{q}=\left(q_{x}, q_{x}\right)$ (lowest row) for various temperatures.
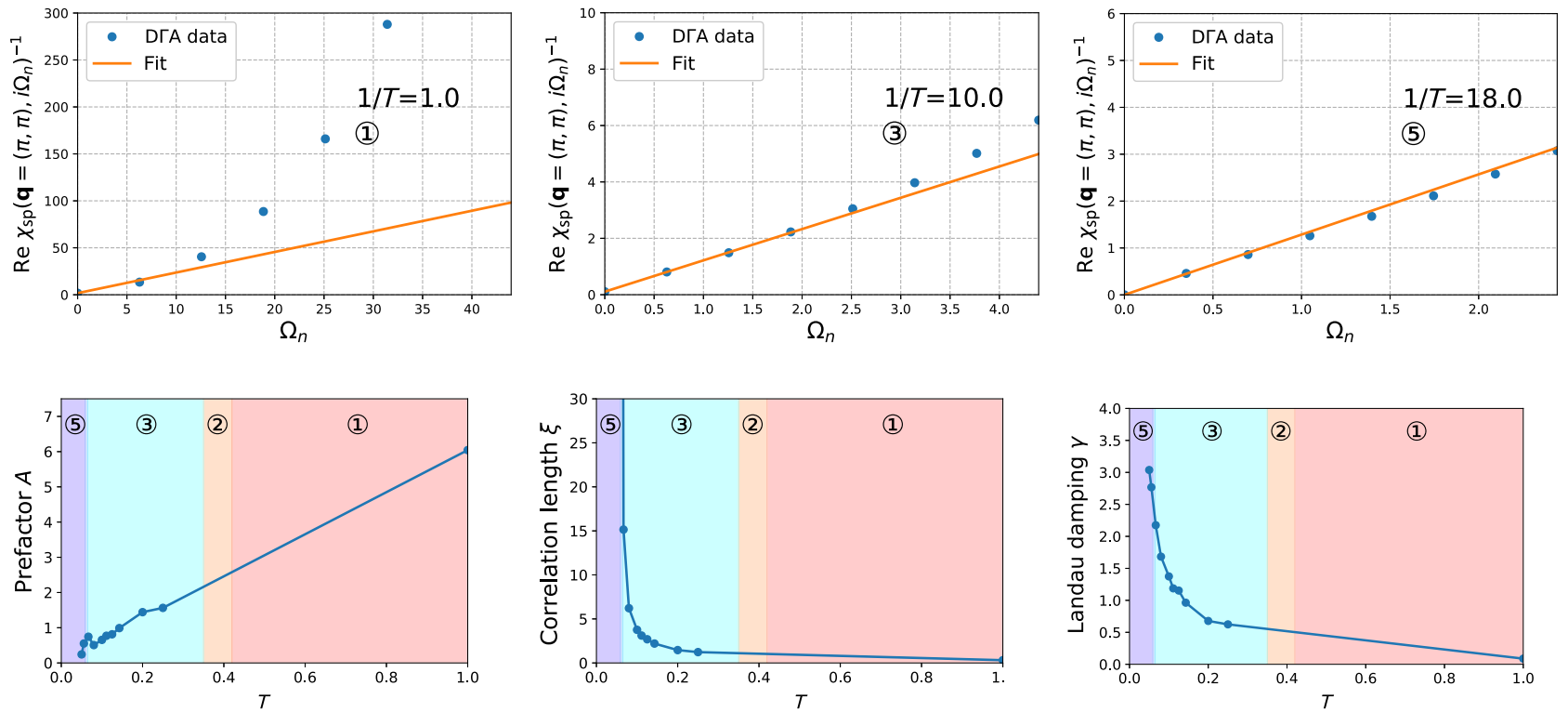

FIG. 26. Upper row: Fits of the frequency-dependent part of the (inverse) antiferromagnetic susceptibility from DГA by an OrnsteinZernike form at three dierent temperatures. Lower row: The prefactor A and the magnetic correlation length $\xi$ have been fixed to the ones of the static propagator and their temperature dependencies together with the one of the Landau damping $\gamma$ are shown. 


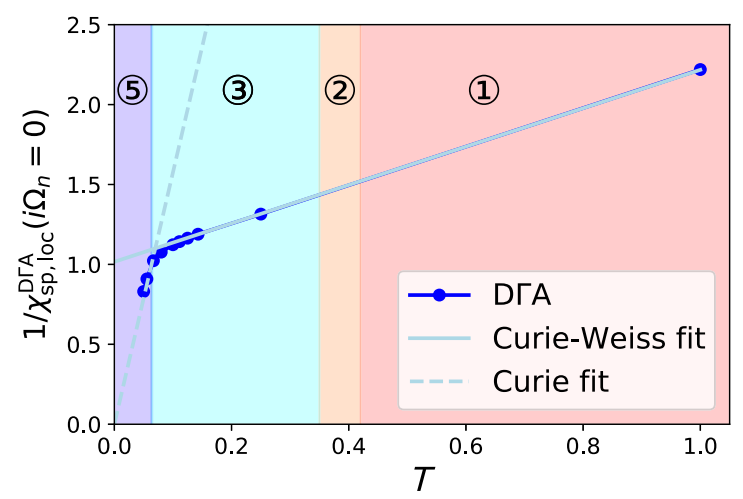

FIG. 27. Inverse local spin susceptibility calculated in DГA as a function of temperature, which exhibits Curie-Weiss (Curie) behavior in the metallic (insulating) regime.

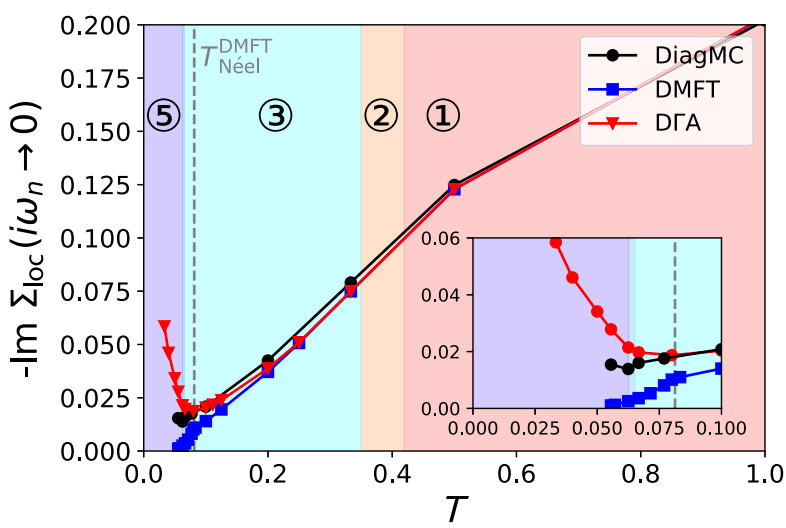

FIG. 28. Imaginary (negative) part of the local self-energy extrapolated to zero Matsubara frequency as a function of temperature calculated in DiagMC (black circles), DMFT (blue squares), and DГA (red triangles).

\section{APPENDIX B: LOW-FREQUENCY EXPANSION OF THE SELF-ENERGY}

In a Fermi liquid, the self-energy on the Fermi surface can be expanded at low (imaginary) frequency as a Taylor series:

$$
\begin{aligned}
\Sigma\left(\mathbf{k}_{\mathrm{F}}, i \omega \rightarrow 0\right) \simeq & \operatorname{Re} \Sigma\left(\mathbf{k}_{\mathrm{F}}, 0\right)+i \operatorname{Im} \Sigma\left(\mathbf{k}_{\mathrm{F}}, 0\right) \\
& +i \omega \frac{\partial \operatorname{Im} \Sigma\left(\mathbf{k}_{\mathrm{F}}, i \omega\right)}{\partial \omega}+\cdots
\end{aligned}
$$

Inserting this expression into Dyson's equation $G^{-1}=$ $G_{0}^{-1}-\Sigma$, we see that the spectral function displays a quasiparticle peak with a spectral weight $Z_{\mathbf{k}}$ and width $\gamma_{\mathbf{k}}$ (inverse of the quasiparticle lifetime $\tau_{\mathbf{k}}$ ) given by

$$
\begin{gathered}
Z_{\mathbf{k}}=\left[1-\left.\frac{\partial \operatorname{Im} \Sigma(\mathbf{k}, i \omega)}{\partial \omega}\right|_{\omega \rightarrow 0}\right]^{-1}, \\
\gamma_{\mathbf{k}}=\tau_{\mathbf{k}}^{-1}=-\left.Z_{\mathbf{k}} \cdot \operatorname{Im} \Sigma(\mathbf{k}, i \omega)\right|_{\omega \rightarrow 0} .
\end{gathered}
$$

Performing, also, a Taylor expansion for momenta $\mathbf{k}$ close to the Fermi surface, the renormalization of the quasiparticle effective mass is obtained as

$$
\left.\left(\frac{m^{*}}{m}\right)^{-1}\right|_{\mathbf{k}_{F}}=Z_{\mathbf{k}_{F}}\left[1+\frac{\hat{\mathbf{e}}_{\mathbf{k}_{F}} \cdot \nabla_{k} \operatorname{Re} \Sigma(\mathbf{k}, i \omega \rightarrow 0)}{\hat{\mathbf{e}}_{\mathbf{k}_{F}} \cdot \nabla_{k} \varepsilon_{\mathbf{k}}}\right]_{\mathbf{k}=\mathbf{k}_{F}} .
$$

In practice, $Z_{\mathbf{k}}$ and $\gamma_{\mathbf{k}}$, as displayed, e.g., in our discussion of the metallic regime in Fig. 19, are obtained in this article by a fit of the imaginary part of the self-energy $\operatorname{Im} \Sigma\left(\mathbf{k}, i \omega_{n}\right)$ calculated on the discrete set of Matsubara frequencies, by a fourth-order polynomial. This method is common practice in analyzing computational results available in imaginary time at finite temperature. The sign of the slope of the self-energy at low Matsubara frequencies was also used to distinguish between "metallic" behavior $\left(Z_{\mathbf{k}}<1\right)$ and the pseudogap and incoherent regimes in which the positive slope corresponds formally to $Z_{\mathbf{k}}>1$. Care should be applied, however, in interpreting the values of $Z_{\mathbf{k}}$ and $\gamma_{\mathbf{k}}$ obtained from such Matsubara fits in terms of quasiparticle properties, when the system does not obey Fermi-liquid behavior at low frequencies or low temperatures (as is the case here because of perfect nesting and of the gradual opening of the pseudogap). We illustrate and clarify this point by considering, for simplicity, a linear fit of the imaginary part of the self-energy over the first two Matsubara frequencies $\omega_{0}=\pi T$ and $\omega_{1}=3 \pi T$, namely,

$\operatorname{Im} \Sigma\left(i \omega_{n}\right)=\left.\operatorname{Im} \Sigma\left(i 0^{+}\right)\right|_{\text {linfit }}+\omega_{n}\left(1-\frac{1}{Z_{\text {linfit }}}\right)+\cdots$,

with

$$
\begin{aligned}
\left.\operatorname{Im} \Sigma\left(i 0^{+}\right)\right|_{\text {linfit }} & =\frac{\omega_{1} \operatorname{Im} \Sigma\left(i \omega_{0}\right)-\omega_{0} \operatorname{Im} \Sigma\left(i \omega_{1}\right)}{\omega_{1}-\omega_{0}}, \\
1-\frac{1}{Z_{\text {linfit }}} & =\frac{\operatorname{Im} \Sigma\left(i \omega_{1}\right)-\operatorname{Im} \Sigma\left(i \omega_{0}\right)}{\omega_{1}-\omega_{0}} .
\end{aligned}
$$

For simplicity, we have dropped the momentum dependence in these expressions: It is understood that the analysis is performed at a given value of $\mathbf{k}$. We now use the spectral representation of the self-energy $\Sigma\left(i \omega_{n}\right)=$ $\int d \omega\left[\sigma(\omega) / i \omega_{n}-\omega\right]$, with $\sigma(\omega) \equiv-(1 / \pi) \operatorname{Im} \Sigma\left(\omega+i 0^{+}\right)$, yielding

$$
-\operatorname{Im} \Sigma\left(i \omega_{n}\right)=\omega_{n} \int d \omega \frac{\sigma(\omega)}{\omega_{n}^{2}+\omega^{2}}
$$

Substituting this into Eqs. (B6), after simple algebra, we finally obtain 


$$
\begin{gathered}
-\left.\operatorname{Im} \Sigma\left(i 0^{+}\right)\right|_{\operatorname{linfit}}=12 \pi^{3} T^{3} \int d \omega \frac{\sigma(\omega)}{\left(\pi^{2} T^{2}+\omega^{2}\right)\left(9 \pi^{2} T^{2}+\omega^{2}\right)}, \\
1-\frac{1}{Z_{\text {linfit }}}=\int d \omega \sigma(\omega) \frac{3 \pi^{2} T^{2}-\omega^{2}}{\left(\pi^{2} T^{2}+\omega^{2}\right)\left(9 \pi^{2} T^{2}+\omega^{2}\right)} .
\end{gathered}
$$

We now analyze how these two quantities behave in the lowtemperature limit. To this aim, we consider cases in which $\omega / T$ scaling applies for small $\omega$ and $T$ (but an arbitrary ratio $\omega / T)$, and where $\sigma(\omega, T)=T^{\alpha} \phi(\omega / T)$, with $\phi$ a scaling function such that $\phi(x \ll 1) \sim$ const and $\phi(x \gg 1) \sim|x|^{\alpha}$. The case $\alpha=2$ corresponds to a Fermi liquid for which the scaling function is known to be $\phi(x)=A\left(\pi^{2}+x^{2}\right)$. A value of $\alpha<2$ corresponds to non-Fermi-liquid behavior with $\omega / T$ scaling, as found, for example, close to quantum critical points controlled by strong-coupling fixed points [167]. For an analysis of non-Fermi-liquid behavior and $\omega / T$ scaling within second-order perturbation theory, see Appendix C.

To obtain the low- $T$ behavior of $-\left.\operatorname{Im} \Sigma\left(i 0^{+}\right)\right|_{\text {linfit }}$, we observe that the $\omega / T$ scaling form can be directly substituted in the above expression while preserving the convergence of the integral, which leads to, for $T \rightarrow 0$,

$$
-\left.\operatorname{Im} \Sigma\left(i 0^{+}\right)\right|_{\text {linfit }} \sim 12 \pi^{3} T^{\alpha} \int d x \frac{\phi(x)}{\left(\pi^{2}+x^{2}\right)\left(9 \pi^{2}+x^{2}\right)} .
$$

This expression demonstrates that a fit to the self-energy on Matsubara frequencies is able to correctly capture the nonFermi-liquid temperature dependence of the scattering rate. Note that for this to hold, it is crucial that $\omega / T$ scaling indeed applies. This derivation has been performed, for simplicity, for a linear fit, but it extends to a higher-order polynomial fit.

The low- $T$ analysis of $Z_{\text {linfit }}$ proceeds along slightly different lines. Indeed, direct substitution of the $\omega / T$ scaling form into the equation above would lead to an ultraviolet divergent integral when $\alpha>1$. In that case, the limit $T \rightarrow 0$ can directly be taken to yield

$\alpha>1:\left.Z_{\text {linfit }}\right|_{T \rightarrow 0}=\left[1+\int d \omega \frac{\sigma(\omega, T=0)}{\omega^{2}}\right]^{-1}$.

This formula is indeed the exact $T=0$ expression of the quasiparticle weight resulting from the spectral representation. Note that, for $\sigma(\omega) \sim \omega^{\alpha}$ with $\alpha>1$ at low frequency, the above integral converges in the infrared and that, in this case, there are coherent quasiparticles, with a finite spectral weight and a scattering rate $\sim T^{\alpha}$ smaller than their energy. In the opposite case $\alpha<1$, the $\omega / T$ scaling function must be used, which yields the low- $T$ behavior:

$$
Z_{\text {linfit }} \sim\left[1+\frac{1}{T^{1-\alpha}} \times \int d x \phi(x) \frac{x^{2}-3 \pi^{2}}{\left(\pi^{2}+x^{2}\right)\left(9 \pi^{2}+x^{2}\right)}\right]^{-1} .
$$

At low $T, Z_{\text {linfit }}$ vanishes at $T^{1-\alpha}$, indicating the breakdown of the quasiparticle concept. For $\alpha=1$, a very slow logarithmic vanishing of $Z$ is expected.

In conclusion, this analysis demonstrates that when $\omega / T$ scaling applies, together with a simple power-law behavior, a fit to the self-energy over Matsubara frequencies is able to pick up the correct $T$ dependence of the scattering rate and quasiparticle weight in both the Fermi-liquid and nonFermi-liquid cases, in spite of the fact that the frequency dependence of the self-energy for $\omega<T$ is inaccessible from Matsubara frequencies. Note that $\omega / T$ scaling typically applies in the quantum critical regime associated with quantum critical points (QCP) controlled by a strongcoupling fixed point [167]. In this work, the QCP is the one associated with the disappearance of antiferromagnetism at $U=0$, and hence, it is not clear whether $\omega / T$ scaling applies in the metallic regime. Logarithmic violations may be expected, for example.

\section{APPENDIX C: SELF-ENERGY FROM SECOND-ORDER PERTURBATION THEORY}

For further reference, and to facilitate the discussion of the consequences of perfect nesting in Sec. VII C 3, here we show the results of $2 \mathrm{PT}$ for the self-energy.

\section{Real frequencies}

We first consider the self-energy in 2PT on the realfrequency axis (see also Refs. [114,137]). Its functional form can be gained by using the careful analytic calculations performed in Sec. 4.4 of Ref. [114], which we summarize in this subsection. We start by considering the expression on the Matsubara axis,

$\operatorname{Im} \Sigma\left(\mathbf{k}, i \omega_{n}\right)=U^{2} T \sum_{\mathbf{q}, i \Omega_{n}} G_{0}\left(\mathbf{k}+\mathbf{q}, i \omega_{n}+i \Omega_{n}\right) \chi_{0}\left(\mathbf{q}, i \Omega_{n}\right)$,

with the (noninteracting) bubble

$\chi_{0}\left(\mathbf{q}, i \Omega_{n}\right)=-T \sum_{\mathbf{k}^{\prime}, i \omega_{n^{\prime}}} G_{0}\left(\mathbf{k}^{\prime}+\mathbf{q}, i \omega_{n^{\prime}}+i \Omega_{n}\right) G_{0}\left(\mathbf{k}^{\prime}, i \omega_{n^{\prime}}\right)$,

and $G_{0}$ being the noninteracting Green function.

\section{a. Analytical considerations}

Analytic continuation of Eq. (C1) gives

$$
\begin{aligned}
\operatorname{Re} \Sigma(\mathbf{k}, \omega)= & \frac{U^{2}}{8 \pi^{3}} P \int d^{2} q \int d \omega^{\prime} \operatorname{Im} \chi_{0}\left(\mathbf{q}, \omega^{\prime}\right) \\
& \times \frac{n_{\mathrm{B}}\left(\omega^{\prime}\right)+n_{\mathrm{F}}\left(\xi_{\mathbf{k}+\mathbf{q}}^{0}\right)}{\omega+\omega^{\prime}-\xi_{\mathbf{k}+\mathbf{q}}^{0}}
\end{aligned}
$$




$$
\begin{aligned}
\operatorname{Im} \Sigma(\mathbf{k}, \omega)= & -\frac{U^{2}}{8 \pi^{2}} \int d^{2} q \int d \omega^{\prime} \operatorname{Im} \chi_{0}\left(\mathbf{q}, \omega^{\prime}\right) \\
& \times\left[n_{\mathrm{B}}\left(\omega^{\prime}\right)+n_{\mathrm{F}}\left(\xi_{\mathbf{k}+\mathbf{q}}^{0}\right)\right] \delta\left(\omega+\omega^{\prime}-\xi_{\mathbf{k}+\mathbf{q}}^{0}\right),
\end{aligned}
$$

where $n_{\mathrm{F}, \mathrm{B}}$ denote Fermi and Bose distribution functions, respectively, $P$ is the principal value, and $\xi_{\mathbf{k}}^{0}=\varepsilon_{\mathbf{k}}-\mu$. These expression show that at frequencies lower than the temperature $|\omega| \ll \pi T$ (and, therefore, smaller than the first Matsubara frequency), the self-energy takes a clear nonFermi-liquid form for Fermi-surface vectors $\mathbf{k}_{\mathrm{F}}$ (see also Sec. VII C):

$$
\operatorname{Re} \Sigma\left(\mathbf{k}_{\mathrm{F}}, \omega\right) \stackrel{|\omega| \ll \pi T}{\sim}\left\{\begin{array}{cc}
-\omega \ln |\omega| & \mathbf{k}_{\mathrm{F}} \neq \mathbf{k}_{N} \\
\operatorname{sgn}(\omega) \sqrt{|\omega|} & \mathbf{k}_{\mathrm{F}}=\mathbf{k}_{N}
\end{array}\right.
$$

and

$\operatorname{Im} \Sigma\left(\mathbf{k}_{\mathrm{F}}, \omega\right)-\operatorname{Im} \Sigma\left(\mathbf{k}_{\mathrm{F}}, 0\right) \stackrel{|\omega| \ll \pi T}{\sim} \begin{cases}|\omega| & \mathbf{k}_{\mathrm{F}} \neq \mathbf{k}_{N} \\ \sqrt{|\omega|} & \mathbf{k}_{\mathrm{F}}=\mathbf{k}_{N},\end{cases}$

with $\mathbf{k}_{N}=(\pi / 2, \pi / 2)$.

The singular behavior of the self-energy at low frequency $|\omega| \ll \pi T$ comes from the singular behavior of the imaginary part of the susceptibility,

$$
\operatorname{Im} \chi_{0}(\mathbf{q}, \omega) \sim-\frac{\omega}{T} \ln \left|\frac{|\omega|-\omega_{s}(\mathbf{q})}{\Delta^{2}}\right|,
$$

where $\Delta \sim \pi T$ and

$$
\omega_{s}(\mathbf{q})=4\left|\sin \left(\left|q_{x}\right| / 2\right)-\sin \left(\left|q_{y}\right| / 2\right)\right| .
$$

The origin of these singularities, which appear at small frequency $\omega$ when $\mathbf{q}$ is near the diagonal, can be understood roughly from the fact that for any external momentum $\mathbf{q}$ located near the diagonal of the Brillouin zone, there are electrons near the half-filled diamond Fermi surface that can also be scattered to electrons near the Fermi surface.

More precisely, suppose that an electron below the Fermi surface at $\mathbf{k}-\mathbf{q} / 2$ is excited above the Fermi surface at $\mathbf{k}+\mathbf{q} / 2$ such that $\omega=\xi_{\mathbf{k}+\mathbf{q} / 2}^{0}-\xi_{\mathbf{k}-\mathbf{q} / 2}^{0}$. All internal momenta $\mathbf{k}$ in the bubble must be considered, while $\omega$ and $\mathbf{q}$ are fixed. But the velocity of the particle-hole excitation $\nabla_{\mathbf{k}}\left(\xi_{\mathbf{k}+\mathbf{q} / 2}^{0}-\xi_{\mathbf{k}-\mathbf{q} / 2}^{0}\right)$ vanishes at $\mathbf{k}=( \pm \pi / 2$, $\pm \pi / 2$ ) for any value of $\mathbf{q}$, which leads to van Hove-like singularities in the particle-hole excitations. The integral over $\mathbf{k}$ gives logarithmic contributions to $\operatorname{Im} \chi_{0}(\mathbf{q}, \omega)$ that are finite, except for $\omega=\omega_{s}(\mathbf{q})$, where they diverge when $\omega_{s}(\mathbf{q})$ is different from zero. Note that the singular frequency $\omega_{s}(\mathbf{q})$ vanishes when $\mathbf{q}$ is on the diagonal.
These singularities influence the low-frequency behavior of the imaginary part of the self-energy $\operatorname{Im} \Sigma\left(\mathbf{k}_{F}, \omega\right)$ as follows. We now consider the imaginary part in Eq. (C4) and integrate over the $\delta$ distribution to arrive at

$$
\begin{aligned}
\operatorname{Im} \Sigma(\mathbf{k}, \omega)= & -\frac{U^{2}}{8 \pi^{2}} \int d^{2} q \int d \omega^{\prime} \operatorname{Im} \chi_{0}\left(\mathbf{q}, \xi_{\mathbf{k}+\mathbf{q} / 2}^{0}-\omega\right) \\
& \times\left[n_{\mathrm{B}}\left(\xi_{\mathbf{k}+\mathbf{q} / 2}^{0}-\omega\right)+n_{\mathrm{F}}\left(\xi_{\mathbf{k}+\mathbf{q} / 2}^{0}\right)\right] .
\end{aligned}
$$

For the sake of detecting non-Fermi-liquid behavior, we are interested in the regime where the inequality $|\omega| \ll \pi T$ is satisfied. In that regime, it is the classical limit of the Bose function that dominates, and singularities come only from integrals of the logarithms in Eq. (C7), whose arguments are then ||$\xi_{\mathbf{k}+\mathbf{q} / \mathbf{2}}^{0}-\omega\left|-\omega_{s}(\mathbf{q})\right|$.

We take the integral over $q_{x}$ to run over the Brillouin zone; then, the integral over $q_{y}$ is restricted by the Bose function to a range near the diagonals where the inequality $\left|\xi_{\mathbf{k}+\mathbf{q} / 2}^{0}\right| \ll \pi T$ is satisfied. This process determines the limits of integration over $q_{y}$ to be of order $\delta q_{y} \sim \pi T / v_{F}$ away from the diagonal. The argument of the logarithm ||$\xi_{\mathbf{k}+\mathbf{q} / 2}^{0}-\omega\left|-\omega_{s}(\mathbf{q})\right|$, when expanded near the diagonal, can be approximated by

$$
\begin{gathered}
-\omega+\left(2 \sin \left(k_{y}-q_{x}\right) \pm 2 \cos \left(q_{x} / 2\right)\right) \delta q_{y} \\
\equiv-\omega+\alpha\left(k_{y}, q_{x}\right) \delta q_{y} .
\end{gathered}
$$

The integral over $q_{y}$ can be performed, leading to other logarithms that depend on $\alpha\left(k_{y}, q_{x}\right)$. The singular part of the remaining $q_{x}$ integral comes from regions where $\alpha\left(k_{y}, q_{x}\right)$ is small. The final result is obtained by expanding $\alpha\left(k_{y}, q_{x}\right)$ around values of $q_{x}$ where $\alpha\left(k_{y}, q_{x}\right)$ vanishes. In the Taylor expansion of $\alpha\left(k_{y}, q_{x}\right)$, there are terms linear in $\delta q_{x}$ that give the final $-|\omega|$ result for $\operatorname{Im} \Sigma\left(\mathbf{k}=\left(k_{x}, k_{y}\right), \omega\right)$ everywhere, except when the linear term in the expansion of $\alpha\left(k_{y}, q_{x}\right)$ vanishes, which occurs for $\mathbf{k}_{F}=( \pm \pi / 2$, $\pm \pi / 2)$. There, the leading term scales as $\delta q_{x}^{2}$, which modifies the integral over $q_{x}$ and leads to $\operatorname{Im} \Sigma\left(k_{x}, k_{y}, \omega\right)$ proportional to $-\sqrt{|\omega|}$. The Fermi points $\mathbf{k}_{F}=( \pm \pi / 2$, $\pm \pi / 2)$ seem special because it is there that the energy $\xi_{\mathbf{k}+\mathbf{q} / 2}^{0}-\xi_{\mathbf{k}-\mathbf{q} / 2}^{0}$ associated with particle-hole excitations can be small for the largest range of values of $\mathbf{q}$ near the diagonal.

\section{b. Numerical evaluation}

In Fig. 29, we show the numerical evaluation of these expressions for the real (first panel) and imaginary (second and third panel) parts for $U=2 t$ and $1 / T=16$, i.e., deep inside the metallic regime for the antinode (red), node (blue), and generic point $\mathbf{k}=(3 \pi / 4, \pi / 4)$ (light green). In both the real and imaginary parts, one can clearly see nonFermi-liquid behavior at low frequencies: Here, the slope of 

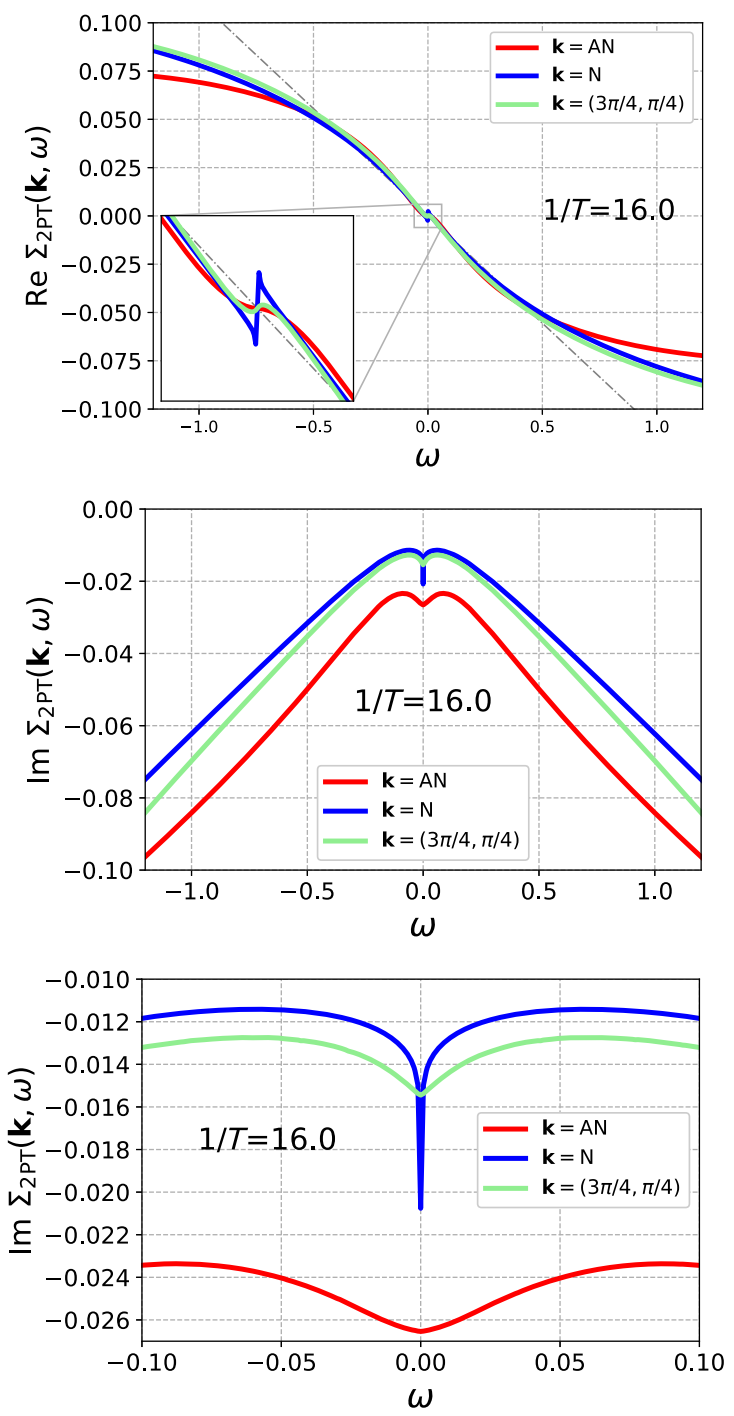

FIG. 29. Upper panels: real and imaginary parts of the selfenergy from $2 \mathrm{PT}$ at $1 / T=16$ for antinode (red), node (blue), and the generic point $\mathbf{k}=(3 \pi / 4, \pi / 4)$ (light green) on the realfrequency axis. The dotted-dashed line shows the respective result from a Fermi-liquid fit from the Matsubara axis.

the real part is reversed for the node and the generic $\mathbf{k}$-point while being nonlinear also for the antinode. For comparison, we also show (with the dotted-dashed line) the respective low-frequency result from the Fermi-liquid fit from the Matsubara axis (see later in this Appendix), which is oblivious to the features at $|\omega| \ll \pi T$.

Focusing on the imaginary part, one can observe that, despite their different magnitudes, the functional forms of the antinode and the generic point are very similar. However, the node exhibits a completely different functional behavior, eventually resulting in $|\operatorname{Im} \Sigma(\mathbf{k}=N, 0)|>$ $|\operatorname{Im} \Sigma(\mathbf{k}=(3 \pi / 4, \pi / 4), 0)|$ for very small frequencies, and it is indeed compatible with a square-root behavior. In addition to its frequency behavior, we extract the zerofrequency dependence on temperature, shown in the upper
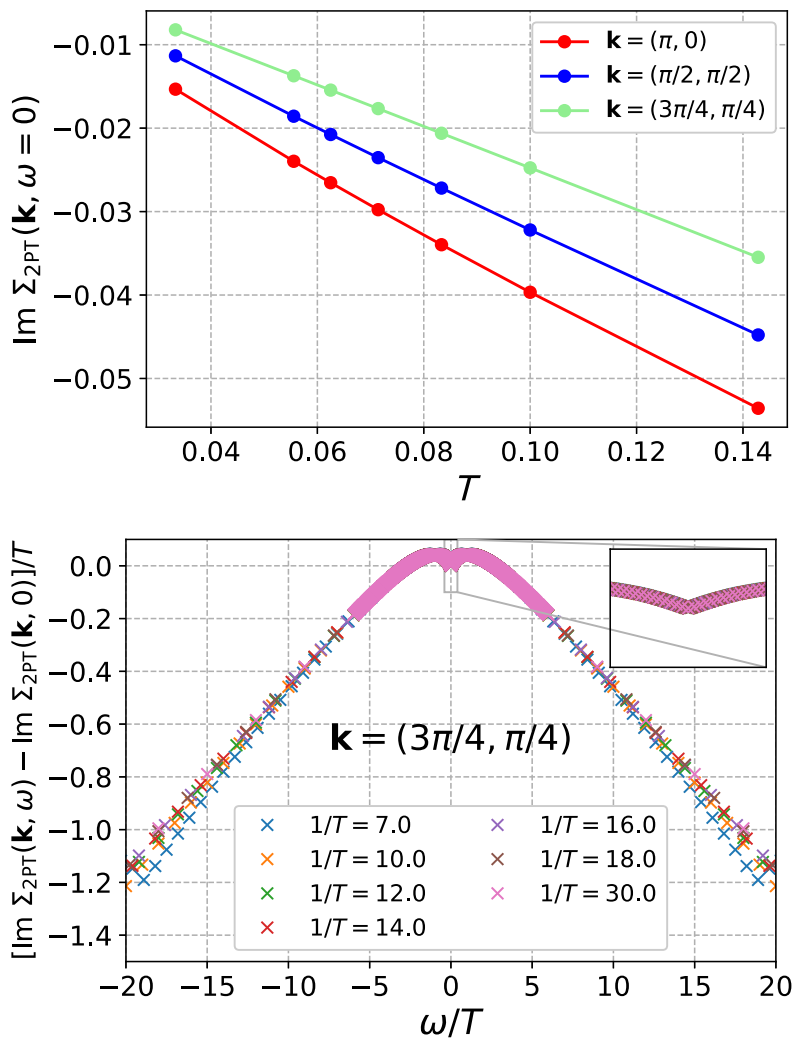

FIG. 30. Upper panel: imaginary part of the self-energy on the real axis at zero frequency and various Fermi-surface points calculated in second-order perturbation theory as a function of temperature. Lower panel: data collapse from $\omega / T$ scaling at the generic momentum point for several temperatures in the metallic regime. The inset shows a zoom to low frequencies.

panel of Fig. 30. Although log-corrections are expected [114], the dependence appears rather linear. Eventually, with our precise numerical data, we can numerically demonstrate that $\operatorname{Im} \Sigma(\mathbf{k}, \omega)$ indeed exhibits $\omega / T$ scaling as discussed in the main text, by plotting $(\operatorname{Im} \Sigma(\mathbf{k}, \omega)-\operatorname{Im} \Sigma(\mathbf{k}, 0)) / T$ over $\omega / T$ (lower panel of Fig. 30), which results in an excellent data collapse over a broad temperature (and frequency) range inside the metallic regime. However, as already stated in Appendix B, $\omega / T$ scaling typically applies in the quantum critical regime associated with QCPs controlled by a strongcoupling fixed point [167]. The QCP present is associated with the disappearance of antiferromagnetism at $U=0$, and hence, it is not clear whether $\omega / T$ scaling generally applies (e.g., logarithmic corrections may be expected).

\section{Imaginary frequencies and comparison}

In order to compare our findings on the real axis with the DiagMC benchmark, we directly evaluate Eq. (C1) on the Matsubara axis. We use meshes of $N_{i \omega}=200$ Matsubara frequencies and a linear momentum mesh of $N_{q}=N_{k}=$ 200 for the summations performed. Figure 31 shows the 2PT data (blue crosses) in comparison to the DiagMC benchmark (black circles and dashed line) for the antinode 

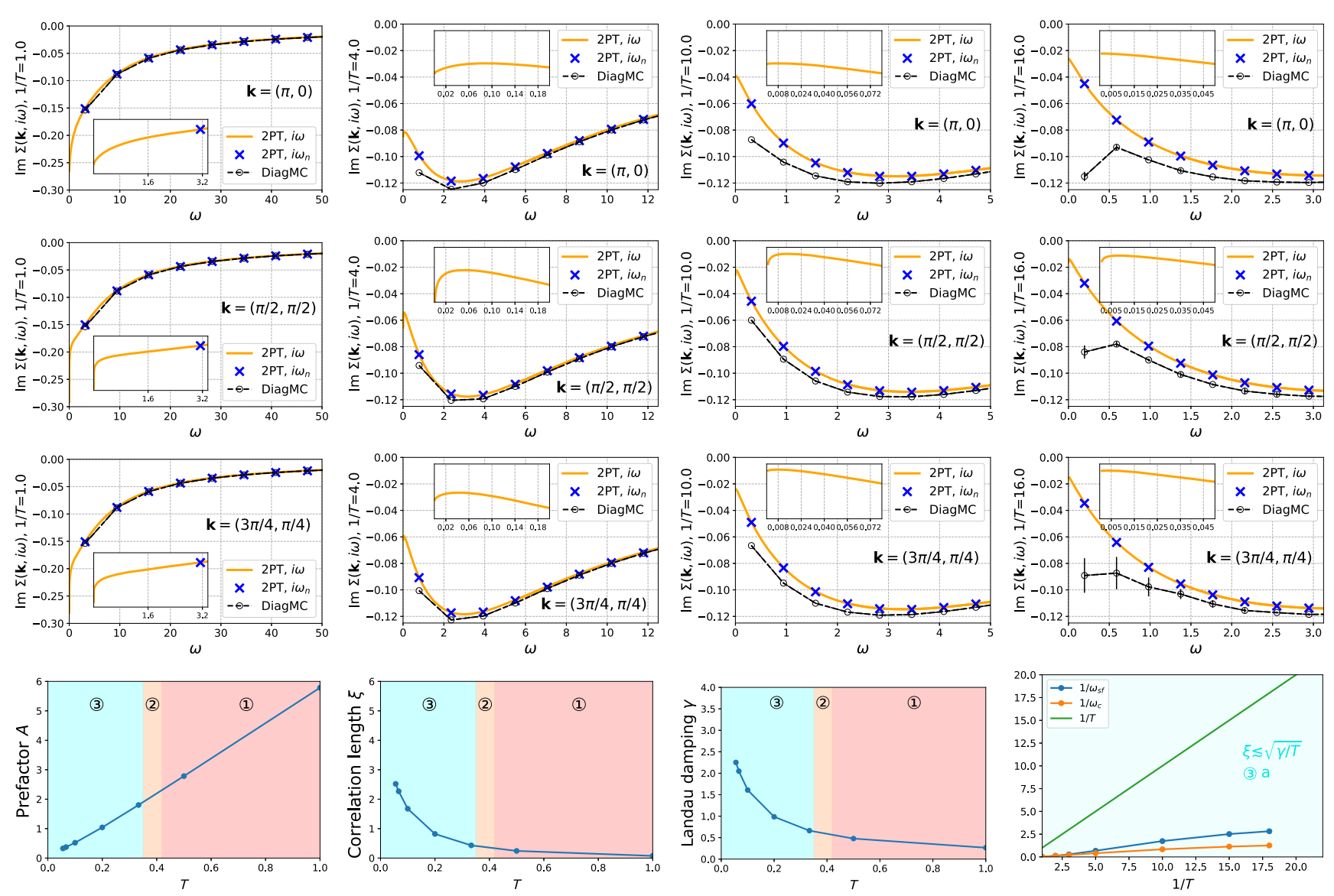

FIG. 31. Comparison of the imaginary part of the self-energy as a function of imaginary frequency for the antinode and node (first and second rows, respectively) obtained by 2PT (orange solid lines; blue crosses mark the Matsubara frequencies) to the DiagMC benchmark (black open circles, dashed lines) for various temperatures $(1 / T=1,4,10$, and 16 from left to right). The insets show a zoom of the lowest-frequency data (the interval range of the $y$ axis is constant for all $\mathbf{k}$-points shown in order to ensure comparability). Third row: results for $\mathbf{k}=(3 \pi / 4, \pi / 4)$. Lowest row: Ornstein-Zernike parameters (for the bubble) and energy scales for 2PT (obtained from a Matsubara fit) as functions of (inverse) temperature.

(uppermost row) and node (second row) as well as for the generic point $\mathbf{k}=(3 \pi / 4, \pi / 4)$ (third row) for various temperatures. The benchmark result is described quantitatively by $2 \mathrm{PT}$ in the incoherent regime and fairly well inside the high-temperature metallic regime. In particular, $2 \mathrm{PT}$ exhibits a clear momentum differentiation between the node and antinode. However, it fails in the description of the pseudogap; i.e., the regimes (4) and (5) are absent. This result is particularly apparent when comparing the emerging energy and length scales obtained from OrnsteinZernike fits (last row, cf. discussion in Sec. VIIC and Fig. 20).

However, as already discussed before, it may be quite difficult to judge this issue by looking at data from the Matsubara axis only. Therefore, we additionally compute the self-energy in 2PT on a continuous imaginary frequency grid (solid orange lines in Fig. 31; see insets for a zoom [168]). One indeed observes that, for frequencies much smaller than the ones accessible by a Matsubara grid $|\omega| \ll \pi T$, the slope of the imaginary part of the self-energy changes, at least in this fully nested case, to positive, yielding clear non-Fermi-liquid behavior. This behavior is particularly emphasized at the nodal point (second row).

Using a Fermi-liquid ansatz (see Appendix B), one can extract a momentum-dependent quasiparticle weight $Z_{\mathbf{k}}$ and inverse quasiparticle lifetime $\gamma_{\mathbf{k}}$ from the Matsubara axis (Fig. 32). As already discussed in the main text, these parameters are only meaningful (in the strict sense of describing Landau quasiparticles) if the low-frequency behavior of the self-energy is the "correct" one, i.e., compatible with the one expected by a Fermi liquid (e.g., in exhibiting a negative slope and a long quasiparticle lifetime). In order to estimate the quality of this plain Matsubara fit, we show the "offset" $-\operatorname{Im} \Sigma(\mathbf{k}, \omega \rightarrow 0)$ from both the real-frequency data (crosses in the lowest panel of Fig. 32) and the Matsubara fits. We notice that both methods of extraction are in very good agreement inside the metallic regime, except at the nodal point, where a severe underestimation by the Matsubara data of about $50 \%$ is found. 

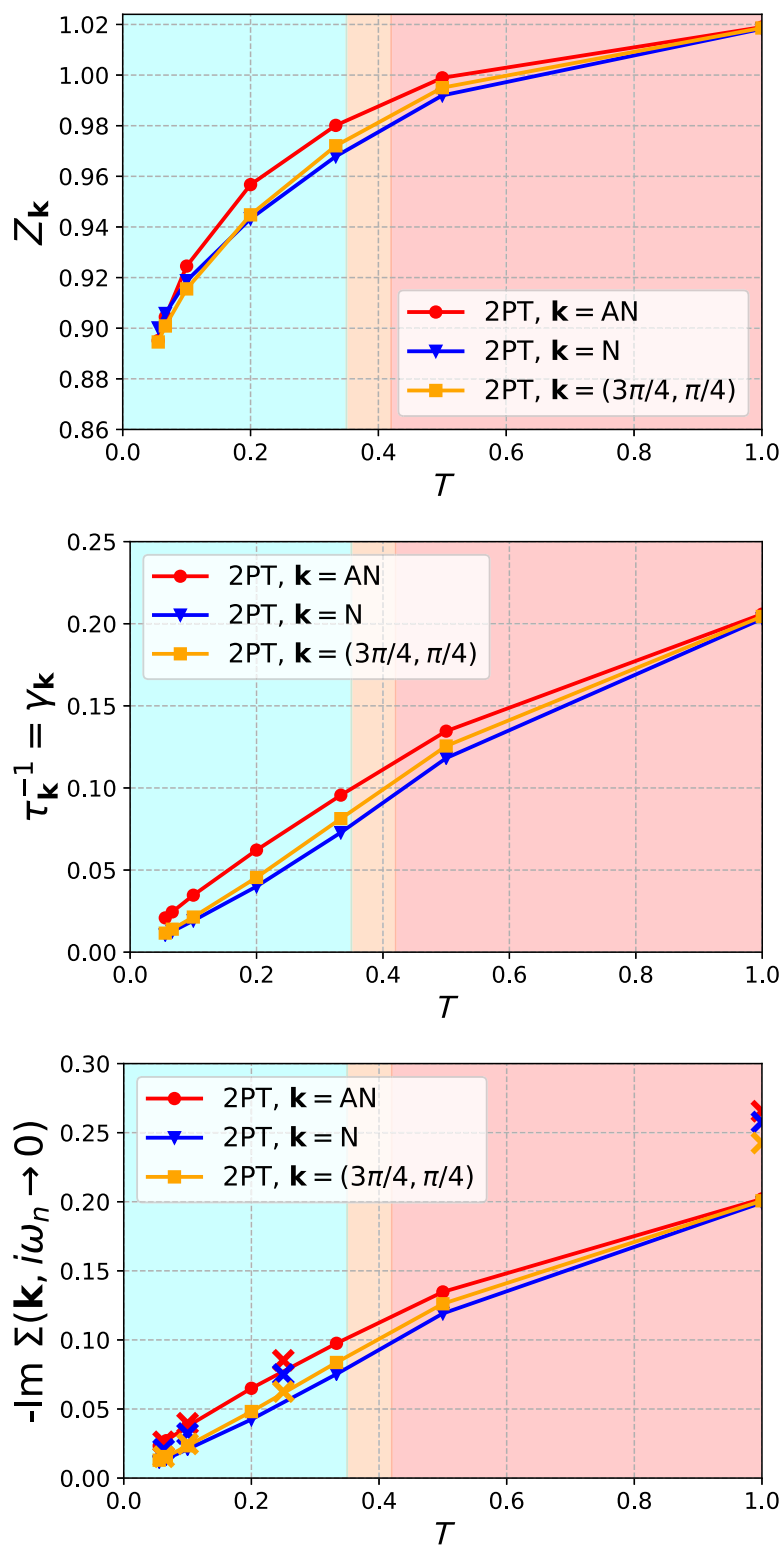

FIG. 32. Quasiparticle parameters $Z_{\mathbf{k}}$ (upper panel) and $\gamma_{\mathbf{k}}$ (middle panel) and the imaginary part of the self-energy extrapolated to zero frequency (lower panel) at the antinode (red circles), node (blue triangles), and $\mathbf{k}=(3 \pi / 4, \pi / 4)$ (orange squares) in second-order perturbation theory as a function of temperature. The values for the crosses in the lowest panel have been obtained directly from the real-frequency axis.

\section{APPENDIX D: BRIEF DESCRIPTION AND REFERENCES OF THE PRESENTED METHODS}

As one of the goals of this paper is a synopsis of a variety of different modern many-body techniques, in this Appendix, we give a short overview. Of course, the Appendix cannot serve as an exhaustive review, but it contains the basic idea of each method as well as the necessary references for further information and, if necessary, computational details for obtaining the results presented in this paper.

\section{DiagMC}

Diagrammatic Monte Carlo (DiagMC), first developed by Svistunov and Prokofiev [24], is based on the idea of stochastically sampling Feynman diagrams contributing to a perturbative expansion directly in the thermodynamic limit. In a broad sense, the diagrammatic Monte Carlo approach consists of the choice of a diagrammatic expansion for a given observable, a numerical algorithm computing the corresponding expansion coefficients, and, if necessary, the resummation techniques applied to the resulting perturbative series.

The physically most natural [169], skeleton expansions are potentially dangerous when applied to the two-dimensional fermionic Hubbard model, as they can converge to the wrong results in the vicinity of half-filling or whenever the local magnetic moment develops [123,126-128,136]. In contrast, bare expansions have a well-defined analytic structure and generally have a nonzero convergence radius in the twodimensional fermionic Hubbard model. Such expansions can be constructed around an arbitrary starting point $[160,170-173]$. The default choice $[174,175]$ is to expand around the noninteracting Hamiltonian with the chemical potential shifted by a constant ( $\alpha=U / 2$ at half-filling) [176], which effectively eliminates all diagrams with Hartree-type insertions, leading to a much improved variance, which is what we adopt here.

The choice of algorithm used to compute the expansion coefficients determines how large expansion orders can be obtained with reasonable error bars. In this paper, we employ the currently most efficient class of algorithms, based on the connected determinants diagrammatic Monte Carlo method (CDet) introduced for connected quantities by Rossi [25] and generalized to the evaluation of one-particle irreducible selfenergy diagrams in Refs. [27,48,177]. The particular selfenergy implementation used in this paper is $\Sigma D D M C$ from Ref. [26] as it is very well suited for the computation of specific k-points directly in momentum space. In its original formulation, CDet only works with bare diagrammatic expansions [consisting exclusively of interaction vertices and bare propagators $\left.G_{0}(\mathrm{r}, \tau)\right]$, although a generalization to semibold [170] and bold [122,178] schemes has recently been developed $[172,173]$. Contrary to previously developed diagrammatic Monte Carlo algorithms [121,122,174,175, 178-180] that evaluate a single Feynman diagram at each Monte Carlo step, CDet computes a factorial number of relevant diagrams permuted over all internal vertices at exponential cost, scaling with the expansion order $m$ as $o\left(3^{m}\right)$ for connected quantities [25] and $o\left(m^{2} 3^{m}\right)$ for oneparticle irreducible quantities [27,48,177] [or, alternatively, scaling as $o\left(m^{2} 2^{m}\right)$ and $o\left(m^{4} 2^{m}\right)$, respectively [181] ]. This improvement is achieved by grouping all possible diagram topologies of a particular diagram order into determinants and subsequently subtracting all disconnected, and in the case of the self-energy, also all one-particle-reducible, diagrams from the sum by using a recursive formula. As a consequence of the exponential scaling, the total error of a convergent series scales polynomially with computational 
time [182]. The statistical variance of the algorithm only allows for the computation of a finite number of expansion coefficients, typically $m \sim 10-12$ (compared to $m \sim 6-7$ for previous diagrammatic Monte Carlo algorithms).

In challenging cases, such as low temperatures and/or high values of physical coupling, resummation techniques are needed to evaluate the series close to, or beyond, the radius of convergence, which constitutes the only source of systematic error in this approach. A protocol for the resummation of extrapolation, which gives control over this systematic error, has been developed in Ref. [26].

At half-filling, every other order is equal to zero for the double occupancy (even) as well as for the self-energy evaluated at every k-point on the noninteracting Fermi surface (odd). A consequence of particle-hole symmetry at half-filling is that a single calculation of the expansion coefficients at a given temperature yields results for all values of $U$, provided the series is resummable (away from half-filling, this is still the case; however, the evaluated density $n$ also changes as a function of interaction strength $U$ ). For all presented observables, the limiting factor to the applicability of this approach at high enough interactions and low enough temperatures is the presence of singularities in the vicinity of the positive real axis in the complex plane of interaction strength $U$, which render the resummation of the series prohibitively difficult. Despite the fact that, in this work, series have only been evaluated within their radius of convergence, resummation by means of Padé and Dlog-Padé approximants [26,183,184] has been performed in order to accelerate the convergence of series while controlling the systematic error of the extrapolations. In the half-filled two-dimensional Hubbard model, this approach has previously allowed for numerically exact results that shed light on the metallic to quasi-antiferromagnetic crossover as described by the self-energy [48] as well as various other thermodynamic observables $[49,185]$.

\section{DQMC}

The determinantal quantum Monte Carlo (DQMC) algorithm, formulated by Blankenbecler, Scalapino, and Sugar [23], is a controlled method and has been widely applied to finite-temperature simulations of correlated fermion systems. Its basic methodology is to transform two-body interactions into free fermions coupled with auxiliary fields and then sample the fields to compute fermionic observables. To achieve this goal, a Trotter decomposition $\left[e^{-\Delta \tau \hat{H}}=e^{-\Delta \tau \hat{H}_{0} / 2} e^{-\Delta \tau \hat{H}_{I}} e^{-\Delta \tau \hat{H}_{0} / 2}+\right.$ $\mathcal{O}\left[(\Delta \tau)^{3}\right]$ is used in this work, where $\hat{H}_{0}$ and $\hat{H}_{1}$ are the tight-binding and interacting parts of the model Hamiltonian] and a Hubbard-Stratonovich (HS) transformation (the discrete spin decomposition [186] for an on-site Hubbard interaction is used in this work) are applied within the discretization for the inverse temperature as $\beta=M \Delta \tau$. When convergence $\Delta \tau \rightarrow 0$ can be established, the method is numerically exact.
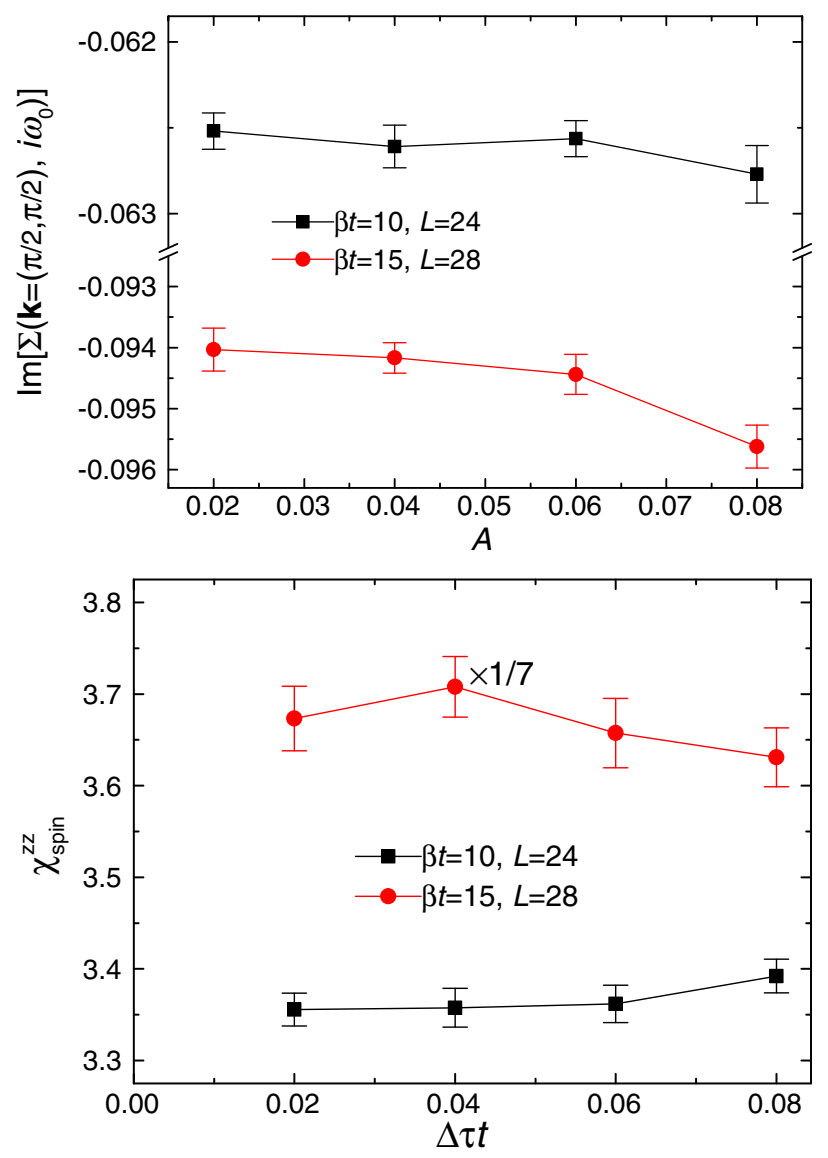

FIG. 33. DQMC: Trotter extrapolation for the lowest Matsubara frequency of the imaginary part of the self-energy at the node (upper panel) and the magnetic susceptibility at the antiferromagnetic wave vector (lower panel).

The systematic error from finite $\Delta \tau$ is controllable and can be eliminated by extrapolating simulations with several different $\Delta \tau$ values. In Fig. 33, we present representative results of systematic Trotter errors in DQMC simulations. Two systems, $L=24$ with $\beta t=10$ and $L=28$ with $\beta t=15$, are studied. We concentrate on the self-energy at $\mathbf{k}=(\pi / 2, \pi / 2)$ (upper panel) and the spin susceptibility (lower panel). The results for both parameters show convergence to the $\Delta \tau=0$ limit for $\Delta \tau t \leq 0.06$ within our statistical uncertainty, which means that our choice of $\Delta \tau t \leq 0.02$ is reliable.

Further details about the DQMC algorithm can be found in several review papers $[187,188]$. We have also implemented our most recent improvements [189,190] of this method in this work. The minus-sign problem is absent for the Hubbard model at half-filling studied in this work, and the DQMC method can reach large system sizes. For the calculation of dynamical quantities, the imaginary-time single-particle Green function and correlation functions are measured in the DQMC simulation, and then, the imaginary-frequency observables are obtained through the Fourier transformation. The Dyson equation is then applied 
to compute the self-energy. For the $U / t=2$ case, convergence to the thermodynamic limit of all the physical observables is observed for up to $L=32$ for $\beta t \leq 10$. For lower temperatures, we perform finite-size scalings using second-order polynomials with constraints of monotonic behaviors to reach the thermodynamic limit. We typically use between $10^{4}$ and $3 \times 10^{5}$ measurement samples for systems with linear system size from $L=20$ to $L=48$. In $\mathrm{DQMC}$, the number of Matsubara frequencies is determined by the number of imaginary-time slices $M$, which in turn depends on the Trotter step, chosen here to be $\Delta \tau=$ 0.02 as mentioned earlier.

\section{MFT}

The calculation of the static mean-field theory (MFT) susceptibility is analytically equivalent to the (non-selfconsistent) random phase approximation (RPA). The idea of the RPA, originally introduced in Ref. [191], is building ladders for the susceptibilities with the bare interaction $U$ in the physically relevant channel, utilizing the noninteracting susceptibility Eq. (C2). Thus, the fermionic propagator is the noninteracting Green function $G_{0}\left(\mathbf{k}, i \omega_{n}\right)$. The interacting susceptibility is obtained via

$$
\chi_{\mathrm{sp}}^{\mathrm{RPA}}\left(\mathbf{q}, i \Omega_{n}\right)=\frac{2 \chi_{0}\left(\mathbf{q}, i \Omega_{n}\right)}{1-U \chi_{0}\left(\mathbf{q}, i \Omega_{n}\right)} .
$$

We use a momentum grid with maximum linear mesh sizes of $N_{q}=128$ and $N_{k}=128$ and the number of fermionic Matsubara frequencies being $N_{i \omega}=1000$.

\section{DMFT}

The dynamical mean field theory (DMFT) has become one of the standard techniques for tackling strongly correlated systems over the past decades. Its basic idea consists in mapping the full lattice Hamiltonian Eq. (1) onto a self-consistently determined single-site Anderson impurity model. This procedure is exact in infinite dimensions, but it represents an approximation in finite dimensions because it neglects spatial correlations. Hence, the selfenergy in DMFT is purely local. For further details of the algorithm, we recommend the seminal papers $[9,10]$ and the review [28]. The self-energy and magnetization data presented in this paper have been produced using a state-ofthe-art continuous-time quantum Monte Carlo impurity solver in its interaction expansion (CT-INT $[176,192,193]$ ), which is, like the DMFT self-consistency scheme, entirely implemented in the TRIQS framework [194]. For the calculation of the (magnetic) susceptibility and correlation length, we used data from both (i) an exact diagonalization (with four bath sites) impurity solver and the implementation of the Bethe-Salpeter equations presented in Ref. [195] and (ii) the CT-INT solver and two-particle response function framework of TRIQS [196], carefully cross- checking that they obtain the same results. As the magnetic correlations (and correlation lengths) grow exponentially, reaching low temperatures, we used a very dense momentum grid for these data, with maximum linear mesh sizes of $N_{q}=200$ and $N_{k}=200$ and the number of fermionic Matsubara frequencies being $N_{i \omega}=160$. In the case of the CT-INT impurity solver, we used $N_{\text {cycles }}=1.2 \times 10^{7}$ Monte Carlo steps.

\section{Cluster extensions of DMFT a. DCA}

The dynamical cluster approximation (DCA) $[29,67,68]$ is an embedding technique wherein the electron self-energy is obtained from the solution of an impurity model with $N_{c}$ interacting sites coupled to an infinite bath. As such, the DCA is just one particular generalization to $N_{c}>1$ of the "single-site" dynamical mean-field method that becomes exact as the number of impurity sites $N_{c} \rightarrow \infty[10,28,29]$. The DCA formulation partitions the Brillouin zone into $N_{c}$ equal-area tiles and approximates the self-energy in each tile $a, \Sigma_{a}$ as a piecewise constant function of momentum as

$$
\Sigma(\mathbf{k}, \omega)=\sum_{a=1 \ldots N_{c}} \phi_{a}(\mathbf{k}) \Sigma_{a}(\omega)
$$

where $\phi_{a}(\mathbf{k})=1$ if $\mathbf{k} \in a$ and $\phi_{a}(\mathbf{k})=0$ for $\mathbf{k} \notin a$. Similar to DMFT, the DCA invokes a self-consistency loop where an initial guess for the impurity model parameters produces a set of $\Sigma_{a}$, which are then used to update the bath. In this work, we obtain results for large values of $N_{c}$ in the paramagnetic phase but note that, at the weak-coupling and low temperatures studied in this work, the DCA expansion in cluster size $N_{c}$ does not appear to be in a scaling regime, and therefore, one needs much larger clusters in order to estimate the infinite-system-size limit $[48,197]$.

To solve the $N_{c}$-site impurity problem, we use the continuous-time auxiliary-field method [193,198] with submatrix updates [199]. Similar to other methods that employ Hubbard-Stratonovich decoupling, the primary computational hurdle is finding determinants of an $m \times m$ matrix, where $m$ is referred to as the expansion order. Unlike Hirsch-Fye solvers that employ a fixed $\Delta \tau$, the expansion order and time steps in the continuous-time auxiliary-field method (CT-AUX) are not fixed - an important improvement that removes the necessity for a $\Delta \tau \rightarrow 0$ extrapolation. In the case of the DCA, applying CT-AUX to clusters of size $N_{c}$ results in an expansion order $m \propto \beta U N_{c}$ [73]. In this work, we have presented data primarily for fixed cluster size $N_{c}=128$ on a bipartite cluster. The DCA self-consistency is presumed to be paramagnetic and is iterated approximately 15-20 times until the deviation between iterations is much less than the statistical error obtained from approximately $2 \times 10^{6}$ samples of each frequency up to $n=1024$. Our DCA and CT-AUX codes are based on the ALPS libraries [200,201]. 


\section{b. CDMFT}

Cellular dynamical mean-field theory (CDMFT) is the real-space cluster extension of DMFT [29,77,81,202]. While proposed around the same time, it has so far been less widely used than the complementary dynamical cluster approximation, which is formulated in momentum space. The impurity of the auxiliary Anderson model in CDMFT, contrary to single-site DMFT, usually consists of superstructures obtained by upfolding the lattice unit cell. On the square lattice, e.g., these superstructures may consist of $N \times N$ quadratic patches. Hence, nonlocal correlations are included on length scales given by the cluster size as intersite single-particle self-energies. Even within the aforementioned patch geometries, which typically retain pointgroup symmetry, translational symmetry is broken (for $N>2$ even within the cluster). Reperiodization schemes suffer from ambiguity and may even lead to convergence problems when attempted inside the self-consistency loop. The lattice quantities are therefore approximated from the converged CDMFT solution by restoring the translational invariance for the Green function, the self-energy, or its cumulants $[32,70,202-207]$. This study uses the cumulant scheme presented in Appendix B of Ref. [71].

A recent study [71] has shown that a so-called centerfocused extrapolation of self-energies (and even susceptibilities) to infinite cluster sizes (with a linear regression in $1 / N$ ) yields the best results in comparison to numerically exact diagrammatic quantum Monte Carlo calculations. The CDMFT + CFE self-energies presented in this paper have been obtained by considering quadratic patches of up to $N=8$ for the extrapolation scheme. The auxiliary impurity models were solved using a state-of-the-art continuous-time quantum Monte Carlo impurity solver in its interaction expansion (CT-INT [176,192,193]), implemented in the TRIQS framework [194]. We used 20 CDMFT self-consistency loops (until convergence). Afterwards, we performed 30 simulations with different random seeds starting from this previously converged solution. We take the self-energy as the mean of these 30 simulations. The simulations start with 10000 warmup cycles, followed by $4 \times 10^{6}$ measurement cycles (the length of a cycle is 300 Monte Carlo steps).

For the calculation of the magnetic susceptibility, we applied an antiferromagnetic staggered field $H$ and measured the staggered magnetization $m$. We then linearized the $m-H$ curve at small fields:

$$
\operatorname{Re} \chi\left(\mathbf{q}=(\pi, \pi), i \Omega_{n}=0\right)=\left.\frac{\partial m}{\partial H}\right|_{H=0} .
$$

Figure 34 shows a sample of these curves for various temperatures. The transition to an antiferromagnetic ordered state is signaled as a vertical tangent at $H=0$, i.e., as a divergence of the susceptibility, at $1 / T_{\text {Néel }} \approx 13.7$. This temperature represents a very small correction with respect to DMFT, where $1 / T_{\text {Néel }} \approx 12.5$.

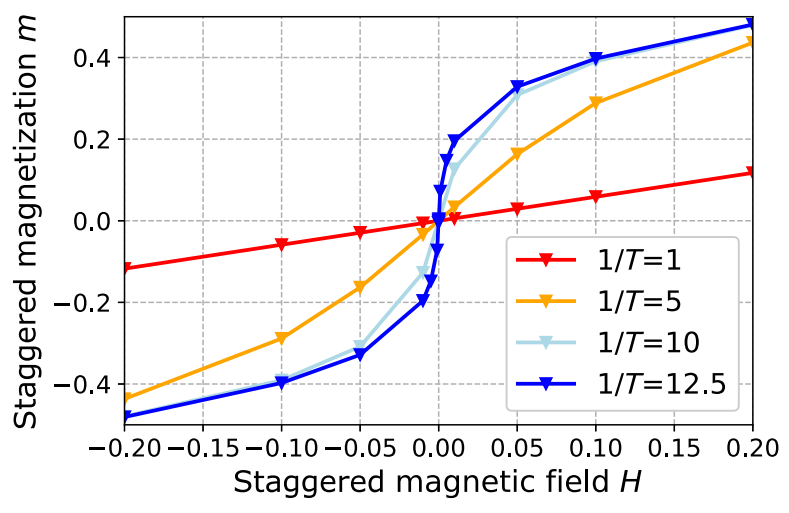

FIG. 34. Antiferromagnetic staggered magnetization $m$ vs staggered applied field $H$ in CDMFT on an $8 \times 8$ lattice for various temperatures.

\section{Diagrammatic extensions of DMFT}

A different route for the inclusion of spatial correlations on top of DMFT is to take its diagrammatic extensions. The principle is to extract a higher-order correlation function from an auxiliary impurity model and consistently calculate the desired observables from it. For a very recent overview of diagrammatic extension of DMFT, see Ref. [33]. More detailed information for each method is given below.

\section{a. DГA}

In the dynamical vertex approximation (DГA) [53,106], the two-particle analog of the self-energy, the fully irreducible two-fermion scattering vertex $\Lambda$, in the spirit of DMFT, is assumed to be purely local. In order to obtain the corresponding susceptibilities and self-energies, without the knowledge of leading instabilities, the parquet equations have to be solved self-consistently (see Refs. [208,209] for nanoscopics, Ref. [210] for $\pi$-tons, and Refs. [62,211] for the two-dimensional Hubbard model). However, if (like in the present case of the Hubbard model) the leading instability is known, the scheme can be significantly facilitated by considering only the Bethe-Salpeter equations in the associated scattering channel and the Dyson-Schwinger equation, in order to obtain susceptibilities and self-energies (for successful applications, see Refs. [47,52,72,153,154,209,212-217]). In the vicinity of a phase transition, in order to restore the proper sum rules for the susceptibilities (and, thus, the asymptotics of the self-energy and, therefore, the twoparticle self-consistency), within the ladder version of the DГA, a Moriyaesque so-called $\lambda$ correction is used $[51,53,106,218]$. For the results presented in the main text of the current paper, this correction is only done for the spin susceptibility $\left(\lambda_{\mathrm{sp}}\right.$, see below). For the calculation of the (magnetic) susceptibility and correlation length, we used data from an exact diagonalization (with four bath sites) impurity solver, and the implementation of the BetheSalpeter equations and Dyson-Schwinger equation 
presented in Ref. [195] (again, like in the DMFT case) was cross-checked with CT-INT. We used a momentum grid with maximum linear mesh sizes of $N_{q}=200$ and $N_{k}=$ 200 and the total number of fermionic, as well as bosonic Matsubara frequencies, being $N_{i \omega}=N_{i \Omega}=160$. For completeness, it is instructive to version of a Moriyaesque $\lambda$ correction [51,53], it is instructive to also compare the results obtained with the correction in both particle-hole channels (i.e., charge and spin, $\lambda_{\text {sp }}+\lambda_{\text {ch }}$; see Eq. (6) in Ref. [51])

$$
\frac{1}{\beta} \sum_{\Omega, \mathbf{q}} \chi_{r, \mathbf{q}}^{\Omega, \lambda_{r}}=\frac{1}{\beta} \sum_{\Omega} \chi_{r}^{\Omega}
$$

[with $r=(\mathrm{ch}, \mathrm{sp})]$ to the ones obtained with only a correction in the spin channel $\left[\lambda_{\mathrm{sp}}\right.$, Eq (5) in Ref. [51]]:

$\frac{1}{\beta} \sum_{\Omega, \mathbf{q}} \frac{1}{2}\left[\chi_{\mathrm{sp}, \mathbf{q}}^{\Omega, \lambda_{\mathrm{sp}}}+\chi_{\mathrm{ch,} \mathbf{q}}^{\Omega}\right]=\frac{1}{\beta} \sum_{\Omega} \chi_{\uparrow \uparrow}^{\Omega, \mathrm{AIM}}=\frac{n}{2}\left(1-\frac{n}{2}\right)$.

Results for both schemes are presented in Fig. 35. In comparison with DiagMC in Fig. 10, the self-energy obtained with $\lambda_{\mathrm{sp}}+\lambda_{\mathrm{ch}}$ would lead to a slightly better agreement of the imaginary part at low frequencies at the antinode in comparison to the benchmark (left and central panels) but a slightly worse agreement for the susceptibility, the correlation length, and $T_{*}$ (right panel). It should be noted that the spin susceptibility and correlation length of DГA with $\lambda_{\mathrm{sp}}$ agree almost perfectly with a version of TPSC + , coined TPSC $+\mathrm{GG}$, down to low $T$. A more detailed comparison of different TPSC variants is given in Appendix D 11. Eventually, in order to be able to compare the simple DГAesque approximation discussed in Sec. VII $\mathrm{D}$, we recall the Dyson-Schwinger equation of motion for the ladder version of the DГA (see Refs. [53,106,219], here omitting the Hartree term):

$$
\begin{aligned}
\Sigma\left(\mathbf{k}, i \omega_{n}\right)= & \frac{U T^{2}}{2} \sum_{\mathbf{q}, i \Omega_{n}}\left[\gamma_{\mathrm{ch}, \mathbf{q}}^{\omega \Omega}-3 \gamma_{\mathrm{sp}, \mathbf{q}}^{\omega \Omega}+2\right. \\
& +U \gamma_{\mathrm{ch}, \mathbf{q}}^{\omega \Omega} \chi_{\mathrm{ch}, \mathbf{q}}^{\Omega}+3 U \gamma_{\mathrm{sp}, \mathbf{q}}^{\omega \Omega} \chi_{\mathrm{sp}, \mathbf{q}}^{\Omega} \\
& -\sum_{\mathbf{k}^{\prime}, i \omega_{n}^{\prime}}\left(F_{\mathrm{ch}}^{\omega \omega^{\prime} \Omega}-F_{\mathrm{sp}}^{\omega \omega^{\prime} \Omega}\right) \\
& \left.\times G\left(\mathbf{k}^{\prime}, i \omega_{n}^{\prime}\right) G\left(\mathbf{k}^{\prime}+\mathbf{q}, i \omega_{n}^{\prime}+i \Omega_{n}\right)\right] \\
& \times G\left(\mathbf{k}+\mathbf{q}, i \omega_{n}+i \Omega_{n}\right),
\end{aligned}
$$

where $\chi$ refers to the physical, Moriya-corrected susceptibility, $F$ denotes the full two-particle vertex from the self-consistently determined Anderson impurity model in DMFT and $G:=G_{\mathrm{DMFT}}$, and the last line of the equation accounts for double-counting corrections of the local part of the self-energy. Here, $\gamma$ denotes the electron-boson coupling vertex (already integrated over the internal momentum k). For the simple approximation in Sec. VII D, the electron-boson coupling vertex is set to unity for all momenta and frequencies. A precise definition of the components of the equation of motion can be found in Eq. (4.19) of Ref. [219].

\section{b. TRILEX}

The triply irreducible local expansion (TRILEX) method is a relatively recent approach to strong correlations, which utilizes the decoupling of the fermion-fermion interaction of the Hubbard Hamiltonian into auxiliary bosons
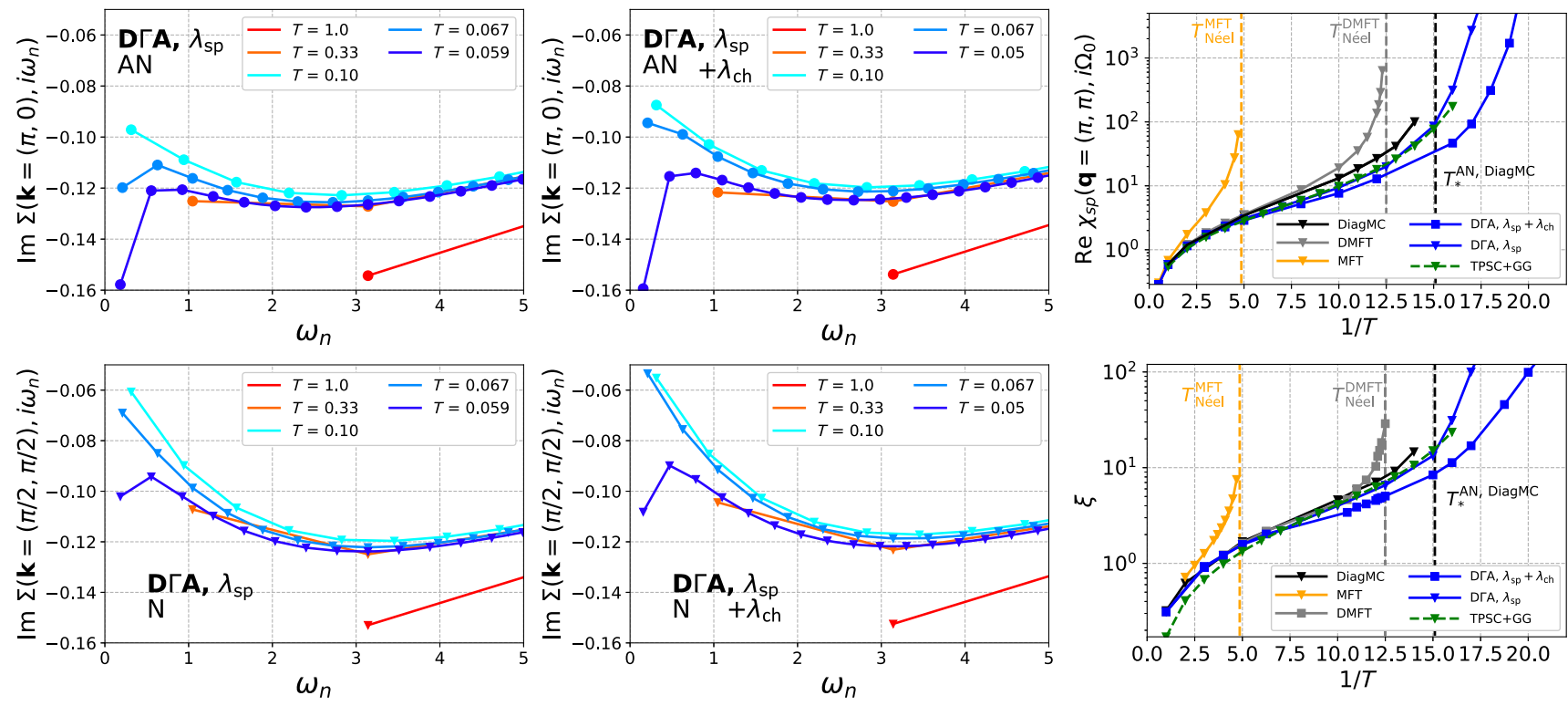

FIG. 35. Results from alternative Moriya correction schemes in ladder DГA for various quantities. Note that the lowest temperatures shown for the self-energy do not agree, in order to show the full gap in both schemes. 
$[220,221]$. For these bosons, the fermion-boson vertex of a self-consistently determined impurity model is extracted, and the lattice polarization and self-energy are calculated via Hedin's equations. The beauty of this approach consists in the facts that (i) the fermion-boson vertex is relatively easy to calculate (compared to the four-point vertex), (ii) the convergence of the method to the exact solution can be achieved via a cluster extension of TRILEX [222] (we used DCA clusters with two and four sites in this paper), and (iii) the extension for treating superconductivity is straightforward [223]. However, the interaction is not unique, and it comes at the price of introducing an additional (Fierz) parameter $\alpha$, which, in this paper, is set in such a way that we decouple only the spin channel (i.e., $\alpha=1 / 3$ in Heisenberg spin decoupling [221]). This choice can be motivated by a so-called fluctuation diagnostics analysis of the self-energy in the pseudogap regime of the Hubbard model [88]. A different route to enhance the results of TRILEX can be made by inserting the fermion-boson vertex on both sites of the Hedin equations (an approach that we call TRILEX $\Lambda^{2}$; see also Refs. [224,225] for a similar idea in the dual theories; see also Ref. [226] for an efficient evaluation of the polarization bubble in DMFT and Ref. [227] for the question of conservation in two-particle self-consistent theories). The self-energies in the main text using this method show results that turn out to be similar to $N_{c}=4$ cluster TRILEX. We used momentum grids with maximum linear mesh sizes of $N_{q}=128$ and $N_{k}=128$, and the number of fermionic and bosonic Matsubara frequencies were chosen as $N_{i \omega}=200$ and $N_{i \Omega}=20$, respectively. For the impurity solver (CT-INT [176,192,193]), we used $N_{\text {cycles }}=6.9 \times 10^{7}$ Monte Carlo steps.

\section{c. DF}

The dual-fermion (DF) approach [228-230] is a diagrammatic extension of the single-site DMFT, motivated by the idea that nonlocal corrections to DMFT can be captured by a perturbative expansion around a solution of the dynamical mean-field impurity problem.

In the dual-fermion formalism [228,231], one replaces the lattice problem with a lattice of coupled Anderson impurity problems, resulting in an action of the form

$S\left[f, f^{*}\right]=\sum_{\omega k \sigma} \mathrm{g}_{\omega}^{-2}\left(\left(\Delta_{\omega}-\epsilon_{k}\right)^{-1}+\mathrm{g}_{\omega}\right) f_{\omega k \sigma}^{*} f_{\omega k \sigma}+\sum_{i} V_{i}$,

with $V_{i} \equiv V\left[f_{i}^{*}, f_{i}\right]$, and where $\mathrm{g}_{w}$ is the momentumindependent Green function of the Anderson impurity problem; $\Delta_{\omega}$ is the hybridization function between the impurity and the bath $[28,228]$. The dual-fermion action now depends on $f$ and $f^{*}$, which are dual operators obtained via a Hubbard-Stratonovich transformation, and in this dual space, the interaction terms become local and are collected in the function $V_{i}$ [232-234]. Correctly representing $V_{i}$ remains problematic due to the complexity of higher-leg vertex functions [235,236], so we truncate the vertex at the level of four-leg operators, although higherorder contributions may be important $[33,237,238]$. Once the dual self-energy $\tilde{\Sigma}(k, \omega)$ is obtained, the lattice selfenergy is given by

$$
\Sigma(k, \omega)=\frac{\tilde{\Sigma}(k, \omega)}{1+\mathrm{g}_{\omega} \tilde{\Sigma}(k, \omega)}+\Sigma^{\mathrm{DMFT}}(\omega) .
$$

We present DF results from the open-source opendf code [231], starting from a single-site $\left(N_{c}=1\right)$ dynamical meanfield solution obtained with a CT-AUX [73,193,199] impurity solver. The method treats all local correlations in a nonperturbative manner via DMFT and then perturbatively includes nonlocal correlations via a restricted set of self-consistent ladder diagrams for the nonlocal ("dual") self-energy in the charge and spin channels, which is known as the self-consistent ladder dual-fermion method [230]. From this method, one can also extract two-particle spin susceptibilities, which we present. However, there is no self-consistency on the two-particle level, and as such, the status of two-particle susceptibilities from DF is known to be approximate and expected to maintain only qualitative correctness [239]. In all cases, the input DMFT solutions (Green functions and vertices) are obtained using a CTAUX method [193].

DMFT results for the full vertex $F^{\nu, \nu^{\prime}, \omega}$ are obtained with a frequency truncation in $\nu, \nu^{\prime}, \omega$. We present results with truncations $\left|\nu_{c}\right|=\left|\nu_{c}^{\prime}\right|=96$ in fermionic frequencies and $\left|\omega_{c}\right|=64$ in bosonic frequencies. The DF result is not strongly dependent upon this truncation. More important is the known sensitivity of the result to the momentum resolution [240]. As such, we employ a $64 \times 64 \mathrm{k}$-space grid in the DF self-consistency. Further, the result for the lattice self-energy is extremely sensitive to the value of the dual self-energy due to the inversion in Eq. (C7). We iterate the DF self-consistency until convergence on the scale of $1 \times 10^{-8}$. We also refer to a recent study of the doped Hubbard model in a dual parquet scheme [241,242] and a dual parquet solution of the Falicov-Kimball model [243].

\section{d. DB}

The dual boson (DB) approach [244-246] is an extension of the DF approach that, additionally to the local Coulomb interaction, accounts for the effect of nonlocal interactions in different bosonic channels ( $\varsigma$ ). Within the DB approach, local electronic correlations are considered exactly in the framework of the (extended) dynamical mean-field theory (EDMFT) [247,248]. Nonlocal collective fluctuations are treated diagrammatically beyond the EDMFT level. For this aim, dual-boson fields $\varphi^{\varsigma}$ are introduced in addition to dual-fermion variables $f^{(*)}$ that are already present in the DF approach. The DB theory is 
derived analytically using a path-integral formalism, so many existing EDMFT-based approaches can be obtained as a certain approximation of the DB method [225,246]. Also, the DB theory fulfills the Mermin-Wagner theorem, which allows us to avoid unphysical phase transitions in two dimensions [249].

The action of the DB theory is as follows:

$$
\tilde{\mathcal{S}}=-\sum_{\mathbf{k}, \omega, \sigma} f_{\mathbf{k} \omega \sigma}^{*} \tilde{\mathcal{G}}_{\mathbf{k} \omega \sigma}^{-1} f_{\mathbf{k} \omega \sigma}-\frac{1}{2} \sum_{\mathbf{q}, \Omega, \zeta} \varphi_{\mathbf{q} \Omega}^{\varsigma} \tilde{\mathcal{W}}_{\mathbf{q} \Omega}^{s-1} \varphi_{-\mathbf{q},-\Omega}^{\varsigma}+\tilde{\mathcal{F}}
$$

Here, the bare fermion $\tilde{\mathcal{G}}_{\mathbf{k}, \nu, \sigma}$ and boson $\tilde{\mathcal{W}}_{\mathbf{q}, \omega}^{\varsigma}$ propagators are given by nonlocal parts of the EDMFT Green function and renormalized interaction [246], respectively.

The interaction part $\tilde{\mathcal{F}}\left[f^{*}, f, \varphi\right]$ of the dual action contains all possible, exact, local fermion-fermion and fermion-boson vertex functions of the impurity problem. Here, as well as in most of the DB approximations, we restrict ourselves to the lowest-order (two-particle) interaction terms that are given by the four-leg fermion-fermion and three-leg fermionboson vertex functions. This truncation of the interaction allows us to describe collective charge $[250,251]$ and spin $[252,253]$ degrees of freedom in a conserving way using the ladder DB approximation [244-246,254].

In the main part of the text, only single-shot ladder dualboson results are discussed. These calculations are performed on the basis of the converged DMFT solution of the problem, where the bosonic hybridization function is equal to zero. Importantly, in the latter case, the DB theory fully coincides with the DF approach if only the local Coulomb interaction is considered. The corresponding local impurity problem is solved using the open-source CT-HYB solver [255,256] based on the ALPS libraries [257]. This solution requires $N_{\text {cycles }}=8.1 \times 10^{7}$ Monte Carlo steps. After that, we calculate the dual self-energy and polarization operator diagrammatically and perform only the inner self-consistency loop in order to obtain the dressed Green function and renormalized interaction using the Dyson equation. For this purpose, we use a momentum grid with a maximum linear mesh size of $N_{k}=128$, with the number of fermionic and bosonic Matsubara frequencies being $N_{i \omega}=256$ and $N_{i \Omega}=64$, respectively. The expression for the lattice self-energy of the DB approach coincides with the one of the DF theory in Eq. (D8). The lattice polarization function can be found using a similar expression [224]:

$\Pi\left(\mathbf{q}, i \Omega_{n}\right)=\frac{\tilde{\Pi}\left(\mathbf{q}, i \Omega_{n}\right)}{1+\mathcal{W}\left(i \Omega_{n}\right) \tilde{\Pi}\left(\mathbf{q}, i \Omega_{n}\right)}+\Pi^{\mathrm{EDMFT}}\left(i \Omega_{n}\right)$.

The fully self-consistent DB calculations can be performed as follows. To obtain the fermionic hybridization of the effective impurity problem, we use the outer selfconsistency condition that equates the local part of the lattice Green function and local impurity Green function $\sum_{\mathbf{k}} G_{\mathbf{k} \omega \sigma}=g_{\omega \sigma}$. Regarding the bosonic hybridization function, there is no clear method for how this quantity has to be determined. Here, we investigate two different self-consistency schemes that fix the bosonic hybridization. For the X-self-consistent (Xsc) result, the local part of the lattice susceptibility is equated to the corresponding local susceptibility of the impurity problem $\sum_{\mathbf{q}} X_{\Omega}^{\varsigma}=\chi_{\Omega}^{\varsigma}$. The other self-consistency can be imposed on a renormalized (screened) interaction (Wsc) $\sum_{\mathbf{q}} W_{\Omega}^{\varsigma}=w_{\Omega}^{\varsigma}$. The renormalized interaction $W$ of the lattice problem can be defined as

$$
W^{\varsigma-1}\left(\mathbf{q}, i \Omega_{n}\right)=U^{\varsigma-1}-\Pi^{\varsigma}\left(\mathbf{q}, i \Omega_{n}\right),
$$

where $U^{\mathrm{ch} / \mathrm{sp}}= \pm U / 2$ [225]. The EDMFT renormalized interaction can be obtained by neglecting the dual contribution to the polarization operator in Eq. (D11), so that $\Pi^{\varsigma}\left(\mathbf{q}, i \Omega_{n}\right)=\Pi^{\mathrm{EDMFT}}\left(i \Omega_{n}\right)$. The renormalized interaction of the impurity problem can be found as

$w^{\varsigma^{-1}}\left(i \Omega_{n}\right)=\left(U^{\varsigma}+Y^{\varsigma}\left(i \Omega_{n}\right)\right)^{-1}-\Pi^{\varsigma \operatorname{EDMFT}}\left(i \Omega_{n}\right)$,

where $Y^{\varsigma}\left(i \Omega_{n}\right)$ is the bosonic hybridization function. Corresponding results are shown in Figs. 36 and 37. We note, as well, that the comparisons between self-consistent DB and self-consistent DF schemes are in good agreement, but they differ from the exact result. As we point out in the main text, the single-shot DB approach correctly reproduces exact DiagMC results at almost all temperatures. Surprisingly, we observe that the Xsc DB calculations strongly deviate from the exact result presented in both figures. At the same time, we find that the Wsc DB result for the self-energy agrees with DiagMC calculations even better than the single-shot DB one. However, two-particle quantities, such as the lattice susceptibility and double occupancy, get worse when the self-consistency on the renormalized interaction is utilized. This observation can be explained by the fact that the considered bosonic selfconsistencies cannot fix all desired single- and two-particle quantities at the same time. Therefore, the question of a good self-consistency for the bosonic hybridization function remains open.

\section{Other approximations a. TPSC and TPSC+ i. TPSC}

The two-particle self-consistent (TPSC) [93,94,258] approach is a nonperturbative approach that is only valid from weak to intermediate interaction strengths; hence, it does not describe the Mott transition. Nevertheless, there are a large number of physical phenomena that it allows us to study with an accuracy comparable to other numerically exact methods. In particular, in two dimensions, it describes the entry into the renormalized classical regime of 

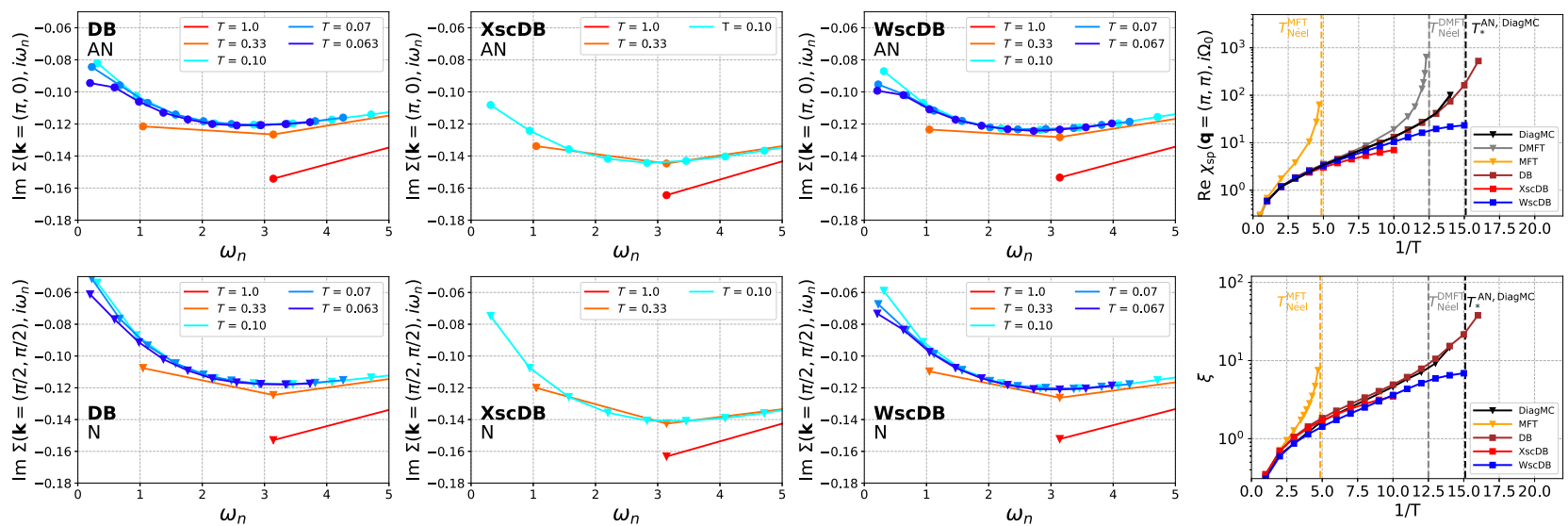

FIG. 36. Results from alternative DB schemes—single-shot (DB), self-consistent (XscDB), and W-self-consistent (WscDB)—for various quantities.

antiferromagnetic fluctuations. There are no adjustable parameters as opposed to, for example, self-consistent renormalized spin-fluctuation theory. It satisfies conservation laws for total spin and total charge, the Mermin-Wagner theorem, and the Pauli principle in the form $\left\langle n_{\sigma}^{2}\right\rangle=\left\langle n_{\sigma}\right\rangle$, as well as the local spin and local charge sum rules. The two local sum rules, in addition to the ansatz that the renormalized irreducible spin interaction vertex is given by $U_{\text {sp }}\left\langle n_{\uparrow}\right\rangle\left\langle n_{\downarrow}\right\rangle=$ $U\left\langle n_{\uparrow} n_{\downarrow}\right\rangle$, suffice to obtain the irreducible vertices

$U_{\mathrm{sp}} \equiv \frac{\delta \Sigma_{\uparrow}^{(1)}}{\delta G_{\downarrow}^{(1)}}-\frac{\delta \Sigma_{\uparrow}^{(1)}}{\delta G_{\uparrow}^{(1)}} ; \quad U_{\mathrm{ch}} \equiv \frac{\delta \Sigma_{\uparrow}^{(1)}}{\delta G_{\downarrow}^{(1)}}+\frac{\delta \Sigma_{\uparrow}^{(1)}}{\delta G_{\uparrow}^{(1)}}$,

and then compute the spin and charge susceptibilities from

$$
\begin{aligned}
& \chi_{\mathrm{sp}}(q)=\frac{\chi^{(1)}(q)}{1-\frac{1}{2} U_{\mathrm{sp}} \chi^{(1)}(q)} ; \\
& \chi_{\mathrm{ch}}(q)=\frac{\chi^{(1)}(q)}{1+\frac{1}{2} U_{\mathrm{ch}} \chi^{(1)}(q)} .
\end{aligned}
$$

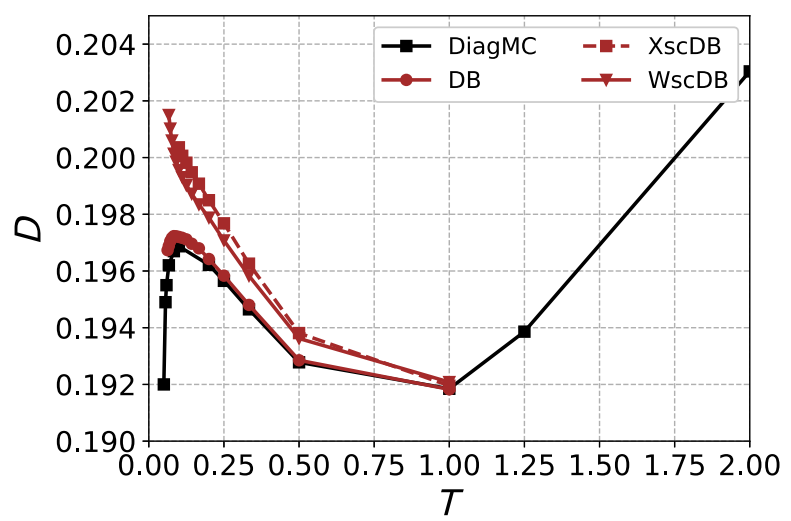

FIG. 37. Results from alternative DB schemes-single-shot (DB), self-consistent (XscDB), and W-self-consistent (WscDB) for the double occupancy compared to DiagMC.
The local spin and charge sum rules are given by $\operatorname{Tr} \chi_{\mathrm{sp}}(q)=$ $n-2\left\langle n_{\uparrow} n_{\downarrow}\right\rangle$ and $\operatorname{Tr} \chi_{\mathrm{ch}}(q)=n+2\left\langle n_{\uparrow} n_{\downarrow}\right\rangle-n^{2}$, respectively, and the trace is over the spin and momentum space. The above ansatz for $U_{\text {sp }}$ was inspired from Ref. [259,260] and was found independently in Ref. [261].

At the first-level approximation [hence, the superscript "(1)"] of TPSC, the irreducible particle-hole bubble diagram is obtained from

$$
\chi^{(1)}(q)=-\operatorname{Tr}\left[G^{(1)} G^{(1)}\right]=-\sum_{k} G_{0}(k) G_{0}(k+q),
$$

where the Green function $G_{\sigma}^{(1)}(k)$ includes a constant selfenergy $\Sigma_{\sigma}^{(1)}$ and renormalized chemical potential that lead to a noninteracting form $G_{0}(k)$. The two irreducible vertices suffice to find an improved self-energy [111] that does not assume Migdal's theorem and that takes into account rotational invariance and crossing symmetry,

$$
\begin{aligned}
\Sigma_{\sigma}^{(2)}(k)= & U n_{-\sigma} \\
& +\frac{U}{8} \sum_{q}\left[3 U_{\mathrm{sp}} \chi_{\mathrm{sp}}(q)+U_{\mathrm{ch}} \chi_{\mathrm{ch}}(q)\right] G_{\sigma}^{(1)}(k-q) .
\end{aligned}
$$

The consistency condition between one-particle and twoparticle properties (Galitskii-Migdal formula referred to in the main text),

$$
\frac{1}{2} \operatorname{Tr}\left[\Sigma^{(2)} G^{(1)}\right]=U\left\langle n_{\uparrow} n_{\downarrow}\right\rangle,
$$

serves as a guide for the domain of validity of TPSC. (Double occupancy obtained from sum rules on spin and charge equals that obtained from the self-energy and the Green function. When $\frac{1}{2} \operatorname{Tr}\left[\Sigma^{(2)} G^{(2)}\right]$ starts to deviate from 
the above, the method starts to fail.) See Refs. [34,262] for detailed comparisons with QMC calculations and other approaches and discussions of the physics. Reference [31] reviews the work related to the pseudogap and superconductivity up to 2005, including detailed comparisons with quantum cluster approaches in the regime of validity that overlaps with TPSC (intermediate coupling). A pedagogical review of the results and derivations appears in Ref. [35].

TPSC numerical results in the present paper have been obtained on a $256 \times 256$ lattice and with $2 \times 1024$ Matsubara frequencies with tails treated analytically. Because of the self-energy iterations, we use a $128 \times 128$ lattice and $2 \times 512$ Matsubara frequencies in TPSC+ calculations, so the sufficient precision can be obtained using moderate computational resources, as in TPSC.

\section{ii. TPSC+}

One of the main limitations of TPSC is that, even for weak to intermediate interaction strengths, it is not valid deep in the renormalized classical regime [258]. For example, for the half-filled Hubbard model with only nearest-neighbor hopping, one finds that $U_{\text {sp }}$ tends to zero as the temperature goes to zero, contrary to the fact that $U_{\mathrm{sp}}$ and the site double occupancy $\left\langle n_{\uparrow} n_{\downarrow}\right\rangle=\left(U_{\text {sp }} / U\right)\left\langle n_{\uparrow}\right\rangle\left\langle n_{\downarrow}\right\rangle$ must saturate to a finite value as $T \rightarrow 0$ due to virtual states.

To remedy this problem, an improved TPSC has recently been proposed [36]. We refer to this extension as TPSC+. It is based on an extension of the ideas of Kadanoff and Martin [263] who treated the normal state of superconductors in such a way that it connects smoothly to the superconducting state described by the BCS equation. The key idea is that the pair susceptibility takes an asymmetric form $\chi(q)=-\operatorname{Tr}\left[G(k) G_{0}(q-k)\right]$. It is called the pairing approximation or $G G_{0}$ theory in this context, and it has been extensively used by Levin's group to study pairing pseudogaps and related phenomena [264,265].

We apply that idea to the repulsive Hubbard model and use the asymmetric form in the particle-hole bubble,

$\tilde{\chi}^{(2)}=-\frac{1}{2} \operatorname{Tr}\left[\tilde{G}^{(2)}(k+q) G^{(1)}(k)+\tilde{G}^{(2)}(k-q) G^{(1)}(k)\right]$,

where the self-energy $\tilde{\Sigma}^{(2)}$ entering $\tilde{G}^{(2)}$ has the same form as Eq. (D16): Contrary to the original Kadanoff-Martin approach, the irreducible vertices are computed from the same sum rules and ansatz, but the susceptibilities in Eq. (D14) are obtained from $\tilde{\chi}^{(2)}$ instead of from $\chi^{(1)}$. The tilde symbol () indicates that $\tilde{\Sigma}^{(2)}, \tilde{G}^{(2)}$, and $\tilde{\chi}^{(2)}$ selfconsistently depend on each other. Note that the trace also includes the spin.
The advantages of this approach are as follows. (a) The generalized Stoner criterion for the phase transition temperature $T_{N}$ becomes identical to the mean-field gap equation in the antiferromagnetic state, with the interaction vertex reduced from the bare $U$ to $U_{\text {sp }}$ [36]. (b) The MerminWagner theorem and the Pauli principle are satisfied. Analytical arguments that demonstrate these results proceed in a manner analogous to those in TPSC. (c) One- and two-particle properties are consistent in the sense that $\frac{1}{2} \operatorname{Tr}\left[\tilde{\Sigma}^{(2)} \tilde{G}^{(2)}\right]=U\left\langle n_{\uparrow} n_{\downarrow}\right\rangle$ is satisfied exactly with double occupancy equal to that obtained from the local spin and charge sum rules. (d) Analytical arguments analogous to those in TPSC show that there is a pseudogap in two dimensions that is a precursor to the antiferromagnetically ordered state at zero temperature. Because the susceptibility dressed by the self-energy remains finite at zero temperature, renormalized $U_{\text {sp }}$ remains finite while pushing $T_{N}$ to zero. Furthermore, at zero temperature, the size of the pseudogap $\Delta_{\text {pseudogap }}(0)$ becomes equal to the finite magnetic gap $\Delta(0)$.

On the downside, the susceptibilities at zero wave vector do not vanish at finite frequency, as conservation laws say they should. However, the values of the zero-wave-vector susceptibilities at finite frequency are generally small. Finally, the $f$-sum rule is also slightly violated.

Heuristically, another explanation of the approach comes from the expected cancellations between vertices and selfenergy. Consider the Bethe-Salpeter equation for the fourpoint function (susceptibilities),

$$
\frac{\delta G}{\delta \phi}=-G \frac{\delta G^{-1}}{\delta \phi} G
$$

where we have used the identity $G G^{-1}=\delta(1-2)$. Suppose a quasiparticle picture applies, namely, $G=Z G_{0}$, where $Z$ is the quasiparticle weight.

In some approximation, the $Z^{-1}$ from the vertices $\delta G^{-1} / \delta \phi$ cancels $Z$ from one of the Green functions. In some sense, $U_{\text {sp }}$ and $U_{\text {ch }}$ account for the average momentum-space screening of the interaction vertices, while $Z^{-1}$ accounts for their frequency dependence.

Numerical results for TPSC+ in the present paper have been obtained on a $128 \times 128$ lattice with $2 \times 512$ Matsubara frequencies. Fast Fourier transforms with a uniform grid for imaginary time $\tau$ are used in the selfconsistent calculations of $\tilde{\Sigma}_{2 \mathrm{PT}}$ and $\tilde{\chi}_{2 \mathrm{PT}}$.

Eventually, we would like to comment on result from a TPSC+ variety, which utilizes the full Green function $G$ instead of $G_{0}$ and is therefore coined TPSC + GG. In Fig. 35, we see that there is very good agreement of the TPSC + GG with DГA for the spin susceptibility and correlation length. Figure 38 now also shows that at low $T$, 

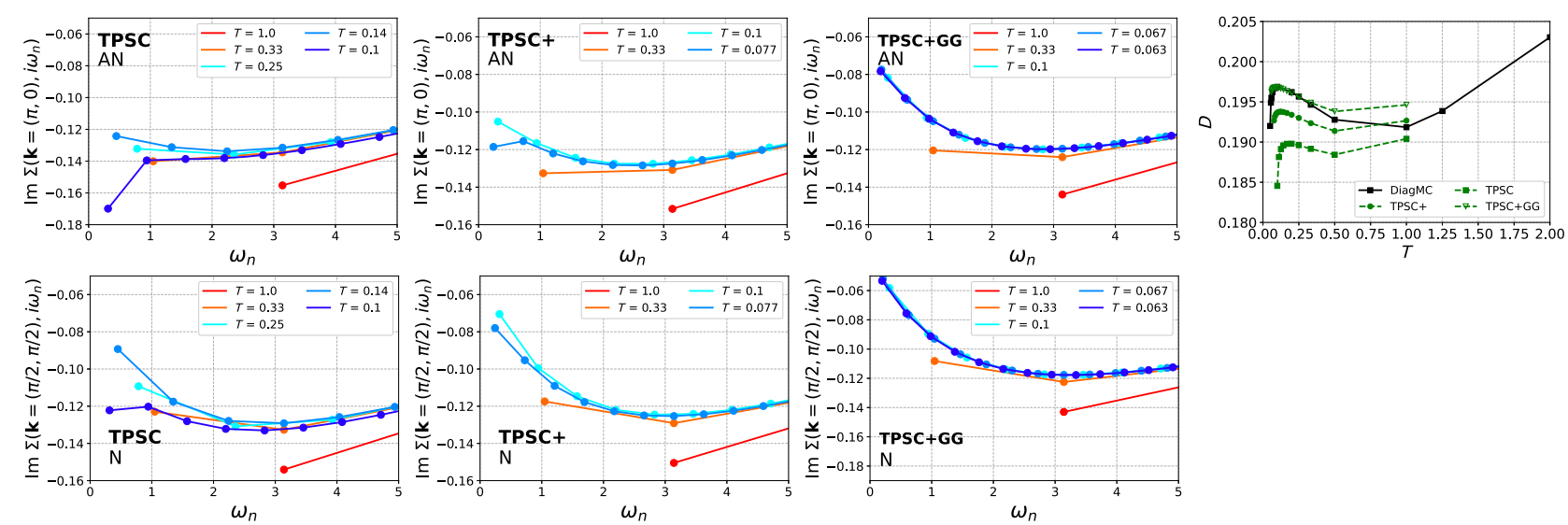

FIG. 38. Results from TPSC, TPSC+, and TPSC + GG.

the double occupancy agrees well with the benchmark, although it does not open the pseudogap.

\section{b. fRG}

The characteristic scale-dependent behavior of numerous strongly correlated electron systems can be treated in a flexible and unbiased way by the functional renormalization group (fRG); see Refs. [37,266-268] for a review (see also Refs. [269-272] for the context of the pseudogap). Its starting point is an exact functional flow equation, which yields the gradual evolution from a microscopic model action to the final effective action as a function of a continuously decreasing energy scale. Expanding in powers of the fields, one obtains an exact hierarchy of flow equations for the $n$-particle irreducible vertex functions, which, in practical implementations, is truncated at the two-particle level. Neglecting the renormalization of three- and higher-order particle vertices yields approximate one-loop flow equations for the self-energy and two-particle vertex [273].

The underlying approximations are devised for the weak to moderate coupling regimes. A substantial improvement with respect to previous fRG-based computation schemes relies on an efficient parametrization of the two-particle vertex, where we combine the so-called "truncated unity" fRG [274-276] using the channel decomposition in
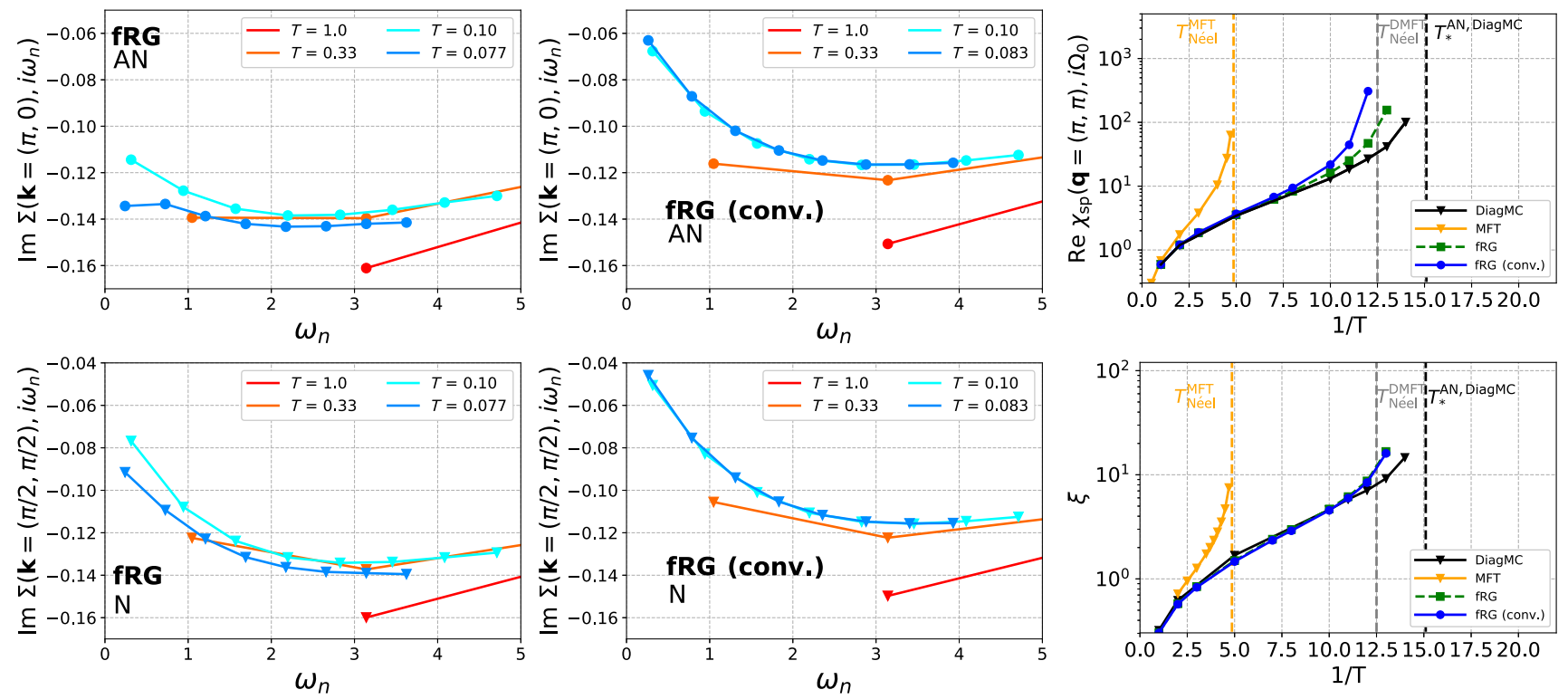

FIG. 39. Comparison of the imaginary parts of the self-energies at the antinode (upper panel) and node (lower panel) as a function of Matsubara frequencies calculated by using the scale derivative of the Schwinger-Dyson equation (fRG, left panels, presented in the main text) and by the conventional self-energy flow [fRG (conv.), middle panels]. In contrast to the results shown in Figs. 10 and 11 , the reduced renormalization of the self-energy in fRG (conv.) leads to an enhanced spin susceptibility $\operatorname{Re} \chi_{\text {sp }}\left(\mathbf{q}=(\pi, \pi)\right.$, i $\left.\Omega_{0}\right)$ (right upper panel) and correlation length $\xi$ (lower right panel). The self-energy implementation in the conventional flow does not capture the pseudogap opening. 
conjunction with a form-factor expansion for the momentum dependence with the full frequency treatment [277], which includes the high-frequency asymptotics [107,235]. Here, we use the Katanin replacement [278] in the flow equation for the two-particle vertex as a first step towards the multiloop extension of the fRG, which would allow us to sum up all the diagrams of the parquet approximation with their exact weight $[57,150]$. In order to account for the formfactor truncation, the self-energy flow is determined by the scale derivative of the Schwinger-Dyson equation [58,59] replacing the conventional one-loop flow equation (in Fig. 39). We refer to Refs. $[58,59,150]$ for the details of the algorithmic implementation and, in particular, also for the postprocessed computation of the susceptibilities from the flowing vertex and self-energy; the technical parameters are reported in Table II. The double occupancy is calculated from two-particle quantities according to

$$
D=\frac{1}{2 \beta} \sum_{\mathbf{q}, \Omega_{n}}\left[\chi_{\mathrm{ch}}\left(\mathbf{q}, i \Omega_{n}\right)-\chi_{\mathrm{sp}}\left(\mathbf{q}, i \Omega_{n}\right)\right]+\frac{n^{2}}{4} .
$$

We note that, also taking into account multiloop corrections, these algorithmic advancements have been shown to bring the fRG for interacting fermions on 2D lattices to a quantitatively reliable level, in particular, recovering the PA $[58,150]$.

\section{c. PA}

The parquet approach is a diagrammatic scheme first introduced by DeDominicis and Martin in 1964 [38]. It relies on the classification of diagrams contributing to the full one-particle irreducible two-particle vertex in terms of their two-particle reducibility. These diagrams can be either fully two-particle irreducible or two-particle reducible in one of three channels $[39,279,280]$. The method is unbiased with respect to the channels and exact if using the exact, fully two-particle irreducible vertex as an input.

The parquet approximation (PA) applied here consists in setting this fully two-particle irreducible vertex to the bare Hubbard $U$, i.e., its lowest-order contribution, which is correct only up to fourth order in perturbation theory. The PA has previously been used to study several condensedmatter problems, including the Hubbard model [210,211,281-285]. The results presented here have been obtained with the TUPS [62] implementation, which relies on the method of truncated unities $[61,276]$ and vertex asymptotics $[107,286]$ in order to represent vertex functions, as well as a previously developed parallelization scheme in order to perform computations efficiently [282,283].

All results are converged in the number of discrete lattice momenta $\left(N_{q}\right)$ and positive fermionic Matsubara frequencies $\left(N_{f+}\right)$ used. Concretely, we use linear mesh sizes of
TABLE II. Technical parameters for the fRG calculations for all temperatures, performed with a smooth frequency cutoff. Here, $n$ is the number of positive fermionic frequencies that determine the parametrization of the two-particle vertex (see Ref. [150] for the definitions). The rest function contains $(4 n+1) \times(2 n) \times(2 n)$, the $K_{2}$-function and the fermion-boson vertex $(4 n+1) \times(2 n)$, the $K_{1}$-function $(128 n+1)$, and the self-energy $(8 n)$ frequencies. The fermionic momentum dependence of the vertices and response functions is accounted for by a form-factor expansion, where we consider only the local $s$-wave contribution since, at half-filling, the physics is dominated by antiferromagnetic fluctuations. The remaining momentum dependence of the vertices, the response functions, and the self-energy is calculated on $\left(k_{x} \times k_{x}\right)$ equally spaced momentum patches, while the Green functions and their summation in the particle-hole and particleparticle excitation are calculated on a $\left(p_{x} \times p_{x}\right)$ grid. The grid for the vertices and response functions is refined around $\mathbf{q}=(\pi, \pi)$, adding $\left(k_{x}^{\text {refine }} \times k_{x}^{\text {refine }}\right)$ patches from the $\left(p_{x} \times p_{x}\right)$ grid, accounting for 216 additional patches with respect to the ones already included in the $\left(k_{x} \times k_{x}\right)$ grid.

\begin{tabular}{llrcr}
\hline \hline $1 / T$ & $n$ & $k_{x}$ & $k_{x}^{\text {refine }}$ & $p_{x}$ \\
\hline 1 & 4 & 8 & 15 & 40 \\
2 & 4 & 8 & 15 & 40 \\
3 & 4 & 8 & 15 & 40 \\
5 & 6 & 12 & 15 & 60 \\
7 & 8 & 16 & 15 & 80 \\
8 & 8 & 16 & 15 & 80 \\
10 & 8 & 16 & 15 & 80 \\
11 & 8 & 16 & 15 & 80 \\
12 & 8 & 16 & 15 & 80 \\
13 & 8 & 20 & 15 & 100 \\
\hline \hline
\end{tabular}

$N_{q}=48$ and $N_{f+}=40$ at $\beta t=16$ and fewer frequencies and momenta for higher temperatures. The results for $\beta t=$ 20 have been extrapolated to an infinite grid size. The number of basis functions $\left(N_{\mathrm{FF}}\right)$ inherent to the truncated unity method [61] is set to $N_{\mathrm{FF}}=9$ for all calculations. As has been shown in Ref. [61], it is sufficient to reproduce the full basis results for $\beta t \leq 5$, but it constitutes an additional approximation for lower temperatures. This approximation is quite likely responsible for the PA underestimation of the AFM susceptibility (and, as a consequence, the pseudogap temperature) in comparison with the benchmark. The AFM susceptibility is also underestimated in the full parquet DГА scheme, when the number of basis functions $N_{\mathrm{FF}}$ is limited to $N_{\mathrm{FF}} \leq 9$, as has been shown in Ref. [287]. A very promising new computational scheme has recently been proposed [288], which may allow for significant reduction of frequency box sizes and thus make computations with more basis functions $\left(N_{\mathrm{FF}}>9\right)$ feasible. The double occupancy was calculated using Eq. (8). Note that in the $\mathrm{PA}$, the sum rule relating the double occupancy obtained from one-particle and two-particle quantities, 


$$
\begin{aligned}
D & =\frac{1}{2 \beta} \sum_{\mathbf{q}, \Omega_{n}}\left[\chi_{\mathrm{ch}}\left(\mathbf{q}, i \Omega_{n}\right)-\chi_{\mathrm{sp}}\left(\mathbf{q}, i \Omega_{n}\right)\right]+\frac{n^{2}}{4} \\
& =\frac{1}{\beta} \sum_{\mathbf{k}, \omega_{n}} \Sigma\left(\mathbf{k}, i \omega_{n}\right) G\left(\mathbf{k}, i \omega_{n}\right),
\end{aligned}
$$

is fulfilled, by construction. The PA also fulfills $[60,62]$ the Mermin-Wagner theorem [21,22].

[1] J. Hubbard, Electron Correlations in Narrow Energy Bands, Proc. R. Soc. A 276, 238 (1963).

[2] J. Hubbard, Electron Correlations in Narrow Energy Bands. III. An Improved Solution, Proc R. Soc. A 281, 401 (1964).

[3] M. C. Gutzwiller, Effect of Correlation on the Ferromagnetism of Transition Metals, Phys. Rev. Lett. 10, 159 (1963).

[4] J. Kanamori, Electron Correlation and Ferromagnetism of Transition Metals, Prog. Theor. Phys. 30, 275 (1963).

[5] D. Jaksch and P. Zoller, The Cold Atom Hubbard Toolbox, Ann. Phys. (N.Y.) 315, 52 (2005), Special Issue.

[6] I. Bloch, Ultracold Quantum Gases in Optical Lattices, Nat. Phys. 1, 23 (2005).

[7] M. Lewenstein, A. Sanpera, V. Ahufinger, B. Damski, A. Sen(De), and U. Sen, Ultracold Atomic Gases in Optical Lattices: Mimicking Condensed Matter Physics and Beyond, Adv. Phys. 56, 243 (2007).

[8] F. H. L. Essler, H. Frahm, F. Göhmann, A. Klümper, and V. Korepin, The One-Dimensional Hubbard Model (Cambridge University Press, Cambridge, England, 2005).

[9] W. Metzner and D. Vollhardt, Correlated Lattice Fermions in $d=\infty$ Dimensions, Phys. Rev. Lett. 62, 324 (1989).

[10] A. Georges and G. Kotliar, Hubbard Model in Infinite Dimensions, Phys. Rev. B 45, 6479 (1992).

[11] M. Jarrell, Hubbard Model in Infinite Dimensions: A Quantum Monte Carlo Study, Phys. Rev. Lett. 69, 168 (1992).

[12] B. Keimer, S. A. Kivelson, M. R. Norman, S. Uchida, and J. Zaanen, From Quantum Matter to High-Temperature Superconductivity in Copper Oxides, Nature (London) 518, 179 (2015).

[13] T. Timusk and B. Statt, The Pseudogap in HighTemperature Superconductors: An Experimental Survey, Rep. Prog. Phys. 62, 61 (1999).

[14] M. R. Norman, D. Pines, and C. Kallin, The Pseudogap: Friend or Foe of High $T_{c}$ ?, Adv. Phys. 54, 715 (2005).

[15] P. A. Lee, N. Nagaosa, and X.-G. Wen, Doping a Mott Insulator: Physics of High-Temperature Superconductivity, Rev. Mod. Phys. 78, 17 (2006).

[16] J.P. F. LeBlanc et al., Solutions of the Two-Dimensional Hubbard Model: Benchmarks and Results from a Wide Range of Numerical Algorithms, Phys. Rev. X 5, 041041 (2015).

[17] M. Motta et al., Towards the Solution of the ManyElectron Problem in Real Materials: Equation of State of the Hydrogen Chain with State-of-the-Art Many-Body Methods, Phys. Rev. X 7, 031059 (2017).
[18] K. T. Williams et al., Direct Comparison of Many-Body Methods for Realistic Electronic Hamiltonians, Phys. Rev. X 10, 011041 (2020).

[19] A. Neronov, Introduction to Multi-messenger Astronomy, J. Phys. Conf. Ser. 1263, 012001 (2019).

[20] J. C. Slater, Magnetic Effects and the Hartree-Fock Equation, Phys. Rev. 82, 538 (1951).

[21] N. D. Mermin and H. Wagner, Absence of Ferromagnetism or Antiferromagnetism in One- or Two-Dimensional Isotropic Heisenberg Models, Phys. Rev. Lett. 17, 1307 (1966).

[22] P. C. Hohenberg, Existence of Long-Range Order in One and Two Dimensions, Phys. Rev. 158, 383 (1967).

[23] R. Blankenbecler, D. J. Scalapino, and R. L. Sugar, Monte Carlo Calculations of Coupled Boson-Fermion Systems. I, Phys. Rev. D 24, 2278 (1981).

[24] N. V. Prokof'ev and B. V. Svistunov, Polaron Problem by Diagrammatic Quantum Monte Carlo, Phys. Rev. Lett. 81, 2514 (1998).

[25] R. Rossi, Determinant Diagrammatic Monte Carlo Algorithm in the Thermodynamic Limit, Phys. Rev. Lett. 119, 045701 (2017).

[26] F. Šimkovic and E. Kozik, Determinant Monte Carlo for Irreducible Feynman Diagrams in the Strongly Correlated Regime, Phys. Rev. B 100, 121102(R) (2019).

[27] A. Moutenet, W. Wu, and M. Ferrero, Determinant Monte Carlo Algorithms for Dynamical Quantities in Fermionic Systems, Phys. Rev. B 97, 085117 (2018).

[28] A. Georges, G. Kotliar, W. Krauth, and M. J. Rozenberg, Dynamical Mean-Field Theory of Strongly Correlated Fermion Systems and the Limit of Infinite Dimensions, Rev. Mod. Phys. 68, 13 (1996).

[29] T. A. Maier, M. Jarrell, T. Pruschke, and M. Hettler, Quantum Cluster Theories, Rev. Mod. Phys. 77, 1027 (2005).

[30] G. Kotliar, S. Y. Savrasov, K. Haule, V. S. Oudovenko, O. Parcollet, and C. A. Marianetti, Electronic Structure Calculations with Dynamical Mean-Field Theory, Rev. Mod. Phys. 78, 865 (2006).

[31] A. M. S. Tremblay, B. Kyung, and D. Sénéchal, Pseudogap and High-Temperature Superconductivity from Weak to Strong Coupling. Towards a Quantitative Theory, Low Temp. Phys. 32, 424 (2006).

[32] A. I. Lichtenstein and M. I. Katsnelson, Antiferromagnetism and d-wave Superconductivity in Cuprates: A Cluster Dynamical Mean-Field Theory, Phys. Rev. B 62, R9283 (2000).

[33] G. Rohringer, H. Hafermann, A. Toschi, A. A. Katanin, A. E. Antipov, M. I. Katsnelson, A. I. Lichtenstein, A. N. Rubtsov, and K. Held, Diagrammatic Routes to Nonlocal Correlations Beyond Dynamical Mean Field Theory, Rev. Mod. Phys. 90, 025003 (2018).

[34] Y. M. Vilk and A.-M. S. Tremblay, Non-Perturbative ManyBody Approach to the Hubbard Model and Single-Particle Pseudogap, J. Phys. I (France) 7, 1309 (1997).

[35] A. M. S. Tremblay, Two-Particle-Self-Consistent Approach for the Hubbard Model, in Strongly Correlated Systems: Theoretical Methods, edited by F. Mancini and A. Avella (Springer, New York, 2011), Chap. 13, pp. $409-455$. 
[36] Y. Wang, Y. M. Vilk, and A.-M. S. Tremblay, An Improved Two-Particle Self-Consistent Approach (to be published).

[37] W. Metzner, M. Salmhofer, C. Honerkamp, V. Meden, and K. Schönhammer, Functional Renormalization Group Approach to Correlated Fermion Systems, Rev. Mod. Phys. 84, 299 (2012).

[38] C. De Dominicis and P. C. Martin, Stationary Entropy Principle and Renormalization in Normal and Superfluid Systems. II. Diagrammatic Formulation, J. Math. Phys. (N.Y.) 5, 31 (1964).

[39] N. E. Bickers, Theoretical Methods for Strongly Correlated Electrons, edited by D. Sénéchal, C. Bourbonnais, and A.-M.S. Tremblay (Springer-Verlag, New York, Berlin, Heidelbert, 2004), Chap. 6, pp. 237-296.

[40] S. R. White, Minimally Entangled Typical Quantum States at Finite Temperature, Phys. Rev. Lett. 102, 190601 (2009).

[41] E. M. Stoudenmire and S. R. White, Minimally Entangled Typical Thermal State Algorithms, New J. Phys. 12, 055026 (2010).

[42] B. Bruognolo, J. von Delft, and A. Weichselbaum, Symmetric Minimally Entangled Typical Thermal States, Phys. Rev. B 92, 115105 (2015).

[43] A. Wietek, Y.-Y. He, S. R. White, A. Georges, and E. M. Stoudenmire, Stripes, Antiferromagnetism, and the Pseudogap in the Doped Hubbard Model at Finite Temperature, arXiv:2009.10736 [Phys. Rev X (to be published)].

[44] A. Wietek, R. Rossi, F. Šimkovic IV, M. Klett, P. Hansmann, M. Ferrero, E. M. Stoudenmire, T. Schäfer, and A. Georges, Mott Insulating States with Competing Orders in the Triangular Lattice Hubbard Model, arXiv: 2102.12904.

[45] See Supplemental Material at http://link.aps.org/ supplemental/10.1103/PhysRevX.11.011058 for the numerical data used in the figures of the main text, which includes Ref. [46].

[46] T. Schäfer et al., Tracking the Footprints of Spin Fluctuations: A Multi-Method, Multi-Messenger Study of the TwoDimensional Hubbard Model, arXiv:2006.10769.

[47] T. Schäfer, F. Geles, D. Rost, G. Rohringer, E. Arrigoni, K. Held, N. Blümer, M. Aichhorn, and A. Toschi, Fate of the False Mott-Hubbard Transition in Two Dimensions, Phys. Rev. B 91, 125109 (2015).

[48] F. Šimkovic, J. P. F. LeBlanc, A. J. Kim, Youjin Deng, N. V. Prokof'ev, B. V. Svistunov, and E. Kozik, Extended Crossover from a Fermi Liquid to a Quasiantiferromagnet in the Half-Filled 2D Hubbard Model, Phys. Rev. Lett. 124, 017003 (2020).

[49] A. J. Kim, F. Simkovic, and E. Kozik, Spin and Charge Correlations across the Metal-to-Insulator Crossover in the Half-Filled 2D Hubbard Model, Phys. Rev. Lett. 124, 117602 (2020).

[50] A. Tanaka, Metal-Insulator Transition in the TwoDimensional Hubbard Model: Dual Fermion Approach with Lanczos Exact Diagonalization, Phys. Rev. B 99, 205133 (2019).

[51] G. Rohringer and A. Toschi, Impact of Nonlocal Correlations over Different Energy Scales: A Dynamical Vertex Approximation Study, Phys. Rev. B 94, 125144 (2016).
[52] T. Schäfer, A. Toschi, and K. Held, Dynamical Vertex Approximation for the Two-Dimensional Hubbard Model, J. Magn. Magn. Mater. 400, 107 (2016).

[53] A. A. Katanin, A. Toschi, and K. Held, Comparing Pertinent Effects of Antiferromagnetic Fluctuations in the Two- and Three-Dimensional Hubbard Model, Phys. Rev. B 80, 075104 (2009).

[54] G. Rohringer and A. Toschi (private communication).

[55] T. Baier, E. Bick, and C. Wetterich, Temperature Dependence of Antiferromagnetic Order in the Hubbard Model, Phys. Rev. B 70, 125111 (2004).

[56] F. B. Kugler and J. von Delft, Multiloop Functional Renormalization Group that Sums Up All Parquet Diagrams, Phys. Rev. Lett. 120, 057403 (2018).

[57] F. B. Kugler and J. von Delft, Multiloop Functional Renormalization Group for General Models, Phys. Rev. B 97, 035162 (2018).

[58] C. Hille, F. B. Kugler, C. J. Eckhardt, Y.-Y. He, A. Kauch, C. Honerkamp, A. Toschi, and S. Andergassen, Quantitative Functional Renormalization Group Description of the Two-Dimensional Hubbard Model, Phys. Rev. Research 2, 033372 (2020).

[59] C. Hille, D. Rohe, C. Honerkamp, and S. Andergassen, Pseudogap Opening in the Two-Dimensional Hubbard Model: A Functional Renormalization Group Analysis, Phys. Rev. Research 2, 033068 (2020).

[60] N.E. Bickers and D. J. Scalapino, Critical Behavior of Electronic Parquet Solutions, Phys. Rev. B 46, 8050 (1992).

[61] C. J. Eckhardt, G. A. H. Schober, J. Ehrlich, and C. Honerkamp, Truncated-Unity Parquet Equations: Application to the Repulsive Hubbard Model, Phys. Rev. B 98, 075143 (2018).

[62] C. J. Eckhardt, C. Honerkamp, K. Held, and A. Kauch, Truncated Unity Parquet Solver, Phys. Rev. B 101, 155104 (2020).

[63] D. Rost, E. V. Gorelik, F. Assaad, and N. Blümer, Momentum-Dependent Pseudogaps in the Half-Filled Two-Dimensional Hubbard Model, Phys. Rev. B 86, 155109 (2012).

[64] D. Rost and N. Blümer, Deciding the Fate of the False Mott Transition in Two Dimensions by Exact Quantum Monte Carlo Methods, J. Phys. Conf. Ser. 640, 012047 (2015).

[65] G. Sangiovanni, A. Toschi, E. Koch, K. Held, M. Capone, C. Castellani, O. Gunnarsson, S.-K. Mo, J. W. Allen, H.-D. Kim, A. Sekiyama, A. Yamasaki, S. Suga, and P. Metcalf, Static versus Dynamical Mean-Field Theory of Mott Antiferromagnets, Phys. Rev. B 73, 205121 (2006).

[66] G. Parisi, Statistical Field Theory (Perseus Books Group, New York, 1998).

[67] M. H. Hettler, A. N. Tahvildar-Zadeh, M. Jarrell, T. Pruschke, and H. R. Krishnamurthy, Nonlocal Dynamical Correlations of Strongly Interacting Electron Systems, Phys. Rev. B 58, R7475 (1998).

[68] M. H. Hettler, M. Mukherjee, M. Jarrell, and H. R. Krishnamurthy, Dynamical Cluster Approximation: Nonlocal Dynamics of Correlated Electron Systems, Phys. Rev. B 61, 12739 (2000). 
[69] K. Aryanpour, M. H. Hettler, and M. Jarrell, Analysis of the Dynamical Cluster Approximation for the Hubbard Model, Phys. Rev. B 65, 153102 (2002).

[70] G. Kotliar, S. Y. Savrasov, G. Pálsson, and G. Biroli, Cellular Dynamical Mean Field Approach to Strongly Correlated Systems, Phys. Rev. Lett. 87, 186401 (2001).

[71] M. Klett, N. Wentzell, T. Schäfer, F. Simkovic, O. Parcollet, S. Andergassen, and Philipp Hansmann, Real-Space Cluster Dynamical Mean-Field Theory: Center-Focused Extrapolation on the One- and Two Particle-Levels, Phys. Rev. Research 2, 033476 (2020).

[72] G. Rohringer, A. Toschi, A. Katanin, and K. Held, Critical Properties of the Half-Filled Hubbard Model in Three Dimensions, Phys. Rev. Lett. 107, 256402 (2011).

[73] E. Gull, P. Werner, X. Wang, M. Troyer, and A. J. Millis, Local Order and the Gapped Phase of the Hubbard Model: A Plaquette Dynamical Mean-Field Investigation, Europhys. Lett. 84, 37009 (2008).

[74] E. Gull, O. Parcollet, P. Werner, and A. J. Millis, Momentum-Sector-Selective Metal-Insulator Transition in the Eight-Site Dynamical Mean-Field Approximation to the Hubbard Model in Two Dimensions, Phys. Rev. B 80, 245102 (2009).

[75] E. Gull, M. Ferrero, O. Parcollet, A. Georges, and A. J. Millis, Momentum-Space Anisotropy and Pseudogaps: A Comparative Cluster Dynamical Mean-Field Analysis of the Doping-Driven Metal-Insulator Transition in the Two-Dimensional Hubbard Model, Phys. Rev. B 82, 155101 (2010).

[76] E. Gull, O. Parcollet, and A. J. Millis, Superconductivity and the Pseudogap in the Two-Dimensional Hubbard Model, Phys. Rev. Lett. 110, 216405 (2013).

[77] O. Parcollet, G. Biroli, and G. Kotliar, Cluster Dynamical Mean Field Analysis of the Mott Transition, Phys. Rev. Lett. 92, 226402 (2004).

[78] A. Macridin, M. Jarrell, T. Maier, P. R. C. Kent, and E. D'Azevedo, Pseudogap and Antiferromagnetic Correlations in the Hubbard Model, Phys. Rev. Lett. 97, 036401 (2006).

[79] D. Sénéchal and A.-M. S. Tremblay, Hot Spots and Pseudogaps for Hole- and Electron-Doped High-Temperature Superconductors, Phys. Rev. Lett. 92, 126401 (2004).

[80] K. Haule and G. Kotliar, Strongly Correlated Superconductivity: A Plaquette Dynamical Mean-Field Theory Study, Phys. Rev. B 76, 104509 (2007).

[81] H. Park, K. Haule, and G. Kotliar, Cluster Dynamical Mean Field Theory of the Mott Transition, Phys. Rev. Lett. 101, 186403 (2008).

[82] M. Ferrero, P. S. Cornaglia, L. De Leo, O. Parcollet, G. Kotliar, and A. Georges, Pseudogap Opening and Formation of Fermi Arcs as an Orbital-Selective Mott Transition in Momentum Space, Phys. Rev. B 80, 064501 (2009).

[83] M. Ferrero, P. S. Cornaglia, L. De Leo, O. Parcollet, G. Kotliar, and A. Georges, Valence Bond Dynamical Mean-Field Theory of Doped Mott Insulators with Nodal/Antinodal Differentiation, Europhys. Lett. 85, 57009 (2009).

[84] P. Werner, E. Gull, O. Parcollet, and A. J. Millis, Momentum-Selective Metal-Insulator Transition in the
Two-Dimensional Hubbard Model: An 8-Site Dynamical Cluster Approximation Study, Phys. Rev. B 80, 045120 (2009).

[85] G. Sordi, P. Sémon, K. Haule, and A.-M. S. Tremblay, Strong Coupling Superconductivity, Pseudogap, and Mott Transition, Phys. Rev. Lett. 108, 216401 (2012).

[86] G. Sordi, P. Sémon, K. Haule, and A.-M. S. Tremblay, Pseudogap Temperature as a Widom Line in Doped Mott Insulators, Sci. Rep. 2, 547 (2012).

[87] G. Sordi, P. Sémon, K. Haule, and A.-M. S. Tremblay, c-Axis Resistivity, Pseudogap, Superconductivity, and Widom Line in Doped Mott Insulators, Phys. Rev. B 87, 041101(R) (2013).

[88] O. Gunnarsson, T. Schäfer, J.P. F. LeBlanc, E. Gull, J. Merino, G. Sangiovanni, G. Rohringer, and A. Toschi, Fluctuation Diagnostics of the Electron Self-Energy: Origin of the Pseudogap Physics, Phys. Rev. Lett. 114, 236402 (2015).

[89] W. Wu, M. S. Scheurer, S. Chatterjee, S. Sachdev, A. Georges, and M. Ferrero, Pseudogap and Fermi-Surface Topology in the Two-Dimensional Hubbard Model, Phys. Rev. X 8, 021048 (2018).

[90] M. S. Scheurer, S. Chatterjee, W. Wu, M. Ferrero, A. Georges, and S. Sachdev, Topological Order in the Pseudogap Metal, Proc. Natl. Acad. Sci. U.S.A. 115, E3665 (2018).

[91] A. Reymbaut, S. Bergeron, R. Garioud, M. Thénault, M. Charlebois, P. Sémon, and A.-M. S. Tremblay, Pseudogap, van Hove Singularity, Maximum in Entropy, and Specific Heat for Hole-Doped Mott Insulators, Phys. Rev. Research 1, 023015 (2019).

[92] L. Fratino, P. Sémon, M. Charlebois, G. Sordi, and A.-M. S. Tremblay, Signatures of the Mott Transition in the Antiferromagnetic State of the Two-Dimensional Hubbard Model, Phys. Rev. B 95, 235109 (2017).

[93] Y. Vilk, Destruction of the Fermi Liquid by Spin Fluctuations in Two Dimensions, J. Phys. Chem. Solids 56, 1769 (1995).

[94] Y. M. Vilk and A.-M. S. Tremblay, Destruction of FermiLiquid Quasiparticles in Two Dimensions by Critical Fluctuations, Europhys. Lett. 33, 159 (1996).

[95] A. Georges and W. Krauth, Physical Properties of the Half-Filled Hubbard Model in Infinite Dimensions, Phys. Rev. B 48, 7167 (1993).

[96] F. Werner, O. Parcollet, A. Georges, and S. R. Hassan, Interaction-Induced Adiabatic Cooling and Antiferromagnetism of Cold Fermions in Optical Lattices, Phys. Rev. Lett. 95, 056401 (2005).

[97] A.-M. Daré, L. Raymond, G. Albinet, and A.-M. S. Tremblay, Interaction-Induced Adiabatic Cooling for Antiferromagnetism in Optical Lattices, Phys. Rev. B 76, 064402 (2007).

[98] S. Taie, R. Yamazaki, S. Sugawa, and Y. Takahashi, An SU(6) Mott Insulator of an Atomic Fermi Gas Realized by Large-Spin Pomeranchuk Cooling, Nat. Phys. 8, 825 (2012).

[99] V. M. Galitskii and A. B. Migdal, Application of Quantum Field Theoretical Methods to the Many-Body Problem, Sov. Phys. JETP 34, 139 (1958), http://www.jetp.ac.ru/cgibin/e/index/e/7/1/p96?a=list. 
[100] E. G. C. P. van Loon, F. Krien, H. Hafermann, E. A. Stepanov, A. I. Lichtenstein, and M. I. Katsnelson, Double Occupancy in Dynamical Mean-Field Theory and the Dual Boson Approach, Phys. Rev. B 93, 155162 (2016).

[101] S. Chakravarty, B. I. Halperin, and D. R. Nelson, Low-Temperature Behavior of Two-Dimensional Quantum Antiferromagnets, Phys. Rev. Lett. 60, 1057 (1988).

[102] S. Chakravarty, B. I. Halperin, and D. R. Nelson, TwoDimensional Quantum Heisenberg Antiferromagnet at Low Temperatures, Phys. Rev. B 39, 2344 (1989).

[103] K. Borejsza and N. Dupuis, Antiferromagnetism and SingleParticle Properties in the Two-Dimensional Half-Filled Hubbard Model: Slater vs. Mott-Heisenberg, Europhys. Lett. 63, 722 (2003).

[104] K. Borejsza and N. Dupuis, Antiferromagnetism and Single-Particle Properties in the Two-Dimensional Half-Filled Hubbard Model: A Nonlinear Sigma Model Approach, Phys. Rev. B 69, 085119 (2004).

[105] T. A. Maier, M. Jarrell, T. C. Schulthess, P. R. C. Kent, and J. B. White, Systematic Study of d-Wave Superconductivity in the 2D Repulsive Hubbard Model, Phys. Rev. Lett. 95, 237001 (2005).

[106] A. Toschi, A. A. Katanin, and K. Held, Dynamical Vertex Approximation-A Step beyond Dynamical Mean-Field Theory, Phys. Rev. B 75, 045118 (2007).

[107] N. Wentzell, G. Li, A. Tagliavini, C. Taranto, G. Rohringer, K. Held, A. Toschi, and S. Andergassen, High-Frequency Asymptotics of the Vertex Function: Diagrammatic Parametrization and Algorithmic Implementation, Phys. Rev. B 102, 085106 (2020).

[108] J. Kaufmann, P. Gunacker, and K. Held, Continuous-Time Quantum Monte Carlo Calculation of Multiorbital Vertex Asymptotics, Phys. Rev. B 96, 035114 (2017).

[109] A. Katanin, Improved Treatment of Fermion-Boson Vertices and Bethe-Salpeter Equations in Nonlocal Extensions of Dynamical Mean Field Theory, Phys. Rev. B 101, 035110 (2020).

[110] L. S. Ornstein and F. Zernike, Proc. R. Acad. Amsterdam 17, 793 (1914), https:/www.dwc.knaw.nl/DL/publications/ PU00012727.pdf.

[111] S. Moukouri, S. Allen, F. Lemay, B. Kyung, D. Poulin, Y. M. Vilk, and A.-M. S. Tremblay, Many-Body Theory versus Simulations for the Pseudogap in the Hubbard Model, Phys. Rev. B 61, 7887 (2000).

[112] T. A. Sedrakyan and A. V. Chubukov, Pseudogap in Underdoped Cuprates and Spin-Density-Wave Fluctuations, Phys. Rev. B 81, 174536 (2010).

[113] Y. M. Vilk, Shadow Features and Shadow Bands in the Paramagnetic State of Cuprate Superconductors, Phys. Rev. B 55, 3870 (1997).

[114] F. Lemay, Des Propriétés de l'état Normal du Modèle de Hubbard Bidimensionnel, Ph.D. thesis, Université de Sherbrooke, 2000, https://savoirs.usherbrooke.ca/bitstream/ handle/11143/4993/NQ67111.pdf.

[115] V. Zlatic, Temperature Dependence of the Spectral Properties for 2-D Hubbard Model with Anisotropic Hopping, Physica B (Amsterdam) 230, 1034 (1997).

[116] A. A. Katanin, A. P. Kampf, and V. Yu. Irkhin, Anomalous Self-Energy and Fermi Surface Quasisplitting in the
Vicinity of a Ferromagnetic Instability, Phys. Rev. B 71, 085105 (2005).

[117] E. Müller-Hartmann, The Hubbard Model at High Dimensions: Some Exact Results and Weak Coupling Theory, Z. Phys. B 76, 211 (1989).

[118] W. Ku and A. G. Eguiluz, Band-Gap Problem in Semiconductors Revisited: Effects of Core States and ManyBody Self-Consistency, Phys. Rev. Lett. 89, 126401 (2002).

[119] K. Delaney, P. García-González, A. Rubio, P. Rinke, and R.W. Godby, Comment on Band-Gap Problem in Semiconductors Revisited: Effects of Core States and ManyBody Self-Consistency, Phys. Rev. Lett. 93, 249701 (2004).

[120] W. Ku and A. G. Eguiluz, Ku and Eguiluz Reply, Phys. Rev. Lett. 93, 249702 (2004).

[121] N. Prokof'ev and B. Svistunov, Bold Diagrammatic Monte Carlo Technique: When the Sign Problem Is Welcome, Phys. Rev. Lett. 99, 250201 (2007).

[122] Y. Deng, E. Kozik, N. V. Prokof'ev, and B. V. Svistunov, Emergent BCS Regime of the Two-Dimensional Fermionic Hubbard Model: Ground-State Phase Diagram, Europhys. Lett. 110, 57001 (2015).

[123] E. Kozik, M. Ferrero, and A. Georges, Nonexistence of the Luttinger-Ward Functional and Misleading Convergence of Skeleton Diagrammatic Series for Hubbard-Like Models, Phys. Rev. Lett. 114, 156402 (2015).

[124] A. Stan, P. Romaniello, S. Rigamonti, L. Reining, and J. A. Berger, Unphysical and Physical Solutions in Many-Body Theories: From Weak to Strong Correlation, New J. Phys. 17, 093045 (2015).

[125] J. Vučičević, N. Wentzell, M. Ferrero, and O. Parcollet, Practical Consequences of the Luttinger-Ward Functional Multivaluedness for Cluster DMFT Methods, Phys. Rev. B 97, 125141 (2018).

[126] R. Rossi and F. Werner, Skeleton Series and Multivaluedness of the Self-Energy Functional in Zero Space-Time Dimensions, J. Phys. A 48, 485202 (2015).

[127] A. J. Kim and V. Sacksteder, Multivaluedness of the Luttinger-Ward Functional in the Fermionic and Bosonic System with Replicas, Phys. Rev. B 101, 115146 (2020).

[128] O. Gunnarsson, G. Rohringer, T. Schäfer, G. Sangiovanni, and A. Toschi, Breakdown of Traditional Many-Body Theories for Correlated Electrons, Phys. Rev. Lett. 119, 056402 (2017).

[129] T. Schäfer, G. Rohringer, O. Gunnarsson, S. Ciuchi, G. Sangiovanni, and A. Toschi, Divergent Precursors of the Mott-Hubbard Transition at the Two-Particle Level, Phys. Rev. Lett. 110, 246405 (2013).

[130] V. Janiš and V. Pokorný, Critical Metal-Insulator Transition and Divergence in a Two-Particle Irreducible Vertex in Disordered and Interacting Electron Systems, Phys. Rev. B 90, 045143 (2014).

[131] T. Schäfer, S. Ciuchi, M. Wallerberger, P. Thunström, O. Gunnarsson, G. Sangiovanni, G. Rohringer, and A. Toschi, Non-perturbative Landscape of the Mott-Hubbard Transition: Multiple Divergence Lines around the Critical Endpoint, Phys. Rev. B 94, 235108 (2016).

[132] P. Chalupa, P. Gunacker, T. Schäfer, K. Held, and A. Toschi, Divergences of the Irreducible Vertex Functions in Correlated Metallic Systems: Insights from the Anderson Impurity Model, Phys. Rev. B 97, 245136 (2018). 
[133] P. Thunström, O. Gunnarsson, Sergio Ciuchi, and G. Rohringer, Analytical Investigation of Singularities in Two-Particle Irreducible Vertex Functions of the Hubbard Atom, Phys. Rev. B 98, 235107 (2018).

[134] D. Springer, P. Chalupa, S. Ciuchi, G. Sangiovanni, and A. Toschi, Interplay between Local Response and Vertex Divergences in Many-Fermion Systems with On-Site Attraction, Phys. Rev. B 101, 155148 (2020).

[135] M. Reitner, P. Chalupa, L. Del Re, D. Springer, S. Ciuchi, G. Sangiovanni, and A. Toschi, Attractive Effect of a Strong Electronic Repulsion: The Physics of Vertex Divergences, Phys. Rev. Lett. 125, 196403 (2020).

[136] P. Chalupa, T. Schäfer, M. Reitner, D. Springer, S. Andergassen, and A. Toschi, Fingerprints of the Local Moment Formation and its Kondo Screening in the Generalized Susceptibilities of Many-Electron Problems, Phys. Rev. Lett. 126, 056403 (2021).

[137] D. Rohe and C. Honerkamp, Quasi-Particle Functional Renormalisation Group Calculations in the TwoDimensional Half-Filled Hubbard Model at Finite Temperatures, SciPost Phys. 9, 84 (2020).

[138] S. Afchain, J. Magnen, and V. Rivasseau, The Hubbard Model at Half-Filling, Part III: The Lower Bound on the Self-Energy, Ann. Henri Poincaré 6, 449 (2005).

[139] A. Abanov, A. V. Chubukov, and J. Schmalian, QuantumCritical Theory of the Spin-Fermion Model and Its Application to Cuprates: Normal State Analysis, Adv. Phys. 52, 119 (2003).

[140] A. V. Chubukov and D. L. Maslov, First-MatsubaraFrequency Rule in a Fermi Liquid. I. Fermionic SelfEnergy, Phys. Rev. B 86, 155136 (2012).

[141] A. Virosztek and J. Ruvalds, Nested-Fermi-Liquid Theory, Phys. Rev. B 42, 4064 (1990).

[142] A. T. Zheleznyak, V. M. Yakovenko, and Ig. E. Dzyaloshinskii, Parquet Solution for a Flat Fermi Surface, Phys. Rev. B 55, 3200 (1997).

[143] F. V. de Abreu and Douçot B., Nesting Effects on Fermionic Systems, Europhys. Lett. 38, 533 (1997).

[144] D. Zanchi and H. J. Schulz, Weakly Correlated Electrons on a Square Lattice: Renormalization-Group Theory, Phys. Rev. B 61, 13609 (2000).

[145] C. Bertrand, S. Florens, O. Parcollet, and X. Waintal, Reconstructing Nonequilibrium Regimes of Quantum Many-Body Systems from the Analytical Structure of Perturbative Expansions, Phys. Rev. X 9, 041008 (2019).

[146] J. Vučičević and M. Ferrero, Real-Frequency Diagrammatic Monte Carlo at Finite Temperature, Phys. Rev. B 101, 075113 (2020).

[147] A. Taheridehkordi, S. H. Curnoe, and J. P. F. LeBlanc, Algorithmic Approach to Diagrammatic Expansions for Real-Frequency Evaluation of Susceptibility Functions, Phys. Rev. B 102, 045115 (2020).

[148] A. Taheridehkordi, S. H. Curnoe, and J. P. F. LeBlanc, Optimal Grouping of Arbitrary Diagrammatic Expansions via Analytic Pole Structure, Phys. Rev. B 101, 125109 (2020).

[149] M. Kitatani, T. Schäfer, H. Aoki, and K. Held, Why the Critical Temperature of High- $T_{c}$ Cuprate Superconductors
Is So Low: The Importance of the Dynamical Vertex Structure, Phys. Rev. B 99, 041115(R) (2019).

[150] A. Tagliavini, C. Hille, F. Kugler, S. Andergassen, A. Toschi, and C. Honerkamp, Multiloop Functional Renormalization Group for the Two-Dimensional Hubbard Model: Loop Convergence of the Response Functions, SciPost Phys. 6, 009 (2019).

[151] C. Taranto, S. Andergassen, J. Bauer, K. Held, A. Katanin, W. Metzner, G. Rohringer, and A. Toschi, From Infinite to Two Dimensions through the Functional Renormalization Group, Phys. Rev. Lett. 112, 196402 (2014).

[152] D. Vilardi, C. Taranto, and W. Metzner, Antiferromagnetic and $d$-Wave Pairing Correlations in the Strongly Interacting Two-Dimensional Hubbard Model from the Functional Renormalization Group, Phys. Rev. B 99, 104501 (2019).

[153] M. Kitatani, L. Si, O. Janson, R. Arita, Z. Zhong, and K. Held, Nickelate Superconductors-A Renaissance of the One-Band Hubbard Model, npj Quantum Mater. 5, 59 (2020).

[154] A. Galler, P. Thunström, P. Gunacker, J. M. Tomczak, and K. Held, Ab Initio Dynamical Vertex Approximation, Phys. Rev. B 95, 115107 (2017).

[155] H. U. R. Strand, M. Zingl, N. Wentzell, O. Parcollet, and A. Georges, Magnetic Response of $\mathrm{Sr}_{2} \mathrm{RuO}_{4}$ : Quasi-Local Spin Fluctuations Due to Hund's Coupling, Phys. Rev. B 100, 125120 (2019).

[156] A. Tamai, M. Zingl, E. Rozbicki, E. Cappelli, S. Riccò, A. de la Torre, S. M. Walker, F. Y. Bruno, P. D. C. King, W. Meevasana, M. Shi, M. Radović, N. C. Plumb, A. S. Gibbs, A. P. Mackenzie, C. Berthod, H. U. R. Strand, M. Kim, A. Georges, and F. Baumberger, High-Resolution Photoemission on $\mathrm{Sr}_{2} \mathrm{RuO}_{4}$ Reveals Correlation-Enhanced Effective Spin-Orbit Coupling and Dominantly Local Self-Energies, Phys. Rev. X 9, 021048 (2019).

[157] S. Acharya, D. Pashov, C. Weber, H. Park, L. Sponza, and M. Van Schilfgaarde, Evening out the Spin and Charge Parity to Increase $T_{c}$ in $\mathrm{Sr}_{2} \mathrm{RuO}_{4}$, Commun. Phys. 2, 163 (2019).

[158] K. Zantout, S. Backes, and R. Valentí, Effect of Nonlocal Correlations on the Electronic Structure of LiFeAs, Phys. Rev. Lett. 123, 256401 (2019).

[159] O. Gunnarsson, T. Schäfer, J. P. F. LeBlanc, J. Merino, G. Sangiovanni, G. Rohringer, and A. Toschi, Parquet Decomposition Calculations of the Electronic Self-Energy, Phys. Rev. B 93, 245102 (2016).

[160] W. Wu, M. Ferrero, A. Georges, and E. Kozik, Controlling Feynman Diagrammatic Expansions: Physical Nature of the Pseudogap in the Two-Dimensional Hubbard Model, Phys. Rev. B 96, 041105(R) (2017).

[161] G. Rohringer, Spectra of Correlated Many-Electron Systems: From a One- to a Two-Particle Description, J. Electron Spectrosc. Relat. Phenom. 241, 146804 (2018).

[162] B. Arzhang, A. E. Antipov, and J. P. F. LeBlanc, Fluctuation Diagnostics of the Finite-Temperature QuasiAntiferromagnetic Regime of the Two-Dimensional Hubbard Model, Phys. Rev. B 101, 014430 (2020).

[163] O. Gunnarsson, J. Merino, T. Schäfer, G. Sangiovanni, G. Rohringer, and A. Toschi, Complementary Views on 
Electron Spectra: From Fluctuation Diagnostics to RealSpace Correlations, Phys. Rev. B 97, 125134 (2018).

[164] T. Schäfer and A. Toschi, How to Read between the Lines of Electronic Spectra: The Diagnostics of Fluctuations in Strongly Correlated Electron Systems, J. Phys. Condens. Matter (2021) https://doi.org/10.1088/1361-648X/abeb44.

[165] Jülich Supercomputing Centre, JURECA: Modular Supercomputer at Jülich Supercomputing Centre, J. Large-Scale Res. Facil. 4, A132 (2018).

[166] K. Huang, Statistical Mechanics, 2nd ed. (John Wiley \& Sons, New York, 1987).

[167] S. Sachdev, Quantum Phase Transitions (Cambridge University Press, Cambridge, England, 1999).

[168] For obtaining the data on the continuous imaginary frequency axis, in a first step, $\operatorname{Im} \Sigma(\mathbf{k}, \omega)$ has been calculated on the real axis. After retrieving $\operatorname{Re} \Sigma(\mathbf{k}, \omega)$ via the Kramers-Kronig relations, the Cauchy formula has been used for the forward continuation onto the imaginary axis. The calculation on the real axis profits from analytical treatments of the delta function, which reduces the quadrature dimensions.

[169] A. A. Abrikosov, L. P. Gorkov, and I. E. Dzyaloshinski, Methods of Quantum Field Theory in Statistical Physics (Dover, New York, 1975).

[170] R. Rossi, F. Werner, N. Prokof'ev, and B. Svistunov, Shifted-Action Expansion and Applicability of Dressed Diagrammatic Schemes, Phys. Rev. B 93, 161102(R) (2016).

[171] K. Chen and K. Haule, A Combined Variational and Diagrammatic Quantum Monte Carlo Approach to the Many-Electron Problem, Nat. Commun. 10, 3725 (2019).

[172] R. Rossi, F. Šimkovic, and M. Ferrero, Renormalized Perturbation Theory at Large Expansion Orders, Europhys. Lett. 132, 11001 (2020).

[173] F. Šimkovic, R. Rossi, and M. Ferrero, Efficient One-LoopRenormalized Vertex Expansions with Connected Determinant Diagrammatic Monte Carlo, Phys. Rev. B 102, 195122 (2020).

[174] K. Van Houcke, E. Kozik, N. Prokof'ev, and B. Svistunov, Diagrammatic Monte Carlo, Phys. Procedia 6, 95 (2010).

[175] E. Kozik, K. Van Houcke, E. Gull, L. Pollet, N. Prokof'ev, B. Svistunov, and M. Troyer, Diagrammatic Monte Carlo for Correlated Fermions, Europhys. Lett. 90, 10004 (2010).

[176] A. N. Rubtsov, V. V. Savkin, and A. I. Lichtenstein, Continuous-Time Quantum Monte Carlo Method for Fermions, Phys. Rev. B 72, 035122 (2005).

[177] R. Rossi, Direct Sampling of the Self-Energy with Connected Determinant Monte Carlo, arXiv:1802.04743.

[178] K. Van Houcke, F. Werner, E. Kozik, N. Prokof'ev, B. Svistunov, M. J. H. Ku, A. T. Sommer, L. W. Cheuk, A. Schirotzek, and M. W. Zwierlein, Feynman Diagrams versus Fermi-Gas Feynman Emulator, Nat. Phys. 8, 366 (2012).

[179] N. V. Prokof'ev and B. V. Svistunov, Bold Diagrammatic Monte Carlo: A Generic Sign-Problem Tolerant Technique for Polaron Models and Possibly Interacting ManyBody Problems, Phys. Rev. B 77, 125101 (2008).
[180] F. Šimkovic IV, Y. Deng, and E. Kozik, Superfluid Ground-State Phase Diagram of the 2d Hubbard Model in the Emergent BCS Regime,arXiv:1912.13054.

[181] A. Björklund, T. Husfeldt, P. Kaski, and M. Koivisto, Fourier Meets Möbius: Fast Subset Convolution, in Proceedings of the Thirty-Ninth Annual ACM Symposium on Theory of Computing, STOC '07 (Association for Computing Machinery, New York, NY, 2007), p. 67-74.

[182] R. Rossi, N. Prokof'ev, B. Svistunov, K. Van Houcke, and F. Werner, Polynomial Complexity Despite the Fermionic sign, Europhys. Lett. 118, 10004 (2017).

[183] C. Brezinski, Extrapolation Algorithms and Padé Approximations: A Historical Survey, Applied Numerical Mathematics 20, 299 (1996).

[184] P. Gonnet, S. Güttel, and L. N. Trefethen, Robust Padé Approximation via SVD, SIAM Rev. 55, 101 (2013).

[185] C. Lenihan, A. J. Kim, F. Šimkovic IV, and E. Kozik, Entropy in the Non-Fermi-Liquid Regime of the Doped $2 d$ Hubbard Model, arXiv:2001.09948 [Phys. Rev. Lett. (to be published)].

[186] J. E. Hirsch, Discrete Hubbard-Stratonovich Transformation for Fermion Lattice Models, Phys. Rev. B 28, 4059 (1983).

[187] F. F. Assaad and H. G. Evertz, World-Line and Determinantal Quantum Monte Carlo Methods for Spins, Phonons and Electrons, in Computational Many-Particle Physics, Lecture Notes in Physics, edited by H. Fehske, R. Schneider, and A. Weiße (Springer, Berlin, Heidelberg, 2008), Vol. 739, pp. 277-356.

[188] C.-C. Chang, S. Gogolenko, J. Perez, Z. Bai, and R. T. Scalettar, Recent Advances in Determinant Quantum Monte Carlo, Phil. Mag. 95, 1260 (2015).

[189] Y.-Y. He, M. Qin, H. Shi, Z.-Y. Lu, and S. Zhang, FiniteTemperature Auxiliary-Field Quantum Monte Carlo: SelfConsistent Constraint and Systematic Approach to Low Temperatures, Phys. Rev. B 99, 045108 (2019).

[190] Y.-Y. He, H. Shi, and S. Zhang, Reaching the Continuum Limit in Finite-Temperature Ab Initio Field-Theory Computations in Many-Fermion Systems, Phys. Rev. Lett. 123, 136402 (2019).

[191] D. Bohm and D. Pines, A Collective Description of Electron Interactions. I. Magnetic Interactions, Phys. Rev. 82, 625 (1951).

[192] A. N. Rubtsov and A. I. Lichtenstein, Continuous-Time Quantum Monte Carlo Method for Fermions: Beyond Auxiliary Field Framework, JETP Lett. 80, 61 (2004).

[193] E. Gull, A. J. Millis, A. I. Lichtenstein, A. N. Rubtsov, M. Troyer, and P. Werner, Continuous-Time Monte Carlo Methods for Quantum Impurity Models, Rev. Mod. Phys. 83, 349 (2011).

[194] O. Parcollet, M. Ferrero, T. Ayral, H. Hafermann, I. Krivenko, L. Messio, and P. Seth, TRIQS: A Toolbox for Research on Interacting Quantum Systems, Comput. Phys. Commun. 196, 398 (2015).

[195] G. Rohringer, A. Katanin, T. Schäfer, A. Hausoel, K. Held, and A. Toschi, Ladder DГA Code, github.com/ladderDGA (2018).

[196] H. U. R. Strand, N. Wentzell, and O. Parcollet, TPRFTwo-Particle Response Function Tools Based on the TRIQS Library (2019), https://triqs.github.io/tprf/latest/. 
[197] J. P. F. LeBlanc and E. Gull, Equation of State of the Fermionic Two-Dimensional Hubbard Model, Phys. Rev. B 88, 155108 (2013).

[198] E. Gull, P. Werner, O. Parcollet, and M. Troyer, Continuous-Time Auxiliary-Field Monte Carlo for Quantum Impurity Models, Europhys. Lett. 82, 57003 (2008).

[199] E. Gull, P. Staar, S. Fuchs, P. Nukala, M. S. Summers, T. Pruschke, T. C. Schulthess, and T. Maier, Submatrix Updates for the Continuous-Time Auxiliary-Field Algorithm, Phys. Rev. B 83, 075122 (2011).

[200] A. Gaenko, A. E. Antipov, G. Carcassi, T. Chen, X. Chen, Q. Dong, L. Gamper, J. Gukelberger, R. Igarashi, S. Iskakov, M. Könz, J. P. F. LeBlanc, R. Levy, P. N. Ma, J. E. Paki, H. Shinaoka, S. Todo, M. Troyer, and E. Gull, Updated Core Libraries of the ALPS Project, Comput. Phys. Commun. 213, 235 (2017).

[201] M. Wallerberger, S. Iskakov, A. Gaenko, J. Kleinhenz, I. Krivenko, R. Levy, J. Li, H. Shinaoka, S. Todo, T. Chen, X. Chen, J. P. F. LeBlanc, J. E. Paki, H. Terletska, M. Troyer, and E. Gull, Updated Core Libraries of the ALPS Project, arXiv:1811.08331.

[202] S. Sakai, G. Sangiovanni, M. Civelli, Y. Motome, K. Held, and M. Imada, Cluster-Size Dependence in Cellular Dynamical Mean-Field Theory, Phys. Rev. B 85, 035102 (2012).

[203] T. D. Stanescu and G. Kotliar, Fermi Arcs and Hidden Zeros of the Green Function in the Pseudogap State, Phys. Rev. B 74, 125110 (2006).

[204] S. Sakai, Y. Motome, and M. Imada, Evolution of Electronic Structure of Doped Mott Insulators: Reconstruction of Poles and Zeros of Green's Function, Phys. Rev. Lett. 102, 056404 (2009).

[205] P. Staar, T. A. Maier, M. S. Summers, G. Fourestey, Ra. Solca, and T. C. Schulthess, Taking a Quantum Leap in Time to Solution for Simulations of High- $T_{c}$ Superconductors, in Proceedings of the International Conference on High Performance Computing, Networking, Storage and Analysis (ACM, New York, 2013), pp. 1:1-1:11.

[206] S. Sakai, M. Civelli, and M. Imada, Hidden Fermionic Excitation Boosting High-Temperature Superconductivity in Cuprates, Phys. Rev. Lett. 116, 057003 (2016).

[207] S. Verret, J. Roy, A. Foley, M. Charlebois, D. Sénéchal, and A.-M. S. Tremblay, Intrinsic Cluster-Shaped Density Waves in Cellular Dynamical Mean-Field Theory, Phys. Rev. B 100, 224520 (2019).

[208] A. Valli, G. Sangiovanni, O. Gunnarsson, A. Toschi, and K. Held, Dynamical Vertex Approximation for Nanoscopic Systems, Phys. Rev. Lett. 104, 246402 (2010).

[209] A. Valli, T. Schäfer, P. Thunström, G. Rohringer, S. Andergassen, G. Sangiovanni, K. Held, and A. Toschi, Dynamical Vertex Approximation in Its Parquet Implementation: Application to Hubbard Nanorings, Phys. Rev. B 91, 115115 (2015).

[210] A. Kauch, P. Pudleiner, K. Astleithner, P. Thunström, T. Ribic, and K. Held, Generic Optical Excitations of Correlated Systems: $\pi$-Tons, Phys. Rev. Lett. 124, 047401 (2020).

[211] A. Kauch, F. Hörbinger, G. Li, and K. Held, Interplay between Magnetic and Superconducting Fluctuations in the Doped 2d Hubbard Model, arXiv:1901.09743.
[212] T. Schäfer, A. Toschi, and Jan M. Tomczak, Separability of Dynamical and Nonlocal Correlations in Three Dimensions, Phys. Rev. B 91, 121107(R) (2015).

[213] P. Pudleiner, T. Schäfer, D. Rost, G. Li, K. Held, and N. Blümer, Momentum Structure of the Self-Energy and Its Parametrization for the Two-Dimensional Hubbard Model, Phys. Rev. B 93, 195134 (2016).

[214] T. Schäfer, A. A. Katanin, K. Held, and A. Toschi, Interplay of Correlations and Kohn Anomalies in Three Dimensions: Quantum Criticality with a Twist, Phys. Rev. Lett. 119, 046402 (2017).

[215] T. Schäfer, A. A. Katanin, M. Kitatani, A. Toschi, and K. Held, Quantum Criticality in the Two-Dimensional Periodic Anderson Model, Phys. Rev. Lett. 122, 227201 (2019).

[216] L. Del Re, M. Capone, and A. Toschi, Dynamical Vertex Approximation for the Attractive Hubbard Model, Phys. Rev. B 99, 045137 (2019).

[217] B. Klebel-Knobloch, T. Schäfer, A. Toschi, and J. M. Tomczak, Anisotropy of Electronic Correlations: On the Applicability of Local Theories to Layered Materials, Phys. Rev. B 103, 045121 (2021).

[218] T. Schäfer, Classical and Quantum Phase Transitions in Strongly Correlated Electron Systems, Ph.D. Thesis, TU Wien, 2016, https://repositum.tuwien.at/handle/20.500 $.12708 / 3282$.

[219] G. Rohringer, New Routes towards a Theoretical Treatment of Nonlocal Electronic Correlations, Ph.D. Thesis, Vienna University of Technology, 2013, https://repositum .tuwien.at/handle/20.500.12708/6957.

[220] T. Ayral and O. Parcollet, Mott Physics and Spin Fluctuations: A Unified Framework, Phys. Rev. B 92, 115109 (2015).

[221] T. Ayral and O. Parcollet, Mott Physics and Spin Fluctuations: A Functional Viewpoint, Phys. Rev. B 93, 235124 (2016).

[222] T. Ayral, J. Vučičević, and O. Parcollet, Fierz Convergence Criterion: A Controlled Approach to Strongly Interacting Systems with Small Embedded Clusters, Phys. Rev. Lett. 119, 166401 (2017).

[223] J. Vučičević, T. Ayral, and O. Parcollet, TRILEX and $G W+$ EDMFT Approach to d-Wave Superconductivity in the Hubbard Model, Phys. Rev. B 96, 104504 (2017).

[224] E. A. Stepanov, A. Huber, E. G. C. P. van Loon, A. I. Lichtenstein, and M. I. Katsnelson, From Local to Nonlocal Correlations: The Dual Boson Perspective, Phys. Rev. B 94, 205110 (2016).

[225] E. A. Stepanov, V. Harkov, and A. I. Lichtenstein, Consistent Partial Bosonization of the Extended Hubbard Model, Phys. Rev. B 100, 205115 (2019).

[226] F. Krien, Efficient Evaluation of the Polarization Function in Dynamical Mean-Field Theory, Phys. Rev. B 99, 235106 (2019).

[227] F. Krien, E. G. C. P. van Loon, H. Hafermann, J. Otsuki, M. I. Katsnelson, and A. I. Lichtenstein, Conservation in Two-Particle Self-Consistent Extensions of Dynamical Mean-Field Theory, Phys. Rev. B 96, 075155 (2017).

[228] A. N. Rubtsov, M. I. Katsnelson, and A. I. Lichtenstein, Dual Fermion Approach to Nonlocal Correlations in the Hubbard Model, Phys. Rev. B 77, 033101 (2008). 
[229] A. N. Rubtsov, M. I. Katsnelson, A. I. Lichtenstein, and A. Georges, Dual Fermion Approach to the Two-Dimensional Hubbard Model: Antiferromagnetic Fluctuations and Fermi Arcs, Phys. Rev. B 79, 045133 (2009).

[230] H. Hafermann, G. Li, A. N. Rubtsov, M. I. Katsnelson, A. I. Lichtenstein, and H. Monien, Efficient Perturbation Theory for Quantum Lattice Models, Phys. Rev. Lett. 102, 206401 (2009).

[231] A. E. Antipov, J. P. F. LeBlanc, and E. Gull, Opendf-An Implementation of the Dual Fermion Method for Strongly Correlated Systems, Phys. Procedia 68, 43 (2015).

[232] S. K. Sarker, A New Functional Integral Formalism for Strongly Correlated Fermi Systems," J. Phys. C 21, L667 (1988).

[233] S. Pairault, D. Sénéchal, and A.-M. S. Tremblay, StrongCoupling Perturbation Theory of the Hubbard Model, Eur. Phys. J. B 16, 85 (2000).

[234] C. Bourbonnais, Fluctuations Quantiques dans les Syst'emes a Basse Dimensionnalité: Théorie et Applications aux Conducteurs Organiques, Ph.D. thesis, Université de Sherbrooke, 1985.

[235] G. Rohringer, A. Valli, and A. Toschi, Local Electronic Correlation at the Two-Particle Level, Phys. Rev. B 86, 125114 (2012).

[236] T. Ribic, P. Gunacker, S. Iskakov, M. Wallerberger, G. Rohringer, A. N. Rubtsov, E. Gull, and K. Held, Role of Three-Particle Vertex within Dual Fermion Calculations, Phys. Rev. B 96, 235127 (2017).

[237] S. Iskakov, A. E. Antipov, and E. Gull, Diagrammatic Monte Carlo for Dual Fermions, Phys. Rev. B 94, 035102 (2016).

[238] J. Gukelberger, E. Kozik, and H. Hafermann, Diagrammatic Monte Carlo Approach for Diagrammatic Extensions of Dynamical Mean-Field Theory: Convergence Analysis of the Dual Fermion Technique, Phys. Rev. B 96, 035152 (2017).

[239] J. P. F. LeBlanc, S. Li, X. Chen, R. Levy, A. E. Antipov, A. J. Millis, and E. Gull, Magnetic Susceptibility and Simulated Neutron Signal in the Two-Dimensional Hubbard Model, Phys. Rev. B 100, 075123 (2019).

[240] E. G. C. P. van Loon, H. Hafermann, and M. I. Katsnelson, Precursors of the Insulating State in the Square Lattice Hubbard Model, Phys. Rev. B 97, 085125 (2018).

[241] G. V. Astretsov, G. Rohringer, and A. N. Rubtsov, Dual Parquet Scheme for the Two-Dimensional Hubbard Model: Modeling Low-Energy Physics of High- $T_{c}$ Cuprates with High Momentum Resolution, Phys. Rev. B 101, 075109 (2020).

[242] F. Krien, A. Valli, P. Chalupa, M. Capone, A. I. Lichtenstein, and A. Toschi, Boson-Exchange Parquet Solver for Dual Fermions, Phys. Rev. B 102, 195131 (2020).

[243] K. Astleithner, A. Kauch, T. Ribic, and K. Held, Parquet Dual Fermion Approach for the Falicov-Kimball Model, Phys. Rev. B 101, 165101 (2020).

[244] A. N. Rubtsov, M. I. Katsnelson, and A. I. Lichtenstein, Dual Boson Approach to Collective Excitations in Correlated Fermionic Systems, Ann. Phys. (N.Y.) 327, 1320 (2012).

[245] E. G. C. P. van Loon, A. I. Lichtenstein, M. I. Katsnelson, O. Parcollet, and H. Hafermann, Beyond Extended
Dynamical Mean-Field Theory: Dual Boson Approach to the Two-Dimensional Extended Hubbard Model, Phys. Rev. B 90, 235135 (2014).

[246] E. A. Stepanov, E. G. C. P. van Loon, A. A. Katanin, A. I. Lichtenstein, M. I. Katsnelson, and A. N. Rubtsov, SelfConsistent Dual Boson Approach to Single-Particle and Collective Excitations in Correlated Systems, Phys. Rev. B 93, 045107 (2016).

[247] P. Sun and G. Kotliar, Extended Dynamical Mean-Field Theory and GW Method, Phys. Rev. B 66, 085120 (2002).

[248] P. Sun and G. Kotliar, Many-Body Approximation Scheme beyond GW, Phys. Rev. Lett. 92, 196402 (2004).

[249] L. Peters, E. G. C. P. van Loon, A. N. Rubtsov, A. I. Lichtenstein, M. I. Katsnelson, and E. A. Stepanov, Dual Boson Approach with Instantaneous Interaction, Phys. Rev. B 100, 165128 (2019).

[250] E. G. C. P. van Loon, H. Hafermann, A. I. Lichtenstein, A. N. Rubtsov, and M. I. Katsnelson, Plasmons in Strongly Correlated Systems: Spectral Weight Transfer and Renormalized Dispersion, Phys. Rev. Lett. 113, 246407 (2014).

[251] E. A. Stepanov, A. Huber, A. I. Lichtenstein, and M. I. Katsnelson, Effective Ising Model for Correlated Systems with Charge Ordering, Phys. Rev. B 99, 115124 (2019).

[252] E. A. Stepanov, S. Brener, F. Krien, M. Harland, A. I. Lichtenstein, and M. I. Katsnelson, Effective Heisenberg Model and Exchange Interaction for Strongly Correlated Systems, Phys. Rev. Lett. 121, 037204 (2018).

[253] E. A. Stepanov, L. Peters, I. S. Krivenko, A. I. Lichtenstein, M. I. Katsnelson, and A. N. Rubtsov, Quantum Spin Fluctuations and Evolution of Electronic Structure in Cuprates, npj Quantum Mater. 3, 54 (2018).

[254] H. Hafermann, E. G. C. P. van Loon, M. I. Katsnelson, A. I. Lichtenstein, and O. Parcollet, Collective Charge Excitations of Strongly Correlated Electrons, Vertex Corrections, and Gauge Invariance, Phys. Rev. B 90, 235105 (2014).

[255] H. Hafermann, P. Werner, and E. Gull, Efficient Implementation of the Continuous-Time Hybridization Expansion Quantum Impurity Solver, Comput. Phys. Commun. 184, 1280 (2013).

[256] H. Hafermann, Self-Energy and Vertex Functions from Hybridization-Expansion Continuous-Time Quantum Monte Carlo for Impurity Models with Retarded Interaction, Phys. Rev. B 89, 235128 (2014).

[257] B. Bauer et al., The ALPS Project Release 2.0: Open Source Software for Strongly Correlated Systems, J. Stat. Mech. (2011) P05001.

[258] Y. M. Vilk, L. Chen, and A.-M. S. Tremblay, Theory of Spin and Charge Fluctuations in the Hubbard Model, Phys. Rev. B 49, 13267 (1994).

[259] K. S. Singwi and M. P. Tosi, Solid State Physics, edited by H. Ehrenreich, F. Seitz, and D. Turnbull (Academic, New York, 1981).

[260] S. Ichimaru, Strongly Coupled Plasmas: High-Density Classical Plasmas and Degenerate Electron Liquids, Rev. Mod. Phys. 54, 1017 (1982).

[261] M. R. Hedayati and G. Vignale, Ground-State Energy of the One- and Two-Dimensional Hubbard Model Calcu- 
lated by the Method of Singwi, Tosi, Land, and Sjölander, Phys. Rev. B 40, 9044 (1989).

[262] S. Allen, A.-M. S. Tremblay, and Y. M. Vilk, Conserving Approximations vs. Two-Particle Self-Consistent Approach, in Theoretical Methods for Strongly Correlated Electrons, edited by D. Sénéchal, C. Bourbonnais, and A.-M. S. Tremblay (Springer-Verlag, New York, Berlin, Heidelberg, 2004), Chap. 8, pp. 341-356.

[263] L. P. Kadanoff and P. C. Martin, Theory of Many-Particle Systems. II. Superconductivity, Phys. Rev. 124, 670 (1961).

[264] Q. Chen, J. Stajic, S. Tan, and K. Levin, BCS-BEC Crossover: From High Temperature Superconductors to Ultracold Superfluids, Phys. Rep. 412, 1 (2005).

[265] R. Boyack, Q. Chen, A. A. Varlamov, and K. Levin, Cuprate Diamagnetism in the Presence of a Pseudogap: Beyond the Standard Fluctuation Formalism, Phys. Rev. B 97, 064503 (2018).

[266] M. Salmhofer, Renormalization-An Introduction, edited by R. Balian, W. Beiglböck, H. Grosse, E. H. Lieb, N. Reshetikhin, H. Spohn, and W. Thirring (Springer-Verlag, Berlin, Heidelberg, 1999).

[267] J. Berges, N. Tetradis, and C. Wetterich, Non-perturbative Renormalization Flow in Quantum Field Theory and Statistical Physics, Phys. Rep. 363, 223 (2002).

[268] P. Kopietz, L. Bartosch, and F. Schüt, Introduction to the Functional Renormalization Group, Lecture Notes in Physics Vol. 798 (Springer-Verlag, Berlin, Heidelberg, 2010).

[269] J. J. Deisz, D. W. Hess, and J. W. Serene, Incipient Antiferromagnetism and Low-Energy Excitations in the Half-Filled Two-Dimensional Hubbard Model, Phys. Rev. Lett. 76, 1312 (1996).

[270] A. A. Katanin and A. P. Kampf, Quasiparticle Anisotropy and Pseudogap Formation from the Weak-Coupling Renormalization Group Point of View, Phys. Rev. Lett. 93, 106406 (2004).

[271] D. Rohe and W. Metzner, Pseudogap at Hot Spots in the Two-Dimensional Hubbard Model at Weak Coupling, Phys. Rev. B 71, 115116 (2005).

[272] A. Kampf and A. A. Katanin, Quasiparticle Anisotropy and Pseudogap Formation: A Weak-Coupling Renormalization-Group Analysis, J. Phys. Chem. Solids 67, 146 (2006).

[273] Strongly correlated parameter regimes are beyond the oneloop flow but might become accessible by exploiting the DMFT as a starting point for the fRG flow [151,152].

[274] C. Husemann and M. Salmhofer, Efficient Parametrization of the Vertex Function, $\Omega$ Scheme, and the $t, t^{\prime}$ Hubbard Model at van Hove Filling, Phys. Rev. B 79, 195125 (2009).
[275] X. Wang, M. J. Han, L. de' Medici, H. Park, C. A. Marianetti, and A. J. Millis, Covalency, Double-Counting, and the Metal-Insulator Phase Diagram in Transition Metal Oxides, Phys. Rev. B 86, 195136 (2012).

[276] J. Lichtenstein, D. S. de la Peña, D. Rohe, E. Di Napoli, C. Honerkamp, and S. A. Maier, High-Performance Functional Renormalization Group Calculations for Interacting Fermions, Comput. Phys. Commun. 213, 100 (2017).

[277] D. Vilardi, C. Taranto, and W. Metzner, Nonseparable Frequency Dependence of the Two-Particle Vertex in Interacting Fermion Systems, Phys. Rev. B 96, 235110 (2017).

[278] A. A. Katanin, Fulfillment of Ward Identities in the Functional Renormalization Group Approach, Phys. Rev. B 70, 115109 (2004).

[279] C. De Dominicis and P. C. Martin, Stationary Entropy Principle and Renormalization in Normal and Superfluid Systems. I. Algebraic Formulation, J. Math. Phys. (N.Y.) 5, 14 (1964).

[280] A. N. Vasil'ev, A. K. Kazanskii, and Yu. M. Pis'mak, Diagrammatic Analysis of the Fourth Legendre Transform, Theor. Math. Phys. 20, 754 (1974).

[281] S. X. Yang, H. Fotso, J. Liu, T. A. Maier, K. Tomko, E. F. D’Azevedo, R. T. Scalettar, T. Pruschke, and M. Jarrell, Parquet Approximation for the $4 \times 4$ Hubbard Cluster, Phys. Rev. E 80, 046706 (2009).

[282] K.-M. Tam, H. Fotso, S.-X. Yang, T.-W. Lee, J. Moreno, J. Ramanujam, and M. Jarrell, Solving the Parquet Equations for the Hubbard Model beyond Weak Coupling, Phys. Rev. E 87, 013311 (2013).

[283] G. Li, A. Kauch, P. Pudleiner, and K. Held, The Victory Project v1.0: An Efficient Parquet Equations Solver, Comput. Phys. Commun. 241, 146 (2019).

[284] P. Pudleiner, P. Thunström, A. Valli, A. Kauch, G. Li, and K. Held, Parquet Approximation for Molecules: Spectrum and Optical Conductivity of the Pariser-Parr-Pople Model, Phys. Rev. B 99, 125111 (2019).

[285] P. Pudleiner, A. Kauch, K. Held, and G. Li, Competition between Antiferromagnetic and Charge Density Wave Fluctuations in the Extended Hubbard Model, Phys. Rev. B 100, 075108 (2019).

[286] G. Li, N. Wentzell, P. Pudleiner, P. Thunström, and K. Held, Efficient Implementation of the Parquet Equations: Role of the Reducible Vertex Function and Its Kernel Approximation, Phys. Rev. B 93, 165103 (2016).

[287] J. Kaufmann, C. Eckhardt, M. Pickem, M. Kitatani, A. Kauch, and K. Held, Self-Consistent Ladder Dynamical Vertex Approximation, Phys. Rev. B 103, 035120 (2021).

[288] F. Krien, A. Kauch, and K. Held, Tiling with Triangles: Parquet and $G W \gamma$ Methods Unified, Phys. Rev. Research 3, 013149 (2021). 